\title{
Radical Germylzincation of Aryl- and Alkyl-Substituted Internal Alkynes
}

Karen de la Vega-Hernández, Fabrice Chemla, Franck Ferreira, Olivier Jackowski, and Alejandro Perez-Luna*

Sorbonne Université, CNRS, Institut Parisien de Chimie Moléculaire, F-75005 Paris, France E-mail: alejandro.perez_luna@sorbonne-universite.fr

\section{Supporting Information}

\section{Table of Contents}

I Additional Data and Discussion $\quad$ S2

Table S1. Control Experiments in the Absence of $\mathrm{Et}_{2} \mathrm{Zn}$ : Direct Radical S2 Hydrogermylation of Selected Propargylic and Homopropargylic alcohols

II Experimental Details $\quad$ S2

II.1 General Information $\quad$ S2

II.2 Experimental Procedures and Compound Characterization Data S3

II.2.1 General Procedure for the Germylzincation of Aryl- and Alkyl-Substituted S3 Internal Alkynes (GP I)

II.2.2 General Procedure for the Domino Alkyne Germylzincation-Cu(I)- S3 Mediated Electrophilic Trapping of Aryl- and Alkyl-Substituted Internal Alkynes (GP II)

II.2.3 Compound Characterization Data $\quad$ S4

II.3 NMR Spectra for New Compounds $\quad$ S21

II.4 X-Ray Crystal Structures of compounds 6, E-8a, Z-15, E-17 S82 


\section{Additional Data and Discussion}

Table S1. Control Experiments in the Absence of $\mathrm{Et}_{2} \mathrm{Zn}$ : Direct Radical Hydrogermylation of Selected Propargylic and Homopropargylic alcohols.

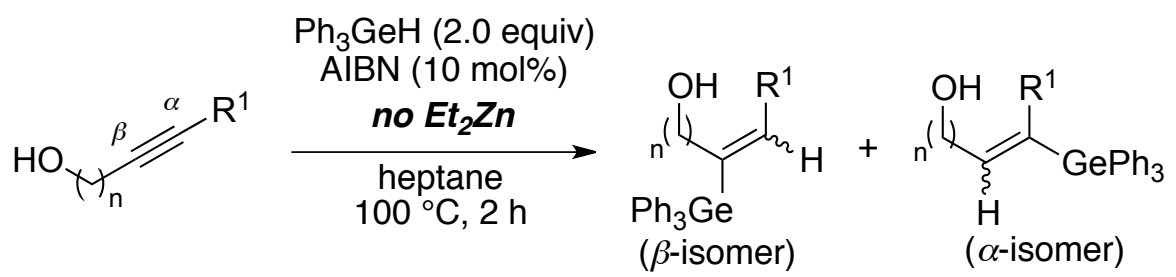

$9 a, 9 c, 10$

13a, 13c, 14

\begin{tabular}{|c|c|c|c|c|c|c|c|c|}
\hline Entry & Substrate & $\mathbf{n}$ & $\mathbf{R}^{\mathbf{1}}$ & Product & $\boldsymbol{\beta} / \boldsymbol{\alpha}^{\mathbf{a}}$ & ${\text { d.r. } \boldsymbol{\alpha}(\boldsymbol{E} / \mathbf{Z})^{\mathbf{a}}}$ & ${\text { d.r. } \boldsymbol{\beta}(\boldsymbol{E} / \mathbf{Z})^{\mathbf{a}}}$ & Yield (\%) $^{\mathbf{b}}$ \\
\hline 1 & $\mathbf{9 a}$ & 1 & $\mathrm{Ph}$ & $\mathbf{1 3 a}$ & $>98: 2$ & - & $86: 14$ & 84 \\
\hline 2 & $\mathbf{9 c}$ & 1 & $n \mathrm{Hept}$ & $\mathbf{1 3 c}$ & $76: 24$ & $61: 39$ & $61: 39$ & 92 \\
\hline 3 & $\mathbf{1 0}$ & 2 & $n \mathrm{Hex}$ & $\mathbf{1 4}$ & $57: 43$ & $66: 34$ & $67: 33$ & 84 \\
\hline
\end{tabular}

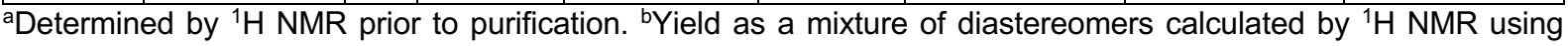
butadiene sulfone as internal standard.

These control experiments evidence an O-directing effect in the addition of the triphenylgermyl radical across the $\mathrm{C}-\mathrm{C}$ triple bond of propargyloxy dialkylacetylenes (compare entries 2 and 3). With substrate $9 a$ (entry 1), as for other (alkyl, aryl)-substituted alkynes, the addition of the triphenylgermyl radical is fully $\beta$-regioselective, what can be ascribed to stabilization by the adjacent phenyl group of the vinyl radical intermediate.

\section{Experimental Details}

\section{II.1 General Information}

Experiments involving organometallic compounds were carried out in dried glassware under a positive pressure of dry argon. THF was distilled to remove stabilizers and dried with a Solvent Purification System. $\mathrm{Et}_{2} \mathrm{Zn}$ (Aldrich), triphenylgermanium hydride (Aldrich), heptane (Aldrich), and all other reagents were of commercial quality and were used without purification. Flash column chromatography was performed using the indicated solvents on silica gel 60 (40-63 $\mu \mathrm{m}, 230-400$ mesh, ASTM) by Merck. ${ }^{1} \mathrm{H}$ NMR and ${ }^{13} \mathrm{C}$ NMR spectra were recorded with a Bruker AV 300 or 400 spectrometers. Chemical shifts are reported in parts per million (ppm) downfield from tetramethylsilane and are referenced to the residual solvent resonance as the internal standard $\left(\mathrm{CHCl}_{3}: \delta=7.26 \mathrm{ppm}\right.$ for ${ }^{1} \mathrm{H}$ NMR and $\mathrm{CDCl}_{3}: \delta=77.16 \mathrm{ppm}$ for $\left.{ }^{13} \mathrm{C} \mathrm{NMR}\right)$. Data are reported as follows: chemical shift, multiplicity (br $s=$ broad singlet, $s=$ singlet, $d=$ doublet, $\mathrm{t}=$ triplet, $\mathrm{q}=$ quartet, quint $=$ quintuplet, hept = heptaplet, $\mathrm{m}=$ multiplet), coupling constant $(\mathrm{Hz})$ and integration. Infrared $(\mathrm{IR})$ spectra were recorded with a Bruker Tensor 27 
ATR diamond spectrophotometer and are reported in wavenumbers $\left(\mathrm{cm}^{-1}\right)$. High-resolution mass spectra (ESI-MS and APCI-MS) were acquired using an LTQ-Orbitrap XL from Thermo Scientific (Thermo Fisher Scientific, Courtaboeuf, France) operated in positive ionization mode. Melting points $(\mathrm{mp})$ were determined with Stuart Scientific SMP3 melting point apparatus and are not corrected. Substrates $\mathbf{1 a - c}, \mathbf{4 a - b}, 7 \mathbf{a}-\mathbf{b}, \mathbf{9 a}-\mathbf{c}, 9 \mathbf{e}$ and 10 are commercially available and were used as received. Substrates $1 \mathbf{d},{ }^{1} \mathbf{1} \mathrm{e}^{2}$ and $\mathbf{9} \mathbf{d}^{3}$ were prepared according to literature procedures.

\section{II.2 Experimental Procedures and Compound Characterization Data}

\section{II.2.1 General Procedure for the Germylzincation of Aryl- and Alkyl-Substituted} Internal Alkynes (GP I)

In a dry tube under argon atmosphere, the appropriate alkyne ( $0.25 \mathrm{mmol}, 1.0$ equiv), $\mathrm{Ph}_{3} \mathrm{GeH}$ (153 mg, $0.50 \mathrm{mmol}, 2.0$ equiv), and AIBN (10-100 mol\%) were dissolved in the appropriate solvent $(0.64 \mathrm{~mL})$. The tube was then sealed with a cap with septum, and $\mathrm{Et}_{2} \mathrm{Zn}(1.0 \mathrm{M}$ in hexane, $0.75 \mathrm{~mL}, 0.75 \mathrm{mmol}, 3.0$ equiv) was added at $\mathrm{rt}$. The reaction mixture was either placed in an oil bath pre-heated at the suitable temperature or placed in the microwave reactor (Biotage, Model: INITIATOR ROBOT EIGHT 355380, 30310-38V), and stirred for the given reaction time. After cooling the reaction mixture to rt, aq $\mathrm{NH}_{4} \mathrm{Cl} / \mathrm{NH}_{3}(2: 1)$ and $\mathrm{CH}_{2} \mathrm{Cl}_{2}$ were added. The layers were separated, the aqueous layer was extracted with $\mathrm{CH}_{2} \mathrm{Cl}_{2}(\mathrm{x} 3)$, and the combined organic layers were washed with brine (x2), dried over $\mathrm{Na}_{2} \mathrm{SO}_{4}$, filtered, and concentrated under reduce pressure to afford the crude product.

\section{II.2.2 General Procedure for the Domino Alkyne Germylzincation-Cu(I)-Mediated}

Electrophilic Trapping of Aryl- and Alkyl-Substituted Internal Alkynes (GP II)

In a dry tube under argon atmosphere, the appropriate alkyne ( $0.25 \mathrm{mmol}, 1.0$ equiv), $\mathrm{Ph}_{3} \mathrm{GeH}$ (153 mg, $0.50 \mathrm{mmol}, 2.0$ equiv), and AIBN (10-100 mol\%) were dissolved in the appropriate solvent $(0.64 \mathrm{~mL})$. The tube was then sealed with a cap with septum, and $\mathrm{Et}_{2} \mathrm{Zn}(1.0 \mathrm{M}$ in hexane, $0.75 \mathrm{~mL}, 0.75 \mathrm{mmol}, 3.0$ equiv) was added at rt. The reaction mixture was either placed in an oil bath pre-heated at the suitable temperature or placed in the microwave reactor (Biotage, Model: INITIATOR ROBOT EIGHT 355380, 30310-38V), and stirred for the given reaction time. The reaction was then progressively cooled to $-30^{\circ} \mathrm{C}$. Next, $\mathrm{CuCN} \cdot 2 \mathrm{LiCl}(1.0 \mathrm{M}$ in THF, $0.75 \mathrm{mmol}, 3.0$ equiv) followed by the appropriate electrophile ( $1.75 \mathrm{mmol}, 7.0$ equiv) were added at this temperature and the reaction mixture was allowed to warm slowly to $\mathrm{rt}$ overnight under stirring. Then, aq $\mathrm{NH}_{4} \mathrm{Cl} / \mathrm{NH}_{3}(2: 1)$ and $\mathrm{CH}_{2} \mathrm{Cl}_{2}$ were added and after $1 \mathrm{~h}$ stirring

\footnotetext{
${ }^{1}$ Mouriès, V.; Waschbüsch, R.; Carran, J.; Savignac, P. Synthesis 1998, 271-274.

${ }^{2}$ Cai, R.; Lu, M.; Aguilera, E. Y.; Xi, Y.; Akhmedov, N. G.; Petersen, J. L.; Chen, H.; Shi, X. Angew. Chemie Int. Ed. 2015, 54, 8772-8776.

${ }^{3}$ Trost, B. M.; Ball, Z. T. J. Am. Chem. Soc. 2005, 127, 17644-17655.
} 
the layers were separated. The aqueous layer was extracted with $\mathrm{CH}_{2} \mathrm{Cl}_{2}(\mathrm{x} 3)$, and the combined organic layers were washed with brine (x2), dried over $\mathrm{Na}_{2} \mathrm{SO}_{4}$, filtered, and concentrated under reduce pressure to afford the crude product.

\section{II.2.3 Compound Characterization Data}

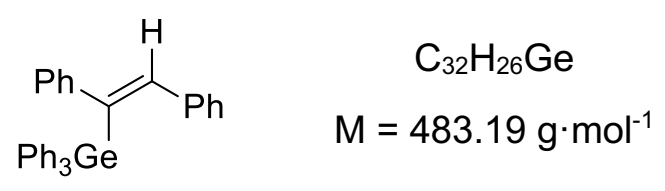

3a

(Z)-(1,2-diphenylvinyl)triphenylgermane (3a): The preparation according to GP I of compound 3a (Table 1, entry 2) using diphenylacetylene $1 \mathrm{a}$ ( $45 \mathrm{mg}, 0.25 \mathrm{mmol}, 1.0$ equiv) as substrate, $\mathrm{Et}_{2} \mathrm{Zn}\left(1.0 \mathrm{M}\right.$ in hexane, $0.75 \mathrm{~mL}, 0.75 \mathrm{mmol}, 3.0$ equiv), $\mathrm{Ph}_{3} \mathrm{GeH}$ (153 mg, 0.50 $\mathrm{mmol}, 2.0$ equiv), AIBN (10 mg, $0.06 \mathrm{mmol}, 25 \mathrm{~mol} \%)$ in dry THF (0.64 mL) and a reaction time of $1 \mathrm{~h}$ at $80^{\circ} \mathrm{C}$ was previously reported in a preliminary survey. ${ }^{[4]} \mathrm{NMR}$ characterization data is recalled hereafter for convenience.

${ }^{1} \mathrm{H}$ NMR $\left(400 \mathrm{MHz}, \mathrm{CDCl}_{3}\right)$ : ( $\mathrm{Z}$ isomer) $\delta 7.54(\mathrm{~s}, 1 \mathrm{H}), 7.31-7.27(\mathrm{~m}, 6 \mathrm{H}), 7.16-7.03(\mathrm{~m}, 13 \mathrm{H})$, 7.01-6.95 (m, 3H), 6.82-6.78 (m, 1H), 6.76-6.72 (m, 2H).

${ }^{13} \mathrm{C}\left\{{ }^{1} \mathrm{H}\right\}$ NMR $\left(100 \mathrm{MHz}, \mathrm{CDCl}_{3}\right):(Z$ isomer $) \delta 146.3,146.1,141.3,137.6,137.2,135.3,128.9$, 128.6, 128.3, 128.0, 127.8, 127.5, 127.3, 126.1.<smiles>CCCCc1ccc(/C=C(\OC(=O)c2ccccc2)c2ccc(CCCC)cc2)cc1</smiles>

3b

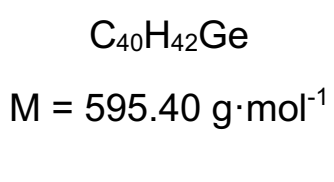

$\mathrm{M}=595.40 \mathrm{~g} \cdot \mathrm{mol}^{-1}$

(Z)-(1,2-bis(4-butylphenyl)vinyl)triphenylgermane (3b): Prepared according to GP I using 1,2-bis(4-butylphenyl)ethyne $1 \mathrm{~b}$ (73 $\mathrm{mg}, 0.25 \mathrm{mmol}, 1.0$ equiv) as substrate, $\mathrm{Et}_{2} \mathrm{Zn}$ (1.0 M in hexane, $0.75 \mathrm{~mL}, 0.75 \mathrm{mmol}, 3.0$ equiv), $\mathrm{Ph}_{3} \mathrm{GeH}$ (153 mg, $0.50 \mathrm{mmol}, 2.0$ equiv), AIBN (10 $\mathrm{mg}, 0.06 \mathrm{mmol}, 25 \mathrm{~mol} \%)$ in dry THF $(0.64 \mathrm{~mL})$ and a reaction time of $1 \mathrm{~h}$ at $80^{\circ} \mathrm{C}$. Purification of the crude product $(Z / E=90: 10)$ by flash chromatography on silica gel (eluent pentane/toluene $=90: 10)$ afforded analytically pure $3 \mathbf{b}(101 \mathrm{mg}, 68 \%, Z / E=90: 10)$ as a white solid; mp $75-78^{\circ} \mathrm{C}$.

\footnotetext{
${ }^{4}$ de la Vega-Hernández, K. ; Romain, E.; Coffinet, A.; Bijouard, K.; Gontard, G.; Chemla, F.; Ferreira, F.; Jackowski, O.; Perez-Luna. A. Radical Germylzincation of $\alpha$-Heteroatom-Substituted Alkynes. J. Am. Chem. Soc. 2018, 140, 17632-17642.
} 
${ }^{1} \mathrm{H}$ NMR $\left(400 \mathrm{MHz}, \mathrm{CDCl}_{3}\right):(\mathrm{Z}$ isomer) $\delta 7.70(\mathrm{~s}, 1 \mathrm{H}), 7.49-7.45(\mathrm{~m}, 6 \mathrm{H}), 7.33-7.23(\mathrm{~m}, 9 \mathrm{H})$, 7.16-7.11 (m, 4H), 6.99-6.97 (m, 2H), 6.74-6.72 (m, 2H), $2.58(\mathrm{t}, J=7.6 \mathrm{~Hz}, 2 \mathrm{H}), 2.45(\mathrm{t}, J=$ $7.6 \mathrm{~Hz}, 2 \mathrm{H}$ ), 1.59 (quint, $J=7.6 \mathrm{~Hz}, 2 \mathrm{H}$ ), 1.49 (quint, $J=7.6 \mathrm{~Hz}, 2 \mathrm{H}), 1.41-1.26(\mathrm{~m}, 4 \mathrm{H}), 1.00$ $0.95(\mathrm{~m}, 6 \mathrm{H})$.

${ }^{13} \mathrm{C}\left\{{ }^{1} \mathrm{H}\right\}$ NMR $\left(100 \mathrm{MHz}, \mathrm{CDCl}_{3}\right):(Z$ isomer $) \delta 145.9,143.5,141.9,140.5,140.2,137.5,135.7$, 135.3, 128.8, 128.5, 128.2, 127.9, 127.87, 127.5, 35.32, 35.3, 33.7, 33.5, 22.3, 22.2, 14.1.

IR (neat): $v\left(\mathrm{~cm}^{-1}\right)$ 2955, 2927, 2856, 1430, 1088, 907, 732, 697.

HRMS (APCl): $m / z$ calculated for $\left[\mathrm{C}_{40} \mathrm{H}_{42} \mathrm{Ge}+\mathrm{H}\right]^{+}$597.2571; found 597.2557.

The Z configuration was assigned by analogy with Z-3a.

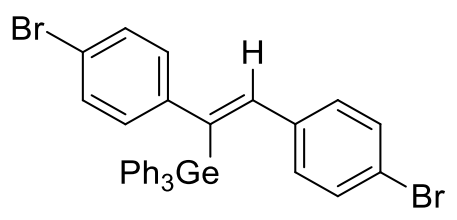

$\mathrm{C}_{32} \mathrm{H}_{24} \mathrm{Br}_{2} \mathrm{Ge}$
$M=640.98 \mathrm{~g} \cdot \mathrm{mol}^{-1}$

$3 c$

(Z)-(1,2-bis(4-bromophenyl)vinyl)triphenylgermane (3c): Prepared according to GP I using 1,2-bis(4-bromophenyl)ethyne 1c (84 mg, $0.25 \mathrm{mmol}, 1.0$ equiv) as substrate, $\mathrm{Et}_{2} \mathrm{Zn}(1.0 \mathrm{M}$ in hexane, $0.75 \mathrm{~mL}, 0.75 \mathrm{mmol}, 3.0$ equiv), $\mathrm{Ph}_{3} \mathrm{GeH}$ (153 mg, $0.50 \mathrm{mmol}, 2.0$ equiv), AIBN (10 $\mathrm{mg}, 0.06 \mathrm{mmol}, 25 \mathrm{~mol} \%)$ in dry THF $(0.64 \mathrm{~mL})$ and a reaction time of $1 \mathrm{~h}$ at $80^{\circ} \mathrm{C}$. Purification of the crude product $(Z / E=87: 13)$ by flash chromatography on silica gel (eluent pentane/toluene $=90: 10)$ afforded analytically pure $3 \mathrm{c}(105 \mathrm{mg}, 66 \%, Z / E=87: 13)$ as a white solid; mp 205-208 ${ }^{\circ} \mathrm{C}$.

${ }^{1} \mathrm{H}$ NMR $\left(400 \mathrm{MHz}, \mathrm{CDCl}_{3}\right):(\mathrm{Z}$ isomer) $\delta 7.42(\mathrm{~s}, 1 \mathrm{H}), 7.28-7.25(\mathrm{~m}, 6 \mathrm{H}), 7.22-7.18(\mathrm{~m}, 2 \mathrm{H})$, 7.14-7.09 (m, 9H), 6.90-6.81 (m, 6H).

${ }^{13} \mathrm{C}\left\{{ }^{1} \mathrm{H}\right\}$ NMR $\left(100 \mathrm{MHz}, \mathrm{CDCl}_{3}\right):(Z$ isomer $) \delta 145.3,144.7,141.8,136.4,135.1,130.9,130.6$, $130.3,129.9,128.9,128.2,128.1,121.7,120.3$.

IR (neat): $v\left(\mathrm{~cm}^{-1}\right)$ 2997, 2956, 1484, 1431, 1089, 826, 733, 697.

HRMS (APCI): $m / z$ calculated for $\left[\mathrm{C}_{32} \mathrm{H}_{24} \mathrm{Br}_{2} \mathrm{Ge}+\mathrm{H}\right]^{+} 640.9529$; found 640.9520 .

The Z configuration was assigned by analogy with Z-3a. 
<smiles>COc1ccc(C=C(c2ccc(C(F)(F)F)cc2)C(F)(F)F)cc1</smiles>

$\beta-3 d$<smiles>COc1ccc(/C(=C/c2ccc(C(F)(F)F)cc2)C(=O)c2ccccc2)cc1</smiles>

$\alpha-3 d$

(2-(4-methoxyphenyl)-1-(4-(trifluoromethyl)phenyl)vinyl) triphenylgermane $(\beta-3 d)$ and (1-(4-methoxyphenyl)-2-(4-(trifluoromethyl) phenyl)vinyl)triphenylgermane ( $\alpha$-3d): Prepared according to GP I using 1-methoxy-4-((4-(trifluoromethyl)phenyl)ethynyl)benzene 1d (69 mg, $0.25 \mathrm{mmol}, 1.0$ equiv) as substrate, $\mathrm{Et}_{2} \mathrm{Zn}(1.0 \mathrm{M}$ in hexane, $0.75 \mathrm{~mL}, 0.75 \mathrm{mmol}, 3.0$ equiv), $\mathrm{Ph}_{3} \mathrm{GeH}$ (153 mg, $0.50 \mathrm{mmol}, 2.0$ equiv), AIBN (10 mg, $0.06 \mathrm{mmol}, 25 \mathrm{~mol} \%$ ) in dry THF $(0.64 \mathrm{~mL})$ and a reaction time of $1 \mathrm{~h}$ at $80{ }^{\circ} \mathrm{C}$. Purification of the crude product by flash chromatography on silica gel (eluent pentane/toluene/Et $\left.{ }_{2} \mathrm{O}=85: 10: 5\right)$ afforded $\mathbf{3 d}(111 \mathrm{mg}$, $76 \%$ ) as mixture of four isomers in 39:38:13:10 ratio.

${ }^{1} \mathrm{H}$ NMR $\left(400 \mathrm{MHz}, \mathrm{CDCl}_{3}\right):(\beta$-3d (Z-isomer) $+\alpha$-3d (Z-isomer)) $\delta 7.52(\mathrm{~s}, 1 \mathrm{H}), 7.46(\mathrm{~s}, 1 \mathrm{H})$, 7.35-7.00 (m, 40H), 6.61-6.53 (m, 4H), 6.30-6.27 (m, 2H), $3.60(\mathrm{~s}, 3 \mathrm{H}), 3.49(\mathrm{~s}, 3 \mathrm{H})$.

${ }^{13} \mathbf{C}\left\{{ }^{1} \mathrm{H}\right\}$ NMR $\left(100 \mathrm{MHz}, \mathrm{CDCl}_{3}\right):(\boldsymbol{\beta}$-3d $(Z$-isomer $)+\alpha$-3d $(Z$-isomer $)) \delta 159.2,158.5,150.18$, $150.16,146.7,144.0,143.7,141.4,138.1,137.9,136.9,136.6,135.2,130.4,129.3,128.9$, 128.8, 128.7, 128.4, 128.18, 128.15, $128.0\left(q,{ }^{2} J_{C-F}=32.2 \mathrm{~Hz}\right), 124.7\left(q,{ }^{3} J_{C-F}=3.7 \mathrm{~Hz}\right), 124.5$ $\left(\mathrm{q},{ }^{1} J_{C-F}=271.9 \mathrm{~Hz}\right), 124.3\left(\mathrm{q},{ }^{3} J_{C-F}=3.8 \mathrm{~Hz}\right), 124.2\left(\mathrm{q},{ }^{1} J_{C-F}=272.0 \mathrm{~Hz}\right), 113.5,113.1,55.3$, 55.2. (one $C$ is not visible)

${ }^{19} \mathrm{~F}\left\{{ }^{1} \mathrm{H}\right\}$ NMR $\left(376 \mathrm{MHz}, \mathrm{CDCl}_{3}\right):(\boldsymbol{\beta}$-3d $(Z$-isomer $)+\alpha-3 \mathbf{d}(Z$-isomer $)) \delta-62.7,-62.2$.

HRMS (APCl): $m / z$ calculated for $\left[\mathrm{C}_{34} \mathrm{H}_{27} \mathrm{~F}_{3} \mathrm{GeO}+\mathrm{H}\right]^{+}$583.1299; found 583.1297.

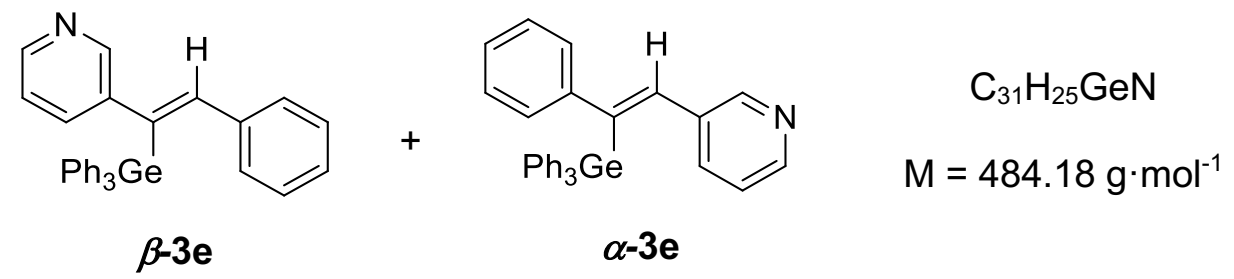

(Z)-3-(2-phenyl-1-(triphenylgermyl)vinyl)pyridine ( $\beta$-3e) and (Z)-3-(2-phenyl-1-(triphenylgermyl)vinyl)pyridine ( $\alpha$-3e): Prepared according to GP I using 3-(phenylethynyl)pyridine 1 e (45 mg, $0.25 \mathrm{mmol}, 1.0$ equiv) as substrate, $\mathrm{Et}_{2} \mathrm{Zn}(1.0 \mathrm{M}$ in hexane, $0.75 \mathrm{~mL}, 0.75 \mathrm{mmol}, 3.0$ equiv), $\mathrm{Ph}_{3} \mathrm{GeH}$ (153 mg, $0.50 \mathrm{mmol}, 2.0$ equiv), AIBN (10 mg, $0.06 \mathrm{mmol}, 25 \mathrm{~mol} \%$ ) in dry THF $(0.64 \mathrm{~mL})$ and a reaction time of $1 \mathrm{~h}$ at $80^{\circ} \mathrm{C}$. Purification of the crude product (mixture of regioisomers in ratio: $\beta-3 e / \alpha-3 e=65(Z / E>98: 2): 35(Z / E>98: 2)$ ) by flash chromatography on silica gel (eluent pentane/toluene/ $\mathrm{Et}_{2} \mathrm{O}=50: 30: 20$ ) afforded two fractions containing $\beta$-3e (70 mg, $57 \%$, ZIE > 98:2) and $\alpha-3 e(36 \mathrm{mg}, 31 \%, Z / E>98: 2)$. 
$\beta$-3e:

White solid; $\mathrm{mp} 169-172^{\circ} \mathrm{C}$.

${ }^{1} \mathrm{H}$ NMR $\left(400 \mathrm{MHz}, \mathrm{CDCl}_{3}\right): \delta 8.27(\mathrm{dd}, J=4.8 \mathrm{~Hz}, J=2.0 \mathrm{~Hz}, 1 \mathrm{H}), 8.13(\mathrm{~d}, J=2.0 \mathrm{~Hz}, 1 \mathrm{H})$, 7.38-7.35 (m, 6H), 7.30-7.24 (m, 9H), 7.11 (dt, J = 7.8 Hz, J=2.0 Hz, 1H), 7.03-7.01 (m, 3H), $6.95(\mathrm{~s}, 1 \mathrm{H}), 6.94-6.92(\mathrm{~m}, 1 \mathrm{H}), 6.90-6.85(\mathrm{~m}, 2 \mathrm{H})$.

${ }^{13} \mathrm{C}\left\{{ }^{1} \mathrm{H}\right\}$ NMR $\left(100 \mathrm{MHz}, \mathrm{CDCl}_{3}\right): \delta 149.1,147.3,142.7,138.6,138.1,136.5,135.7,135.6$, 135.2, 129.6, 129.4, 128.5, 128.3, 127.8, 123.3.

IR (neat): $v\left(\mathrm{~cm}^{-1}\right)$ 3068, 3049, 1484, 1431, 1090, 1025, 734, 697.

HRMS (APCI) : m/z calculated for $\left[\mathrm{C}_{31} \mathrm{H}_{25} \mathrm{GeN}+\mathrm{H}\right]^{+}$486.1272; found 486.1273. $\alpha-3 e:$

${ }^{1} \mathrm{H}$ NMR $\left(400 \mathrm{MHz}, \mathrm{CDCl}_{3}\right): \delta 8.40-8.20(\mathrm{~m}, 2 \mathrm{H}), 7.45-7.31(\mathrm{~m}, 15 \mathrm{H}), 7.20-7.13(\mathrm{~m}, 4 \mathrm{H}), 7.00-$ $6.90(\mathrm{~m}, 3 \mathrm{H}), 6.87(\mathrm{~s}, 1 \mathrm{H})$.

${ }^{13} \mathrm{C}\left\{{ }^{1} \mathrm{H}\right\}$ NMR $\left(100 \mathrm{MHz}, \mathrm{CDCl}_{3}\right): \delta 151.0,148.1,146.2,141.5,136.9,136.0,135.6,135.5$, $135.2,129.3,128.9,128.4,127.8,126.6,122.9$.

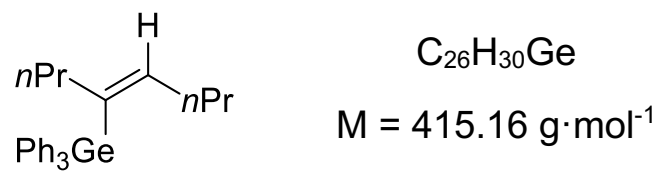

$5 a$

(Z)-oct-4-en-4-yltriphenylgermane (5a): Prepared according to GP I using 4-octyne 4a (28 $\mathrm{mg}, 0.25 \mathrm{mmol}, 1.0$ equiv) as substrate, $\mathrm{Et}_{2} \mathrm{Zn}(1.0 \mathrm{M}$ in hexane, $0.75 \mathrm{~mL}, 0.75 \mathrm{mmol}, 3.0$ equiv), $\mathrm{Ph}_{3} \mathrm{GeH}$ (153 mg, $0.50 \mathrm{mmol}, 2.0$ equiv), AIBN (41 mg, $0.25 \mathrm{mmol}, 100 \mathrm{~mol} \%$ ) in dry THF $(0.64 \mathrm{~mL})$. The reaction mixture was then heated by microwave irradiation at $80{ }^{\circ} \mathrm{C}(50$ $\mathrm{W}$ ) for $1 \mathrm{~h}$ (normal absorption level). Purification of the crude product $(Z / E=91: 9)$ by flash chromatography on silica gel (eluent pentane/toluene $=90: 10$ ) afforded analytically pure $\mathbf{5 a}$ (60 mg, 58\%, Z/E = 95:5) as a colorless liquid.

${ }^{1} \mathrm{H}$ NMR (400 MHz, $\left.\mathrm{CDCl}_{3}\right)$ : ( $\mathrm{Z}$ isomer) $\delta 7.57-7.55(\mathrm{~m}, 6 \mathrm{H}), 7.38-7.34(\mathrm{~m}, 9 \mathrm{H}), 6.27$ (tt, $\mathrm{J}=$ $7.4 \mathrm{~Hz}, J=1.2 \mathrm{~Hz}, 1 \mathrm{H}), 2.13-2.09(\mathrm{~m}, 2 \mathrm{H}), 1.84(\mathrm{q}, J=7.4 \mathrm{~Hz}, 2 \mathrm{H}), 1.28-1.12(\mathrm{~m}, 4 \mathrm{H}), 0.69$ (t, $J=7.4 \mathrm{~Hz}, 3 \mathrm{H}), 0.58(\mathrm{t}, J=7.4 \mathrm{~Hz}, 3 \mathrm{H})$.

${ }^{13} \mathrm{C}\left\{{ }^{1} \mathrm{H}\right\}$ NMR $\left(100 \mathrm{MHz}, \mathrm{CDCl}_{3}\right):(\mathrm{Z}$ isomer $) \delta 143.4,137.9,136.0,135.3,128.8,128.2,41.1$, 35.6, 23.7, 22.8, 13.74, 13.7.

IR (neat): $v\left(\mathrm{~cm}^{-1}\right)$ 2997, 2958, 2870, 1460, 1431, 1089, 735, 699.

HRMS (ESI): $\mathrm{m} / \mathrm{z}$ calculated for $\left[\mathrm{C}_{26} \mathrm{H}_{30} \mathrm{Ge}+\mathrm{Na}\right]^{+} 439.1452$; found 439.1451 . 


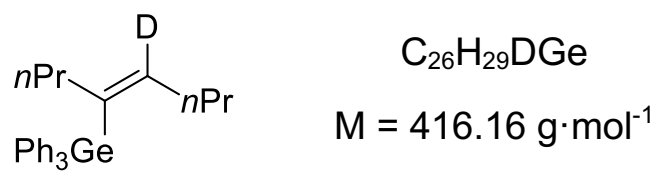

$\left[{ }^{2} \mathrm{H}\right]-5 \mathrm{a}$

$\left[{ }^{2} \mathrm{H}\right]-5 \mathrm{a}$ : Prepared according to GP I using 4-octyne 4a (28 mg, $0.25 \mathrm{mmol}, 1.0$ equiv) as substrate, $\mathrm{Et}_{2} \mathrm{Zn}\left(1.0 \mathrm{M}\right.$ in hexane, $0.75 \mathrm{~mL}, 0.75 \mathrm{mmol}, 3.0$ equiv), $\mathrm{Ph}_{3} \mathrm{GeH}$ (153 mg, 0.50 mmol, 2.0 equiv), AIBN (41 mg, $0.25 \mathrm{mmol}, 100 \mathrm{~mol} \%$ ) in dry THF (0.64 mL). The reaction mixture was then heated in the microwave at $80{ }^{\circ} \mathrm{C}(50 \mathrm{~W})$ for $1 \mathrm{~h}$ (normal absorption level). After cooling the reaction mixture to $\mathrm{rt}$, a solution of $\mathrm{ND}_{4} \mathrm{Cl}(300 \mathrm{mg})$ in $\mathrm{D}_{2} \mathrm{O}(2 \mathrm{~mL})$ was added and the mixture stirred for $1 \mathrm{~h}$ prior to the addition of aq $\mathrm{NH}_{4} \mathrm{Cl} / \mathrm{NH}_{3}(2: 1)$ and $\mathrm{CH}_{2} \mathrm{Cl}_{2}$. The usual work-up was then carried out. Purification of the crude product $(Z / E=89: 11)$ by flash chromatography on silica gel (eluent pentane/toluene $=90: 10$ ) afforded analytically pure $\left[{ }^{2} \mathrm{H}\right]$ 5a (64 mg, 62\%, ZIE = 95:5) as a colorless liquid.

${ }^{1} \mathrm{H}$ NMR (400 MHz, $\left.\mathrm{CDCl}_{3}\right)$ : ( $\mathrm{Z}$ isomer) $\delta ~ 7.57-7.54(\mathrm{~m}, 6 \mathrm{H}), 7.40-7.33(\mathrm{~m}, 9 \mathrm{H}), 2.12-2.08(\mathrm{~m}$, $2 \mathrm{H}), 1.83(\mathrm{t}, J=7.4 \mathrm{~Hz}, 2 \mathrm{H}), 1.28-1.11(\mathrm{~m}, 4 \mathrm{H}), 0.69(\mathrm{t}, J=7.4 \mathrm{~Hz}, 3 \mathrm{H}), 0.58(\mathrm{t}, J=7.4 \mathrm{~Hz}$, $3 \mathrm{H})$.

${ }^{13} \mathrm{C}\left\{{ }^{1} \mathrm{H}\right\}$ NMR $\left(100 \mathrm{MHz}, \mathrm{CDCl}_{3}\right)$ : ( $\mathrm{Z}$ isomer) $\delta 137.9,135.9,135.3,128.8,128.2,41.0,35.5$, $23.7,22.8,13.74,13.7$. One $C$ is not observed.

HRMS (ESI): $m / z$ calculated for $\left[{ }_{26} \mathrm{H}_{29} \mathrm{DGe}+\mathrm{Na}\right]^{+} 440.1514$; found 440.1514 .<smiles>CCOC(=O)/C=C(/C)C(C)C</smiles>

$\beta-5 b$<smiles>C/C=C(\c1ccccc1)C(C)C</smiles>

$\alpha-5 b$

(4-methylpent-2-en-2-yl)triphenylgermane $(\beta-5 b)$ and (4-methylpent-2-en-3-yl) triphenylgermane ( $\alpha$-5b): Prepared according to GP I using 4-methylpent-2-yne $4 \mathbf{b}$ (21 mg, 0.25 mmol, 1.0 equiv) as substrate, $\mathrm{Et}_{2} \mathrm{Zn}\left(1.0 \mathrm{M}\right.$ in hexane, $0.75 \mathrm{~mL}, 0.75 \mathrm{mmol}, 3.0$ equiv), $\mathrm{Ph}_{3} \mathrm{GeH}$ (153 $\mathrm{mg}, 0.50 \mathrm{mmol}, 2.0$ equiv), AIBN (4 mg, $0.025 \mathrm{mmol}, 10 \mathrm{~mol} \%)$ in heptane $(0.64 \mathrm{~mL})$. The reaction mixture was then heated in the microwave at $100{ }^{\circ} \mathrm{C}(160 \mathrm{~W})$ for $1 \mathrm{~h}$ (normal absorption level). Purification of the crude product (mixture of regioisomers in ratio: $\beta-5 b / \alpha-5 b$ $=44(E / Z=9: 91): 56(E / Z=37: 63))$ by flash chromatography on silica gel (eluent pentane) afforded a mixture of $\beta-5 b$ and $\alpha-5 b$ (54 mg, $56 \%, \beta-5 b / \alpha-5 b$ 48(E/Z = 8:92):52(E/Z = 33:67)), contaminated with $27 \%$ unreacted $\mathrm{Ph}_{3} \mathrm{GeH}$ which was suitable for NMR characterization. 
${ }^{1} \mathrm{H}$ NMR $\left(400 \mathrm{MHz}, \mathrm{CDCl}_{3}\right): \delta 7.65-7.58(\mathrm{~m}, 6 \mathrm{H}), 7.45-7.38(\mathrm{~m}, 9 \mathrm{H}), 6.49(\mathrm{qd}, J=6.9 \mathrm{~Hz}, \mathrm{~J}=$

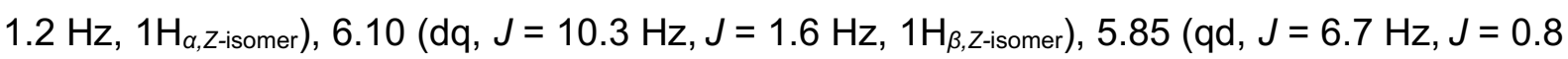
$\mathrm{Hz}, 1 \mathrm{H}_{\alpha, E \text {-isomer }}$ ), 5.71 (dq, $J=8.9 \mathrm{~Hz}, J=1.7 \mathrm{~Hz}, 1 \mathrm{H}_{\beta, E \text {-isomer }}$ ), 3.17-3.07 (m, 1 $\left.\mathrm{H}_{\alpha, E \text {-isomer }}\right), 2.90$ (dhept, $J=8.8 \mathrm{~Hz}, J=6.6 \mathrm{~Hz}, 1 \mathrm{H}_{\beta, E \text {-isomer }}$ ), $2.49-2.39$ ( $\mathrm{m}, 1 \mathrm{H}_{\alpha, Z \text {-isomer }}$ ), 2.21 (dhept, $J=10.3 \mathrm{~Hz}$, $\left.J=6.5 \mathrm{~Hz}, 1 \mathrm{H}_{\beta, Z \text {-isomer }}\right), 1.95\left(\mathrm{~d}, J=1.8 \mathrm{~Hz}, 3 \mathrm{H}_{\beta, E \text {-isomer }}\right), 1.90-1.88\left(\mathrm{~m}, 3 \mathrm{H}_{\beta, Z \text {-isomer }}+3 \mathrm{H}_{\alpha, E \text {-isomer }}\right)$, 1.56 (dd, $\left.J=6.9 \mathrm{~Hz}, J=0.9 \mathrm{~Hz}, 3 \mathrm{H}_{\alpha, Z \text {-isomer }}\right), 1.05-1.00\left(\mathrm{~m}, 6 \mathrm{H}_{\beta, E \text {-isomer }}+6 \mathrm{H}_{\alpha, E \text {-isomer }}+6 \mathrm{H}_{\alpha, Z \text {-isomer }}\right)$, $0.75\left(\mathrm{~d}, J=6.5 \mathrm{~Hz}, 6 \mathrm{H}_{\beta, Z \text {-isomer }}\right.$ ).

${ }^{13} \mathrm{C}\left\{{ }^{1} \mathrm{H}\right\}$ NMR $\left(100 \mathrm{MHz}, \mathrm{CDCl}_{3}\right): \delta 150.9,144.7,143.8,138.4,138.1,137.6,136.9,135.7$, 135.66, 135.5, 135.31, 135.3, 133.3, 129.3, 128.9, 128.8, 128.7, 128.5, 128.22, 128.2, 128.1, 128.06, 34.2, 31.9, 30.9, 26.6, 23.1, 22.63, 22.6, 19.8, 15.1.

HRMS (APCl): $\mathrm{m} / \mathrm{z}$ calculated for $\left[\mathrm{C}_{24} \mathrm{H}_{26} \mathrm{Ge}+\mathrm{H}\right]^{+}-\mathrm{C}_{6} \mathrm{H}_{6}=\left[\mathrm{C}_{18} \mathrm{H}_{21} \mathrm{Ge}\right]^{+}$311.0850; found 311.0850 .

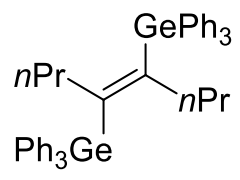

6
$\mathrm{C}_{44} \mathrm{H}_{44} \mathrm{Ge}_{2}$
$\mathrm{M}=718.10 \mathrm{~g} \cdot \mathrm{mol}^{-1}$

(E)-oct-4-ene-4,5-diylbis(triphenylgermane) (6): The product was isolated (57 $\mathrm{mg}, 32 \%, E / Z$ $>$ 98:2) as byproduct in the reaction according to GP I using 4-octyne $4 a$ ( $28 \mathrm{mg}, 0.25 \mathrm{mmol}$, 1.0 equiv) as substrate, $\mathrm{Et}_{2} \mathrm{Zn}\left(1.0 \mathrm{M}\right.$ in hexane, $0.75 \mathrm{~mL}, 0.75 \mathrm{mmol}, 3.0$ equiv), $\mathrm{Ph}_{3} \mathrm{GeH}$ (153 $\mathrm{mg}, 0.50 \mathrm{mmol}, 2.0$ equiv) and AIBN (20 mg, $0.125 \mathrm{mmol}, 50 \mathrm{~mol} \%)$ in heptane $(0.64 \mathrm{~mL})$. The reaction mixture was heated in the microwave at $100{ }^{\circ} \mathrm{C}(160 \mathrm{~W})$ for $1 \mathrm{~h}$ (normal absorption level).

${ }^{1} \mathrm{H}$ NMR $\left(400 \mathrm{MHz}, \mathrm{CDCl}_{3}\right): \delta$ 7.86-7.84 (m, 12H), 7.60-7.58 (m, 18H), 2.38-2.34 (m, 4H), $0.91-0.82(\mathrm{~m}, 4 \mathrm{H}), 0.00(\mathrm{t}, J=7.2 \mathrm{~Hz}, 6 \mathrm{H})$.

${ }^{13} \mathrm{C}\left\{{ }^{1} \mathrm{H}\right\}$ NMR $\left(100 \mathrm{MHz}, \mathrm{CDCl}_{3}\right): \delta 150.1,138.4,135.4,128.8,128.2,42.1,23.2,13.3$.

IR (neat): $v\left(\mathrm{~cm}^{-1}\right)$ 3068, 3049, 2958, 2928, 1431, 1088, 734, 699.

The structure of 6 was established by X-ray crystallographic analysis (CCDC 2077757). 
<smiles>C/C([GeH2])=C\c1ccccc1</smiles>

$$
\begin{gathered}
\mathrm{C}_{27} \mathrm{H}_{24} \mathrm{Ge} \\
\mathrm{M}= \\
421.12 \mathrm{~g} \cdot \mathrm{mol}^{-1}
\end{gathered}
$$

\begin{abstract}
$E-8 a$
(E)-triphenyl(1-phenylprop-1-en-2-yl)germane (E-8a): Prepared according to GP I using 1phenyl-1-propyne 7a (29 mg, $0.25 \mathrm{mmol}, 1.0$ equiv) as substrate, $\mathrm{Et}_{2} \mathrm{Zn}$ (1.0 $\mathrm{M}$ in hexane, 0.75 $\mathrm{mL}, 0.75 \mathrm{mmol}, 3.0$ equiv), $\mathrm{Ph}_{3} \mathrm{GeH}$ (153 mg, $0.50 \mathrm{mmol}, 2.0$ equiv), AIBN (4 mg, $0.03 \mathrm{mmol}$, $10 \mathrm{~mol} \%)$ in heptane $(0.64 \mathrm{~mL})$ and a reaction time of $2 \mathrm{~h}$ at $100{ }^{\circ} \mathrm{C}$. Purification of the crude product $(\beta / \alpha=97(E / Z=95: 5): 3)$ by flash chromatography on silica gel (eluent pentane/toluene $=90: 10)$ afforded analytically pure $E-8 \mathbf{a}(98 \mathrm{mg}, 93 \%, E / Z=96: 4)$ as a white solid; $\mathrm{mp} 165-$ $168^{\circ} \mathrm{C}$.
\end{abstract}

${ }^{1} \mathrm{H}$ NMR $\left(400 \mathrm{MHz}, \mathrm{CDCl}_{3}\right)$ : ( $E$ isomer) $\delta 7.63-7.61(\mathrm{~m}, 6 \mathrm{H}), 7.45-7.41(\mathrm{~m}, 9 \mathrm{H}), 7.38-7.34(\mathrm{~m}$, $4 \mathrm{H}), 7.28-7.23(\mathrm{~m}, 1 \mathrm{H}), 6.88(\mathrm{q}, J=1.8 \mathrm{~Hz}, 1 \mathrm{H}), 2.22(\mathrm{~d}, J=1.8 \mathrm{~Hz}, 3 \mathrm{H})$.

${ }^{13} \mathrm{C}\left\{{ }^{1} \mathrm{H}\right\} \operatorname{NMR}\left(100 \mathrm{MHz}, \mathrm{CDCl}_{3}\right):(E$ isomer $) \delta 141.0,138.0,136.7,136.2,135.6,129.18$, $129.17,128.4,128.2,126.9,19.1$.

IR (neat): $v\left(\mathrm{~cm}^{-1}\right)$ 3067, 3049, 3022, 1485, 1430, 1090, 735, 698.

The structure of E-8a was determined by X-ray crystallographic analysis (CCDC 2077610).<smiles>[2H]/C(=C(\C)c1ccccc1)c1ccccc1</smiles>

$\mathrm{C}_{27} \mathrm{H}_{23} \mathrm{DGe}$
$\mathrm{M}=422.13 \mathrm{~g} \cdot \mathrm{mol}^{-1}$

\title{
$E-\left[{ }^{2} \mathrm{H}\right]-8 \mathbf{a}$
}

\section{$E-\left[{ }^{2} \mathrm{H}\right]-8 \mathrm{a}:$}

Prepared according to GP I using 1-phenyl-1-propyne 7a (29 mg, $0.25 \mathrm{mmol}, 1.0$ equiv) as substrate, $\mathrm{Et}_{2} \mathrm{Zn}\left(1.0 \mathrm{M}\right.$ in hexane, $0.75 \mathrm{~mL}, 0.75 \mathrm{mmol}, 3.0$ equiv), $\mathrm{Ph}_{3} \mathrm{GeH}$ (153 mg, 0.50 $\mathrm{mmol}, 2.0$ equiv), AIBN (4 mg, $0.03 \mathrm{mmol}, 10 \mathrm{~mol} \%$ ) in heptane $(0.64 \mathrm{~mL})$ and a reaction time of $2 \mathrm{~h}$ at $100{ }^{\circ} \mathrm{C}$. After cooling the reaction mixture to rt, a solution of $\mathrm{ND}_{4} \mathrm{Cl}(300 \mathrm{mg})$ in $\mathrm{D}_{2} \mathrm{O}$ $(2 \mathrm{~mL})$ was added, followed after $10 \mathrm{~min}$ by THF $(2 \mathrm{~mL})$, and the mixture was stirred for $1 \mathrm{~h}$ prior to the addition of aq $\mathrm{NH}_{4} \mathrm{Cl} / \mathrm{NH}_{3}(2: 1)$ and $\mathrm{CH}_{2} \mathrm{Cl}_{2}$. The usual work-up was then carried out. Purification by flash chromatography on silica gel (eluent pentane/toluene $=90: 10$ ) afforded analytically pure $E-\left[{ }^{2} \mathrm{H}\right]-8 \mathrm{a}(104 \mathrm{mg}, 99 \%, E / Z=97: 3)$ as a white solid.

${ }^{1} \mathrm{H}$ NMR (400 MHz, CDCl $)$ : ( $E$ isomer) $\delta 7.64-7.60(\mathrm{~m}, 6 \mathrm{H}), 7.45-7.41(\mathrm{~m}, 9 \mathrm{H}), 7.38-7.34(\mathrm{~m}$, $4 \mathrm{H}), 7.28-7.24(\mathrm{~m}, 1 \mathrm{H}), 2.22(\mathrm{~s}, 3 \mathrm{H})$. 
${ }^{13} \mathrm{C}\left\{{ }^{1} \mathrm{H}\right\}$ NMR $\left(100 \mathrm{MHz}, \mathrm{CDCl}_{3}\right):(E$ isomer $) \delta 140.6(\mathrm{t}, J=23.6 \mathrm{~Hz}), 137.9,136.5,136.2,135.6$, 129.2, 129.17, 128.4, 128.2, 126.9, 19.1.

HRMS (ESI): $m / z$ calculated for $\left[\mathrm{C}_{27} \mathrm{H}_{23} \mathrm{DGe}+\mathrm{K}\right]^{+} 462.0784$; found 462.0786 .<smiles>CCC(O)=Cc1ccccc1</smiles>

$8 b$

\author{
$\mathrm{C}_{28} \mathrm{H}_{26} \mathrm{Ge}$ \\ $\mathrm{M}=435.15 \mathrm{~g} \cdot \mathrm{mol}^{-1}$
}

(E)-triphenyl(1-phenylbut-1-en-2-yl)germane (8b): GP I was adjusted to operate at a 1.0 mmol scale. In a dry tube under argon atmosphere, 1-phenyl-1-butyne $7 \mathrm{~b}$ (130 mg, $1.00 \mathrm{mmol}$, 1.0 equiv), $\mathrm{Ph}_{3} \mathrm{GeH}$ (305 mg, $2.00 \mathrm{mmol}, 2.0$ equiv), and AIBN (41 mg, $0.25 \mathrm{mmol}, 25 \mathrm{~mol} \%$ ) were dissolved in heptane $(5.6 \mathrm{~mL})$. The tube was then sealed with a cap with septum, and $\mathrm{Et}_{2} \mathrm{Zn}(1.0 \mathrm{M}$ in hexane, $3.00 \mathrm{~mL}, 3.00 \mathrm{mmol}, 3.0$ equiv) was added at rt. The reaction mixture was placed in an oil bath pre-heated at $100{ }^{\circ} \mathrm{C}$ and stirred for $5 \mathrm{~h}$. After cooling the reaction mixture to rt, aq $\mathrm{NH}_{4} \mathrm{Cl} / \mathrm{NH}_{3}(2: 1)$ and $\mathrm{CH}_{2} \mathrm{Cl}_{2}$ were added. The layers were separated, the aqueous layer was extracted with $\mathrm{CH}_{2} \mathrm{Cl}_{2}(\mathrm{x} 3)$, and the combined organic layers were washed with brine (x2), dried over $\mathrm{Na}_{2} \mathrm{SO}_{4}$, filtered, and concentrated under reduce pressure. Purification of the crude product $(\beta / \alpha=95(E / Z=95: 5): 5)$ by flash chromatography on silica gel (eluent pentane/toluene $=90: 10$ ) afforded analytically pure $8 \mathbf{b}(409 \mathrm{mg}, 94 \%, E / Z=95: 5)$ as a white solid; $\mathrm{mp} 112-115^{\circ} \mathrm{C}$.

${ }^{1} \mathrm{H}$ NMR (400 MHz, CDCl $)$ : ( $E$ isomer) $\delta 7.52-7.49(\mathrm{~m}, 6 \mathrm{H}), 7.31-7.26(\mathrm{~m}, 9 \mathrm{H}), 7.25-7.20(\mathrm{~m}$, 4H), 7.16-7.12 (m, 1H), $6.73(\mathrm{~s}, 1 \mathrm{H}), 2.47(\mathrm{qd}, J=7.5 \mathrm{~Hz}, J=1.1 \mathrm{~Hz}, 2 \mathrm{H}), 0.84(\mathrm{t}, J=7.5 \mathrm{~Hz}$, $3 \mathrm{H})$.

${ }^{13} \mathrm{C}\left\{{ }^{1} \mathrm{H}\right\}$ NMR $\left(100 \mathrm{MHz}, \mathrm{CDCl}_{3}\right):(E$ isomer $) \delta 143.5,140.9,138.0,136.8,135.6,129.1,128.8$, 128.4, 128.3, 127.0, 25.1, 14.8.

IR (neat): $v\left(\mathrm{~cm}^{-1}\right)$ 3067, 3049, 2963, 1485, 1430, 1089, 734, 697.

HRMS (ESI): $m / z$ calculated for $\left[\mathrm{C}_{28} \mathrm{H}_{26} \mathrm{Ge}+\mathrm{K}\right]^{+} 475.0878$; found 475.0879 .

The E configuration was assigned by analogy with E-8a. 
<smiles>OC/C(=C\c1ccccc1)c1ccccc1</smiles>

$13 a$
$\mathrm{C}_{27} \mathrm{H}_{24} \mathrm{GeO}$
$\mathrm{M}=437.12 \mathrm{~g} \cdot \mathrm{mol}^{-1}$

\begin{abstract}
(Z)-3-phenyl-2-(triphenylgermyl)prop-2-en-1-ol (13a): Prepared according to GP I using 3phenylprop-2-yn-1-ol 9a (33 mg, 0.25 mmol, 1.0 equiv) as substrate, $\mathrm{Et}_{2} \mathrm{Zn}$ (1.0 M in hexane, $0.75 \mathrm{~mL}, 0.75 \mathrm{mmol}, 3.0$ equiv), $\mathrm{Ph}_{3} \mathrm{GeH}$ (153 mg, $0.50 \mathrm{mmol}, 2.0$ equiv), AIBN (10 mg, 0.06 $\mathrm{mmol}, 25 \mathrm{~mol} \%)$ in heptane $(0.64 \mathrm{~mL})$ and a reaction time of $2 \mathrm{~h}$ at $100{ }^{\circ} \mathrm{C}$. Purification of the crude product $\left(Z / E>98: 2\right.$ ) by flash chromatography on silica gel (eluent toluene/ $E t_{2} \mathrm{O}=90: 10$ ) afforded analytically pure $13 \mathrm{a}(96 \mathrm{mg}, 87 \%, Z / E>98: 2)$ as a colorless liquid.
\end{abstract}

${ }^{1} \mathrm{H}$ NMR $\left(400 \mathrm{MHz}, \mathrm{CDCl}_{3}\right): \delta 7.74(\mathrm{~s}, 1 \mathrm{H}), 7.58-7.55(\mathrm{~m}, 6 \mathrm{H}), 7.40-7.31(\mathrm{~m}, 9 \mathrm{H}), 7.20-7.17$ (m, 2H), 7.00-6.95 (m, 1H), 6.93-6.89 (m, 2H), $4.39(\mathrm{~s}, 2 \mathrm{H}), 1.60$ (br s, 1H).

${ }^{13} \mathrm{C}\left\{{ }^{1} \mathrm{H}\right\}$ NMR $\left(100 \mathrm{MHz}, \mathrm{CDCl}_{3}\right): \delta 143.2,138.2,137.3,136.6,135.2,129.0,128.8,128.3$, $127.5,127.4,69.9$.

IR (neat): $v\left(\mathrm{~cm}^{-1}\right) 3302$ (br), 3022, 1485, 1430, 1088, 908, 732, 695.

HRMS (ESI): $m / z$ calculated for $\left[\mathrm{C}_{27} \mathrm{H}_{24} \mathrm{GeO}+\mathrm{K}\right]^{+} 477.0671$; found 477.0674 .

The Z configuration was established by analysis of NOESY correlations:

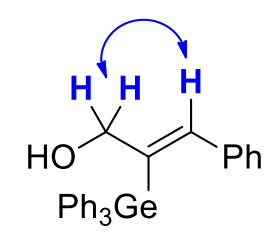

The chemical shift of the vinylic proton for the Z-isomer (7.74 ppm) is clearly distinct from the chemical shift of the vinylic proton for the E-isomer (6.98 ppm).<smiles>CC(O)C(=Cc1ccccc1)C(C)O</smiles>

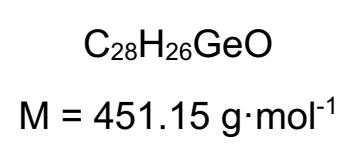

13b

(Z)-3-phenyl-2-(triphenylgermyl)prop-2-en-1-ol (13b): Prepared according to GP I using 4phenylbut-3-yn-2-ol $9 \mathrm{~b}$ (37 mg, $0.25 \mathrm{mmol}, 1.0$ equiv) as substrate, $\mathrm{Et}_{2} \mathrm{Zn}$ (1.0 M in hexane, $0.75 \mathrm{~mL}, 0.75 \mathrm{mmol}, 3.0$ equiv), $\mathrm{Ph}_{3} \mathrm{GeH}$ (153 mg, $0.50 \mathrm{mmol}, 2.0$ equiv), AlBN (10 mg, 0.06 $\mathrm{mmol}, 25 \mathrm{~mol} \%)$ in heptane $(0.64 \mathrm{~mL})$ and a reaction time of $2 \mathrm{~h}$ at $100{ }^{\circ} \mathrm{C}$. Purification of the crude product ( $Z / E>98: 2$ ) by flash chromatography on silica gel (eluent pentane/Et ${ }_{2} \mathrm{O} /$ toluene $=75: 15: 10)$ afforded analytically pure $13 \mathrm{~b}(94 \mathrm{mg}, 83 \%, Z / E>98: 2)$ as a colorless liquid. 
${ }^{1} \mathrm{H}$ NMR $\left(400 \mathrm{MHz}, \mathrm{CDCl}_{3}\right): \delta 7.84(\mathrm{~s}, 1 \mathrm{H}), 7.57-7.54(\mathrm{~m}, 6 \mathrm{H}), 7.36-7.30(\mathrm{~m}, 9 \mathrm{H}), 7.17-7.15$ (m, 2H), 6.94-6.90 (m, 1H), 6.87-6.83 (m, 2H), 4.60 (q, J = 6.4 Hz, 1H), 1.70 (br s, 1H), 1.36 (d, $J=6.4 \mathrm{~Hz}, 3 \mathrm{H})$.

${ }^{13} \mathrm{C}\left\{{ }^{1} \mathrm{H}\right\}$ NMR $\left(100 \mathrm{MHz}, \mathrm{CDCl}_{3}\right): \delta 143.2,140.6,137.3,137.1,135.3,128.9,128.85,128.2$, 127.4, 127.2, 72.4, 24.4 .

IR (neat): $v\left(\mathrm{~cm}^{-1}\right) 3322$ (br), 3023, 1485, 1430, 1088, 908, 732, 696.

HRMS (ESI): $\mathrm{m} / \mathrm{z}$ calculated for $\left[\mathrm{C}_{28} \mathrm{H}_{26} \mathrm{GeO}+\mathrm{K}\right]^{+} 491.0827$; found 491.0832 .

The Z configuration was assigned by analogy with Z-13a and by analysis of NOESY correlations:
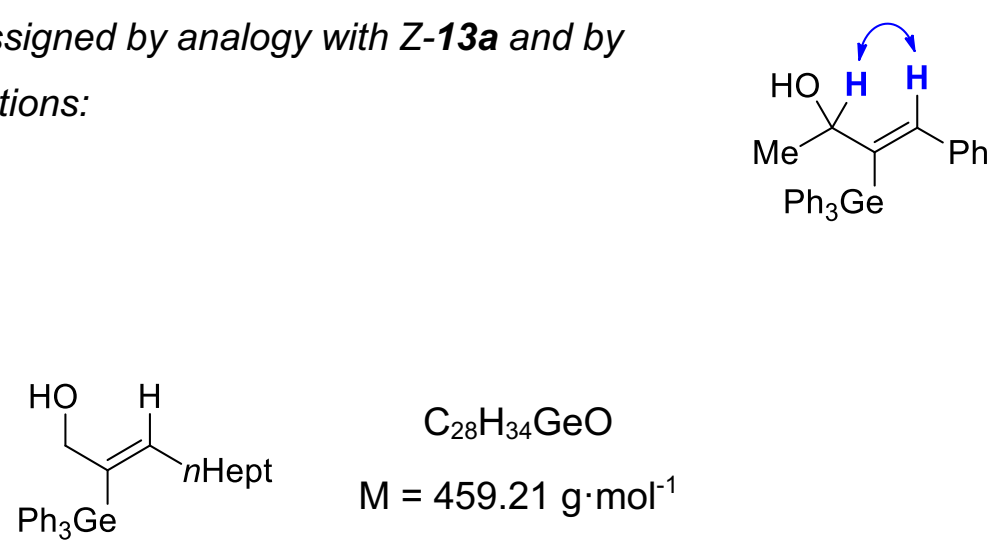

$13 c$

(Z)-2-(triphenylgermyl)dec-2-en-1-ol (13c): Prepared according to GP I using dec-2-yn-1-ol 9c (39 mg, $0.25 \mathrm{mmol}, 1.0$ equiv) as substrate, $\mathrm{Et}_{2} \mathrm{Zn}(1.0 \mathrm{M}$ in hexane, $0.75 \mathrm{~mL}, 0.75 \mathrm{mmol}$, 3.0 equiv), $\mathrm{Ph}_{3} \mathrm{GeH}$ (153 mg, $0.50 \mathrm{mmol}, 2.0$ equiv), AIBN (10 mg, $0.06 \mathrm{mmol}, 25 \mathrm{~mol} \%$ ) in heptane $(0.64 \mathrm{~mL})$ and a reaction time of $2 \mathrm{~h}$ at $100^{\circ} \mathrm{C}$. Purification of the crude product $((\beta / \alpha$ $=90(Z / E>98: 2): 10)$ by flash chromatography on silica gel (eluent pentane/toluene/ $\mathrm{Et}_{2} \mathrm{O}=$ 80:10:10) afforded analytically pure $13 \mathrm{c} \mathrm{(92} \mathrm{mg,} \mathrm{80 \% ,} \mathrm{Z/E} \mathrm{>} \mathrm{98:2)} \mathrm{as} \mathrm{a} \mathrm{colorless} \mathrm{liquid.}$

${ }^{1} \mathrm{H}$ NMR $\left(400 \mathrm{MHz}, \mathrm{CDCl}_{3}\right): \delta 7.63-7.61(\mathrm{~m}, 6 \mathrm{H}), 7.41-7.37(\mathrm{~m}, 9 \mathrm{H}), 6.55(\mathrm{t}, J=7.4 \mathrm{~Hz}, 1 \mathrm{H})$, $4.19(\mathrm{~s}, 2 \mathrm{H}), 1.94(\mathrm{q}, J=7.4 \mathrm{~Hz}, 2 \mathrm{H}), 1.34(\mathrm{br} \mathrm{s}, 1 \mathrm{H}), 1.26-0.93(\mathrm{~m}, 10 \mathrm{H}), 0.87(\mathrm{t}, J=7.2 \mathrm{~Hz}$, $3 \mathrm{H})$.

${ }^{13} \mathrm{C}\left\{{ }^{1} \mathrm{H}\right\}$ NMR $\left(100 \mathrm{MHz}, \mathrm{CDCl}_{3}\right): \delta 145.5,136.9,135.8,135.2,129.1,128.4,69.2,33.4,31.8$, 29.3, 29.2, 29.1, 22.7, 14.2 .

IR (neat): $v\left(\mathrm{~cm}^{-1}\right) 3328$ (br), 2923, 2853, 1623, 1430, 1088, 733, 698.

HRMS (ESI): $m / z$ calculated for $\left[\mathrm{C}_{28} \mathrm{H}_{34} \mathrm{GeO}+\mathrm{K}\right]^{+} 499.1453$; found 499.1454.

The $Z$ configuration was established by analysis of NOESY correlations:

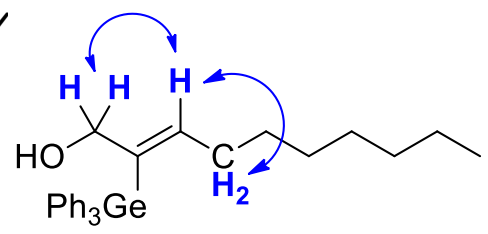




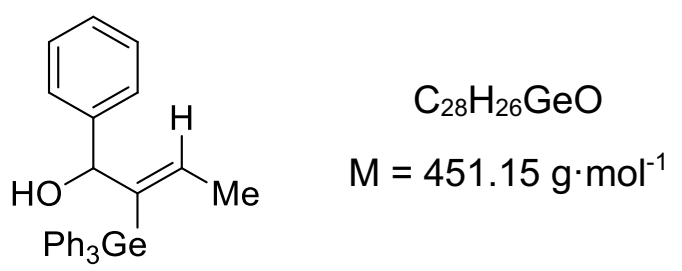

\section{$13 d$}

(Z)-1-phenyl-2-(triphenylgermyl)but-2-en-1-ol (13d): Prepared according to GP I using 1phenylbut-2-yn-1-ol 9d (37 mg, 0.25 mmol, 1.0 equiv) as substrate, $\mathrm{Et}_{2} Z \mathrm{n}$ (1.0 $\mathrm{M}$ in hexane, $0.75 \mathrm{~mL}, 0.75 \mathrm{mmol}, 3.0$ equiv), $\mathrm{Ph}_{3} \mathrm{GeH}$ (153 mg, $0.50 \mathrm{mmol}, 2.0$ equiv), AIBN (10 mg, 0.06 $\mathrm{mmol}, 25 \mathrm{~mol} \%)$ in heptane $(0.64 \mathrm{~mL})$ and a reaction time of $2 \mathrm{~h}$ at $100{ }^{\circ} \mathrm{C}$. Purification of the crude product $(Z / E>98: 2)$ by flash chromatography on silica gel (eluent pentane/Et ${ }_{2} \mathrm{O} /$ toluene $=75: 15: 10)$ afforded analytically pure $13 \mathrm{~d}(58 \mathrm{mg}, 51 \%$, Z/E > 98:2) as a white paste.

${ }^{1}$ H NMR (400 MHz, $\left.\mathrm{CDCl}_{3}\right): \delta$ 7.48-7.45 (m, 6H), 7.39-7.30 (m, 9H), 7.20-7.17 (m, 3H), 7.10$7.07(\mathrm{~m}, 2 \mathrm{H}), 6.66$ (qd, $J=6.9 \mathrm{~Hz}, J=1.2 \mathrm{~Hz}, 1 \mathrm{H}), 5.33$ (s, 1H), 1.87 (br s, 1H), 1.65 (dd, $J=$ $6.9 \mathrm{~Hz}, J=0.9 \mathrm{~Hz}, 3 \mathrm{H})$.

${ }^{13} \mathrm{C}\left\{{ }^{1} \mathrm{H}\right\}$ NMR $\left(100 \mathrm{MHz}, \mathrm{CDCl}_{3}\right): \delta 142.6,140.2,139.0,137.0,135.2,128.9,128.2,128.1$, 127.1, 126.9, 78.6, 19.5 .

IR (neat): $v\left(\mathrm{~cm}^{-1}\right) 3413$ (br), 3066, 3049, 1485, 1449, 1089, 734, 698.

HRMS (ESI): $m / z$ calculated for $\left[\mathrm{C}_{28} \mathrm{H}_{26} \mathrm{GeO}+\mathrm{Na}\right]^{+}$475.1088; found 475.1093.

The Z configuration was assigned by analogy with Z-13c.

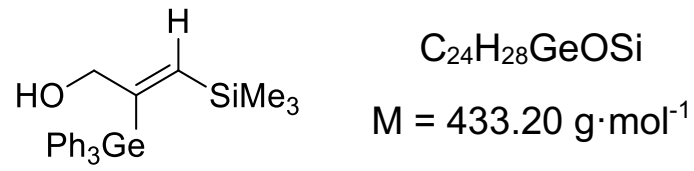

$13 e$

(Z)-3-(trimethylsilyl)-2-(triphenylgermyl)prop-2-en-1-ol (13e): Prepared according to GP I using 3-(trimethylsilyl)prop-2-yn-1-ol $9 e\left(32 \mathrm{mg}, 0.25 \mathrm{mmol}, 1.0\right.$ equiv) as substrate, $\mathrm{Et}_{2} \mathrm{Zn}$ (1.0 $\mathrm{M}$ in hexane, $0.75 \mathrm{~mL}, 0.75 \mathrm{mmol}, 3.0$ equiv), $\mathrm{Ph}_{3} \mathrm{GeH}$ (153 mg, $0.50 \mathrm{mmol}, 2.0$ equiv), AIBN $(10 \mathrm{mg}, 0.06 \mathrm{mmol}, 25 \mathrm{~mol} \%)$ in heptane $(0.64 \mathrm{~mL})$ and a reaction time of $2 \mathrm{~h}$ at $100{ }^{\circ} \mathrm{C}$. Purification of the crude product ( $Z / E>98: 2$ ) by flash chromatography on silica gel (eluent pentane/toluene/Et $\left.{ }_{2} \mathrm{O}=50: 45: 5\right)$ afforded analytically pure $13 \mathrm{e}(70 \mathrm{mg}, 65 \%, \mathrm{Z} / \mathrm{E}>98: 2)$ as a colorless liquid.

${ }^{1} \mathrm{H}$ NMR (400 MHz, $\left.\mathrm{CDCl}_{3}\right): \delta$ 7.62-7.59 (m, 6H), 7.43-7.36 (m, 9H), $6.93(\mathrm{~s}, 1 \mathrm{H}), 4.16(\mathrm{~s}, 2 \mathrm{H})$, 1.49 (br s, 1H), $-0.22(\mathrm{~s}, 9 \mathrm{H})$.

${ }^{13} \mathrm{C}\left\{{ }^{1} \mathrm{H}\right\}$ NMR $\left(100 \mathrm{MHz}, \mathrm{CDCl}_{3}\right): \delta 152.9,144.1,137.3,135.4,129.2,128.3,72.3,-0.2$. 
IR (neat): $v\left(\mathrm{~cm}^{-1}\right) 3325$ (br), 2997, 2952, 1430, 1247, 836, 732, 697.

HRMS (ESI): $m / z$ calculated for $\left[\mathrm{C}_{24} \mathrm{H}_{28} \mathrm{GeOSi}+\mathrm{Na}\right]^{+} 457.1013$; found 457.1019 .

The $Z$ configuration was confirmed by analysis of NOESY correlations:
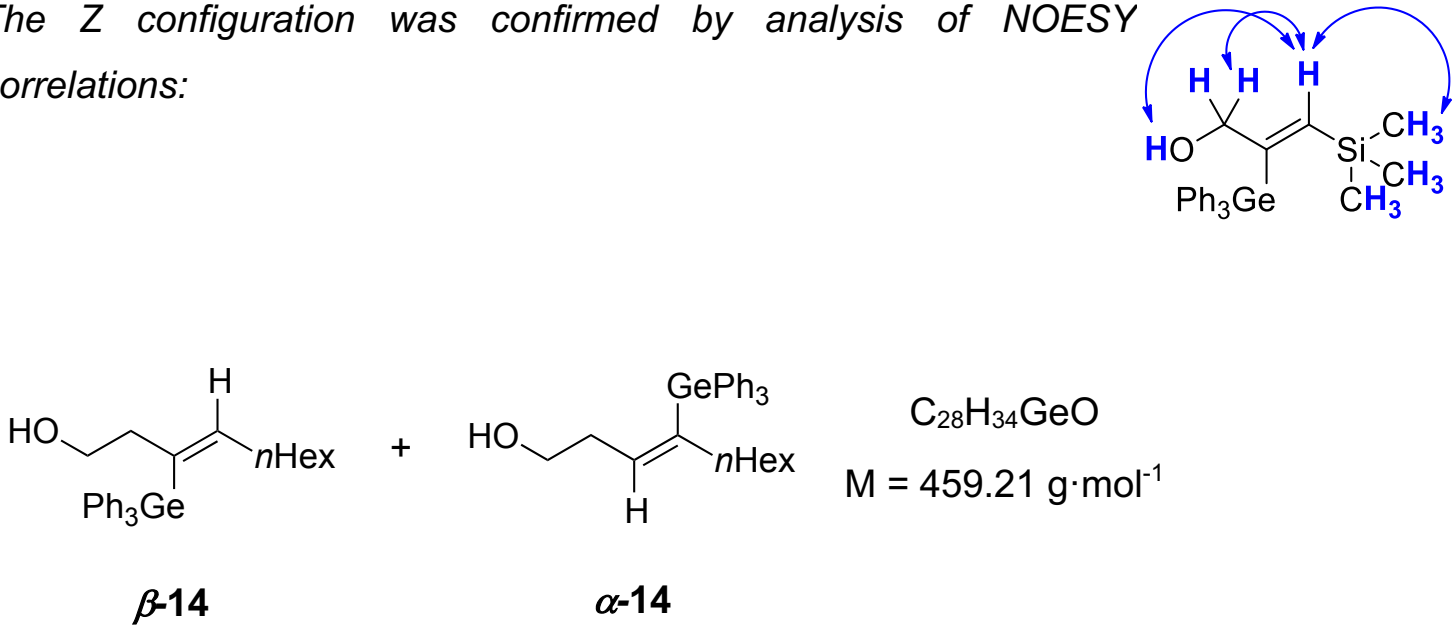

(Z)-3-(triphenylgermyl)dec-3-en-1-ol $(\beta$-14) and (Z)-4-(triphenylgermyl)dec-3-en-1-ol ( $\alpha$ 14): Prepared according to GP I using dec-3-yn-1-ol 10 (39 mg, $0.25 \mathrm{mmol}, 1.0$ equiv) as substrate, $\mathrm{Et}_{2} \mathrm{Zn}\left(1.0 \mathrm{M}\right.$ in hexane, $0.75 \mathrm{~mL}, 0.75 \mathrm{mmol}, 3.0$ equiv), $\mathrm{Ph}_{3} \mathrm{GeH}$ (153 mg, 0.50 $\mathrm{mmol}, 2.0$ equiv), AIBN (10 mg, $0.06 \mathrm{mmol}, 25 \mathrm{~mol} \%)$ in heptane $(0.64 \mathrm{~mL})$ and a reaction time of $2 \mathrm{~h}$ at $100{ }^{\circ} \mathrm{C}$. Purification of the crude product (mixture of regioisomers in ratio: $\beta-14 / \alpha-14$ $=57(Z / E=98: 2): 43(Z / E=98: 2)$ ) by flash chromatography on silica gel (eluent pentane/ $\mathrm{Et}_{2} \mathrm{O} /$ toluene $=75: 15: 10$ ) afforded an analytically pure fraction of the major regioisomer $\beta-14(20 \mathrm{mg}, 18 \%, Z / E>98: 2)$ and a second fraction containing a mixture of $\beta-14$ and $\alpha-14$ (45 mg, 39\%, $\beta-14 / \alpha-14=39(Z / E$ > 98:2):61(Z/E > 98:2)).

$\beta-14:$

Colorless liquid

${ }^{1} \mathrm{H}$ NMR $\left(400 \mathrm{MHz}, \mathrm{CDCl}_{3}\right): \delta 7.56-7.53(\mathrm{~m}, 6 \mathrm{H}), 7.39-7.35(\mathrm{~m}, 9 \mathrm{H}), 6.38(\mathrm{tt}, J=7.3 \mathrm{~Hz}, \mathrm{~J}=$ $1.2 \mathrm{~Hz}, 1 \mathrm{H}$ ), $3.40(\mathrm{t}, J=6.5 \mathrm{~Hz}, 2 \mathrm{H}), 2.44-2.40(\mathrm{~m}, 2 \mathrm{H}), 1.88(\mathrm{q}, J=7.3 \mathrm{~Hz}, 2 \mathrm{H}), 1.25$ (br s, $1 \mathrm{H}), 1.16-1.08(\mathrm{~m}, 4 \mathrm{H}), 1.01-0.87(\mathrm{~m}, 4 \mathrm{H}), 0.79(\mathrm{t}, J=7.3 \mathrm{~Hz}, 3 \mathrm{H})$.

${ }^{13} \mathrm{C}\left\{{ }^{1} \mathrm{H}\right\}$ NMR $\left(100 \mathrm{MHz}, \mathrm{CDCl}_{3}\right): \delta 147.2,137.2,135.2,132.0,129.0,128.4,62.0,41.8,34.0$, 31.7, 29.5, 29.0, 22.6, 14.1 .

IR (neat): $v\left(\mathrm{~cm}^{-1}\right) 3322$ (br), 2925, 2854, 1431, 1089, 1043, 735, 699.

HRMS (ESI): $m / z$ calculated for $\left[\mathrm{C}_{28} \mathrm{H}_{34} \mathrm{GeO}+\mathrm{Na}\right]^{+} 483.1714$; found 483.1713 .

The $Z$ configuration was assigned by analogy with Z-13c.

$\alpha-14$ :

${ }^{1} \mathrm{H}$ NMR $\left(400 \mathrm{MHz}, \mathrm{CDCl}_{3}\right): \delta$ 7.58-7.54 (m, 6H), 7.39-7.35 (m, 9H), $6.26(\mathrm{t}, J=7.3 \mathrm{~Hz}, 1 \mathrm{H})$, $3.38(\mathrm{t}, J=6.8 \mathrm{~Hz}, 2 \mathrm{H}), 2.18-2.09(\mathrm{~m}, 4 \mathrm{H}), 1.25-0.95(\mathrm{~m}, 9 \mathrm{H}), 0.80(\mathrm{t}, J=7.3 \mathrm{~Hz}, 3 \mathrm{H})$. 
${ }^{13} \mathrm{C}\left\{{ }^{1} \mathrm{H}\right\}$ NMR $\left(100 \mathrm{MHz}, \mathrm{CDCl}_{3}\right): \delta 140.0,138.5,137.5,135.2,129.0,128.3,62.5,39.2,37.0$, $31.5,30.5,28.9,22.6,14.2$.<smiles>C=CCC(=C(c1ccccc1)c1ccccc1)c1ccccc1</smiles>

15
$\mathrm{C}_{35} \mathrm{H}_{30} \mathrm{Ge}$
$M=523.25 \mathrm{~g} \cdot \mathrm{mol}^{-1}$

(Z)-(1,2-diphenylpenta-1,4-dien-1-yl)triphenylgermanetriphenylgermane (15): Prepared according to GP II using diphenylacetylene $1 \mathrm{a}(45 \mathrm{mg}, 0.25 \mathrm{mmol}, 1.0$ equiv) as substrate, $\mathrm{Et}_{2} \mathrm{Zn}\left(1.0 \mathrm{M}\right.$ in hexane, $0.75 \mathrm{~mL}, 0.75 \mathrm{mmol}, 3.0$ equiv), $\mathrm{Ph}_{3} \mathrm{GeH}$ (153 mg, $0.50 \mathrm{mmol}, 2.0$ equiv), AIBN (10 mg, $0.06 \mathrm{mmol}, 25 \mathrm{~mol} \%)$ in dry THF $(0.64 \mathrm{~mL})$ and a reaction time of $1 \mathrm{~h}$ at $80{ }^{\circ} \mathrm{C}$ for the germylzincation step and $\mathrm{CuCN} \cdot 2 \mathrm{LiCl}(1.0 \mathrm{M}$ in THF, $0.75 \mathrm{~mL}, 0.75 \mathrm{mmol}, 3.0$ equiv) and allyl bromide (212 mg, $1.75 \mathrm{mmol}, 7.0$ equiv) for the electrophilic substitution step. Purification of the crude product by flash chromatography on silica gel (eluent pentane/toluene $=92: 8)$ afforded analytically pure 15 (76 mg, 58\%, Z/E > 98:2) as a white solid; $\mathrm{mp} 89-92{ }^{\circ} \mathrm{C}$.

${ }^{1} \mathrm{H}$ NMR $\left(400 \mathrm{MHz}, \mathrm{CDCl}_{3}\right):$ ס 7.04-6.99 (m, 9H), 6.97-6.91 (m, 8H), 6.89-6.86 (m, 1H), 6.85$6.80(\mathrm{~m}, 4 \mathrm{H}), 6.65-6.54(\mathrm{~m}, 3 \mathrm{H}), 5.45$ (ddt, $J=17.0 \mathrm{~Hz}, J=10.1 \mathrm{~Hz}, J=6.8 \mathrm{~Hz}, 1 \mathrm{H}$ ), 4.68 (ddt, $J=10.1 \mathrm{~Hz}, J=2.1 \mathrm{~Hz}, J=1.1 \mathrm{~Hz}, 1 \mathrm{H}), 4.61\left(\mathrm{dq}_{\mathrm{app}}, J=17.0 \mathrm{~Hz}, J=1.6 \mathrm{~Hz}, 1 \mathrm{H}\right), 2.94(\mathrm{dt}, J=$ $6.8 \mathrm{~Hz}, J=1.4 \mathrm{~Hz}, 2 \mathrm{H})$.

${ }^{13} \mathrm{C}\left\{{ }^{1} \mathrm{H}\right\}$ NMR $\left(100 \mathrm{MHz}, \mathrm{CDCl}_{3}\right): \delta 153.0,143.1,141.8,138.4,137.8,135.9,135.3,129.1$, $128.8,128.2,127.9,127.7,127.4,126.9,125.6,116.0,42.3$.

IR (neat): $v\left(\mathrm{~cm}^{-1}\right)$ 3138, 3050, 1596, 1485, 1430, 909, 731, 695.

HRMS (APCI): $m / z$ calculated for $\left[\mathrm{C}_{35} \mathrm{H}_{30} \mathrm{Ge}+\mathrm{H}\right]^{+} 525.1632$; found 525.1633.

The structure of 15 was determined by X-ray crystallographic analysis (CCDC 2077611).

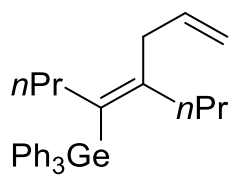

16
$\mathrm{C}_{29} \mathrm{H}_{34} \mathrm{Ge}$
$\mathrm{M}=$
$455.22 \mathrm{~g} \cdot \mathrm{mol}^{-1}$

(E)-triphenyl(5-propylocta-4,7-dien-4-yl)germane (16): Prepared according to GP II using 4-octyne $4 a$ (28 mg, $0.25 \mathrm{mmol}, 1.0$ equiv) as substrate, $\mathrm{Et}_{2} \mathrm{Zn}(1.0 \mathrm{M}$ in hexane, $0.75 \mathrm{~mL}, 0.75$ mmol, 3.0 equiv), $\mathrm{Ph}_{3} \mathrm{GeH}$ (153 mg, $0.50 \mathrm{mmol}, 2.0$ equiv), AIBN (41 mg, $0.25 \mathrm{mmol}, 100$ $\mathrm{mol} \%)$ in dry THF $(0.64 \mathrm{~mL})$ and microwave-heating at $80^{\circ} \mathrm{C}(50 \mathrm{~W})$ for $1 \mathrm{~h}$ (normal absorption level) for the germylzincation step and $\mathrm{CuCN} \cdot 2 \mathrm{LiCl}(1.0 \mathrm{M}$ in THF, $0.75 \mathrm{~mL}, 0.75 \mathrm{mmol}, 3.0$ equiv) and allyl bromide (212 mg, $1.75 \mathrm{mmol}, 7.0$ equiv) for the electrophilic substitution step. 
Purification of the crude product $(E / Z=89: 11)$ by flash chromatography on silica gel (eluent pentane/toluene $=92: 8)$ afforded $16(65 \mathrm{mg}, 58 \%, E / Z>98: 2) ; \mathrm{mp} \mathrm{71-74}{ }^{\circ} \mathrm{C}$.

${ }^{1} \mathrm{H}$ NMR (400 MHz, CDCl 3 ): $\delta$ 7.59-7.54 (m, 6H), 7.39-7.34 (m, 9H), 5.89 (ddt, $J=17.1 \mathrm{~Hz}, J$ $=10.0 \mathrm{~Hz}, J=6.5 \mathrm{~Hz}, 1 \mathrm{H}), 5.15\left(\mathrm{dq}_{\mathrm{app}}, J=17.1 \mathrm{~Hz}, J=1.7 \mathrm{~Hz}, 1 \mathrm{H}\right), 5.09(\mathrm{dq} \mathrm{app}, J=10.0 \mathrm{~Hz}$, $J=1.7 \mathrm{~Hz}, 1 \mathrm{H}), 3.04(\mathrm{dt}, J=6.5 \mathrm{~Hz}, J=1.7 \mathrm{~Hz}, 2 \mathrm{H}), 2.15-2.11(\mathrm{~m}, 2 \mathrm{H}), 1.95-1.91(\mathrm{~m}, 2 \mathrm{H})$, 1.24-1.14 (m, 2H), 1.05-0.96 (m, 2H), $0.57(\mathrm{t}, J=7.3 \mathrm{~Hz}, 3 \mathrm{H}), 0.23(\mathrm{t}, J=7.3 \mathrm{~Hz}, 3 \mathrm{H})$.

${ }^{13} \mathrm{C}\left\{{ }^{1} \mathrm{H}\right\}$ NMR $\left(100 \mathrm{MHz}, \mathrm{CDCl}_{3}\right): \delta 149.4,138.6,137.1,135.4,131.9,128.7,128.1,115.5$, 40.8, 35.8, 34.8, 23.7, 21.6, 14.0, 13.9.

IR (neat): $v\left(\mathrm{~cm}^{-1}\right) 2957,2929,2869,1430,1089,911,734,699$.

HRMS (APCI): $m / z$ calculated for $\left[\mathrm{C}_{29} \mathrm{H}_{34} \mathrm{Ge}-\mathrm{H}\right]^{+} 455.1789$; found 455.1790 .

The E configuration was determined by analysis of NOESY correlations:
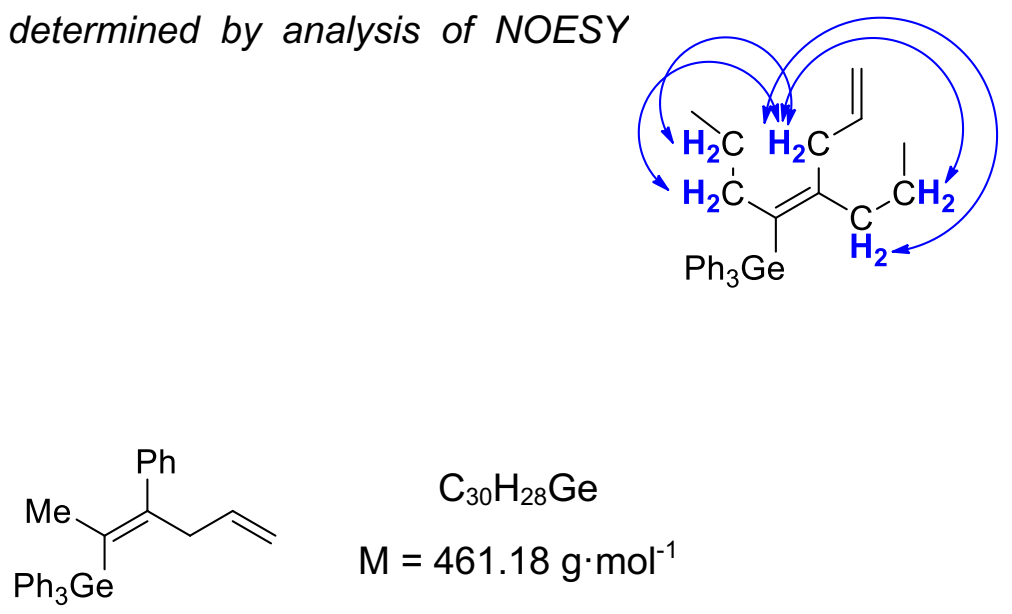

17

(E)-triphenyl(1-phenylprop-1-en-2-yl)germane (17): Prepared according to GP II using 1phenyl-1-propyne 7a (29 mg, $0.25 \mathrm{mmol}, 1.0$ equiv) as substrate, $\mathrm{Et}_{2} \mathrm{Zn}$ (1.0 $\mathrm{M}$ in hexane, 0.75 $\mathrm{mL}, 0.75 \mathrm{mmol}, 3.0$ equiv), $\mathrm{Ph}_{3} \mathrm{GeH}$ (153 mg, $0.50 \mathrm{mmol}, 2.0$ equiv), AIBN (4 mg, $0.03 \mathrm{mmol}$, $10 \mathrm{~mol} \%)$ in heptane $(0.64 \mathrm{~mL})$ and a reaction time of $2 \mathrm{~h}$ at $100{ }^{\circ} \mathrm{C}$ for the germylzincation step and CuCN.2LiCl (1.0 M in THF, $0.75 \mathrm{~mL}, 0.75 \mathrm{mmol}, 3.0$ equiv) and allyl bromide (212 $\mathrm{mg}, 1.75 \mathrm{mmol}, 7.0$ equiv) for the electrophilic substitution step. Purification of the crude product by flash chromatography on silica gel (eluent pentane/toluene $=92: 8$ ) afforded analytically pure 17 (107 mg, 93\%, E/Z > 98:2) as a white solid; mp $116-119^{\circ} \mathrm{C}$.

${ }^{1} \mathrm{H}$ NMR $\left(400 \mathrm{MHz}, \mathrm{CDCl}_{3}\right): \delta 7.74-7.70(\mathrm{~m}, 6 \mathrm{H}), 7.49-7.42(\mathrm{~m}, 11 \mathrm{H}), 7.34-7.30(\mathrm{~m}, 1 \mathrm{H}), 7.28-$ $7.25(\mathrm{~m}, 2 \mathrm{H}), 5.29$ (ddt, $J=17.0 \mathrm{~Hz}, J=10.1 \mathrm{~Hz}, J=6.8 \mathrm{~Hz}, 1 \mathrm{H}$ ), 4.65 (ddt, $J=10.1 \mathrm{~Hz}, J=$ $2.2 \mathrm{~Hz}, J=1.2 \mathrm{~Hz}, 1 \mathrm{H}), 4.38\left(\mathrm{dq}_{\mathrm{app}}, J=17.0 \mathrm{~Hz}, J=1.6 \mathrm{~Hz}, 1 \mathrm{H}\right), 3.17\left(\mathrm{dq}_{\mathrm{app}}, J=6.8 \mathrm{~Hz}, J=\right.$ $1.2 \mathrm{~Hz}, 2 \mathrm{H}), 1.73(\mathrm{t}, J=1.2 \mathrm{~Hz}, 3 \mathrm{H})$.

${ }^{13} \mathrm{C}\left\{{ }^{1} \mathrm{H}\right\}$ NMR $\left(100 \mathrm{MHz}, \mathrm{CDCl}_{3}\right): \delta 151.3,142.6,137.8,135.4,135.0,129.0,128.8,128.76$, $128.4,128.2,126.5,116.1,44.7,22.5$. 
IR (neat): $v\left(\mathrm{~cm}^{-1}\right)$ 3068, 3018, 1484, 1430, 1069, 908, 731, 697.

HRMS (ESI): $m / z$ calculated for $\left[\mathrm{C}_{30} \mathrm{H}_{28} \mathrm{Ge}+\mathrm{K}\right]^{+} 501.1034$; found 501.1038.

The structure of 17 was determined by X-ray crystallographic analysis (CCDC 2077612).

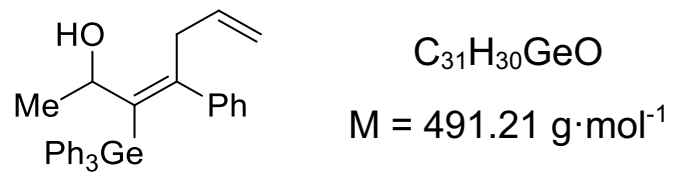

18

(Z)-4-phenyl-3-(triphenylgermyl)hepta-3,6-dien-2-ol (18): Prepared according to GP II using 4-phenylbut-3-yn-2-ol 9b (37 mg, $0.25 \mathrm{mmol}, 1.0$ equiv) as substrate, $\mathrm{Et}_{2} \mathrm{Zn}$ (1.0 $\mathrm{M}$ in hexane, $0.75 \mathrm{~mL}, 0.75 \mathrm{mmol}, 3.0$ equiv), $\mathrm{Ph}_{3} \mathrm{GeH}$ (153 mg, $0.50 \mathrm{mmol}, 2.0$ equiv), AIBN (10 $\mathrm{mg}, 0.06 \mathrm{mmol}, 25 \mathrm{~mol} \%)$ in heptane $(0.64 \mathrm{~mL})$ and a reaction time of $2 \mathrm{~h}$ at $100{ }^{\circ} \mathrm{C}$ for the germylzincation step and $\mathrm{CuCN} \cdot 2 \mathrm{LiCl}(1.0 \mathrm{M}$ in THF, $0.75 \mathrm{~mL}, 0.75 \mathrm{mmol}, 3.0$ equiv) and allyl bromide (212 mg, $1.75 \mathrm{mmol}, 7.0$ equiv) for the electrophilic substitution step. Purification of the crude product by flash chromatography on silica gel (eluent pentane/ $\mathrm{Et}_{2} \mathrm{O} /$ toluene $=$ 80:10:10) afforded analytically pure 18 (91 mg, 74\%, Z/E > 98:2) as a white solid; mp 109-112 ${ }^{\circ} \mathrm{C}$.

${ }^{1} \mathrm{H}$ NMR $\left(400 \mathrm{MHz}, \mathrm{CDCl}_{3}\right): \delta$ 7.55-7.52 (m, 6H), 7.33-7.27 (m, 9H), $6.84(\mathrm{~d}, J=7.6 \mathrm{~Hz}, 2 \mathrm{H})$, 6.79-6.75 (m, 1H), $6.67(\mathrm{t}, J=7.6 \mathrm{~Hz}, 2 \mathrm{H}), 5.80$ (ddt, $J=16.6 \mathrm{~Hz}, J=10.6 \mathrm{~Hz}, J=6.4 \mathrm{~Hz}$, $1 \mathrm{H}), 5.15-5.05(\mathrm{~m}, 3 \mathrm{H}), 3.41-3.38(\mathrm{~m}, 2 \mathrm{H}), 1.65(\mathrm{br} \mathrm{s}, 1 \mathrm{H}), 1.40(\mathrm{~d}, J=6.4 \mathrm{~Hz}, 3 \mathrm{H})$.

${ }^{13} \mathrm{C}\left\{{ }^{1} \mathrm{H}\right\}$ NMR $\left(100 \mathrm{MHz}, \mathrm{CDCl}_{3}\right): \delta 150.9,142.6,141.5,139.3,135.4,135.1,128.8,128.3$, $127.9,127.2,126.7,116.1,70.6,41.1,24.6$.

IR (neat): $v\left(\mathrm{~cm}^{-1}\right) 3427$ (br), 3068, 3049, 1485, 1430, 909, 732, 697.

HRMS (APCI): $m / z$ calculated for $\left[\mathrm{C}_{31} \mathrm{H}_{30} \mathrm{GeO}+\mathrm{H}\right]^{+} 493.1581$; found 493.1583 .

The $Z$ configuration was determined by analysis of NOESY correlations:

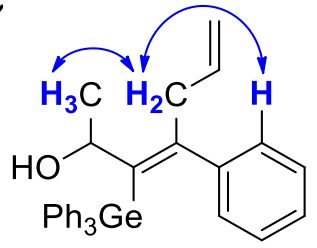


<smiles>C/C(=C(/C(=O)c1ccccc1)c1ccccc1)[PH](=O)c1ccccc1</smiles>

19

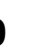

$$
\begin{gathered}
\mathrm{C}_{34} \mathrm{H}_{28} \mathrm{GeO} \\
M=525.23 \mathrm{~g} \cdot \mathrm{mol}^{-1}
\end{gathered}
$$

(Z)-1,2-diphenyl-3-(triphenylgermyl)but-2-en-1-one (19): Prepared according to GP II using 1-phenyl-1-propyne $7 \mathrm{a}$ (29 mg, $0.25 \mathrm{mmol}, 1.0$ equiv) as substrate, $\mathrm{Et}_{2} \mathrm{Zn}$ (1.0 M in hexane, $0.75 \mathrm{~mL}, 0.75 \mathrm{mmol}, 3.0$ equiv), $\mathrm{Ph}_{3} \mathrm{GeH}$ (153 mg, $0.50 \mathrm{mmol}, 2.0$ equiv), AlBN (4 mg, 0.03 $\mathrm{mmol}, 10 \mathrm{~mol} \%)$ in heptane $(0.64 \mathrm{~mL})$ and a reaction time of $2 \mathrm{~h}$ at $100{ }^{\circ} \mathrm{C}$ for the germylzincation step and $\mathrm{CuCN} \cdot 2 \mathrm{LiCl}(1.0 \mathrm{M}$ in THF, $0.75 \mathrm{~mL}, 0.75 \mathrm{mmol}, 3.0$ equiv) and benzoyl chloride (246 mg, $1.75 \mathrm{mmol}, 7.0$ equiv) for the electrophilic substitution step. Purification of the crude product by flash chromatography on silica gel (eluent pentane/toluene/ $\left.\mathrm{Et}_{2} \mathrm{O}=80: 18: 2\right)$ afforded analytically pure $19(83 \mathrm{mg}, 63 \%, \mathrm{Z} / \mathrm{E}>98: 2)$ as a white solid; mp $144-146^{\circ} \mathrm{C}$.

${ }^{1} \mathrm{H}$ NMR $\left(400 \mathrm{MHz}, \mathrm{CDCl}_{3}\right): \delta$ 7.59-7.56 (m, 6H), 7.50-7.48 (m, 2H), 7.41-7.35 (m, 4H), 7.32$7.25(\mathrm{~m}, 11 \mathrm{H}), 7.13-7.09(\mathrm{~m}, 2 \mathrm{H}), 2.11(\mathrm{~s}, 3 \mathrm{H})$.

${ }^{13} \mathrm{C}\left\{{ }^{1} \mathrm{H}\right\}$ NMR $\left(100 \mathrm{MHz}, \mathrm{CDCl}_{3}\right): \delta 196.0,153.1,140.5,137.6,136.7,136.2,135.6,132.2$, $129.9,129.4,128.8,128.6,128.0,127.7,22.1$. One $C$ is not observed.

IR (neat): $v\left(\mathrm{~cm}^{-1}\right) 3051,1659,1431,1260,1089,908,768,697$.

HRMS (APCl): $\mathrm{m} / \mathrm{z}$ calculated for $\left[\mathrm{C}_{34} \mathrm{H}_{28} \mathrm{GeO}+\mathrm{H}^{+}\right]^{+} 527.1425$; found 527.1415 .

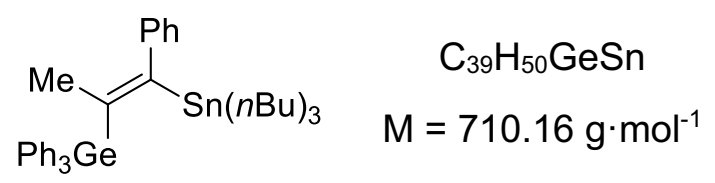

(Z)-triphenyl(1-phenyl-1-(tributylstannyl)prop-1-en-2-yl)germane $\quad$ (20): $\quad$ Prepared according to GP II using 1-phenyl-1-propyne $7 \mathrm{a}(29 \mathrm{mg}, 0.25 \mathrm{mmol}, 1.0$ equiv) as substrate, $\mathrm{Et}_{2} \mathrm{Zn}\left(1.0 \mathrm{M}\right.$ in hexane, $0.75 \mathrm{~mL}, 0.75 \mathrm{mmol}, 3.0$ equiv), $\mathrm{Ph}_{3} \mathrm{GeH}(153 \mathrm{mg}, 0.50 \mathrm{mmol}, 2.0$ equiv), AIBN ( $4 \mathrm{mg}, 0.03 \mathrm{mmol}, 10 \mathrm{~mol} \%)$ in heptane $(0.64 \mathrm{~mL})$ and a reaction time of $2 \mathrm{~h}$ at $100^{\circ} \mathrm{C}$ for the germylzincation step and CuCN.2LiCl $(1.0 \mathrm{M}$ in THF, $0.75 \mathrm{~mL}, 0.75 \mathrm{mmol}, 3.0$ equiv) and tributyltin chloride (570 mg, $1.75 \mathrm{mmol}, 7.0$ equiv) for the electrophilic substitution step. Purification of the crude product by flash chromatography on silica gel (eluent pentane/toluene $=95: 5)$ afforded a white solid containing 20 (105 mg, 59\%, Z/E > 98:2) and unidentified tributylstannyl-containing residues $(10 \mathrm{mg}) ; \mathrm{mp} 84-86{ }^{\circ} \mathrm{C}$. 
${ }^{1} \mathrm{H}$ NMR $\left(400 \mathrm{MHz}, \mathrm{CDCl}_{3}\right):$ $\mathbf{7} 7.68-7.66(\mathrm{~m}, 6 \mathrm{H}), 7.43-7.34(\mathrm{~m}, 11 \mathrm{H}), 7.19-7.15(\mathrm{~m}, 1 \mathrm{H}), 7.07-$ $7.04(\mathrm{~m}, 2 \mathrm{H}), 1.70-1.68(\mathrm{~m}, 3 \mathrm{H}), 1.05-1.00(\mathrm{~m}, 12 \mathrm{H}), 0.75-0.72(\mathrm{~m}, 9 \mathrm{H}), 0.39-0.22(\mathrm{~m}, 6 \mathrm{H})$. ${ }^{13} \mathrm{C}\left\{{ }^{1} \mathrm{H}\right\}$ NMR $\left(100 \mathrm{MHz}, \mathrm{CDCl}_{3}\right): \delta 163.7,148.1,144.4,138.2,135.5,129.0,128.3,128.27$, 126.7, 124.9, 29.1, 27.4, 24.8, 13.6, 11.9.

IR (neat): $v\left(\mathrm{~cm}^{-1}\right)$ 2954, 2920, 1953, 1882, 1431, 1088, 734, 699.

HRMS (APCI): $m / z$ calculated for $\left[\mathrm{C}_{39} \mathrm{H}_{50} \mathrm{Ge}+\mathrm{H}\right]^{+}-\mathrm{C}_{6} \mathrm{H}_{6}=\left[\mathrm{C}_{33} \mathrm{H}_{45} \mathrm{GeSn}\right]^{+}$633.1763; found 633.1756.

The $Z$ configuration was confirmed by analysis of NOESY correlations:

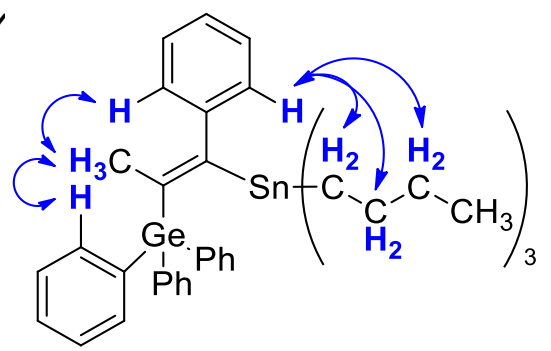


II.3 NMR Spectra for New Compounds 


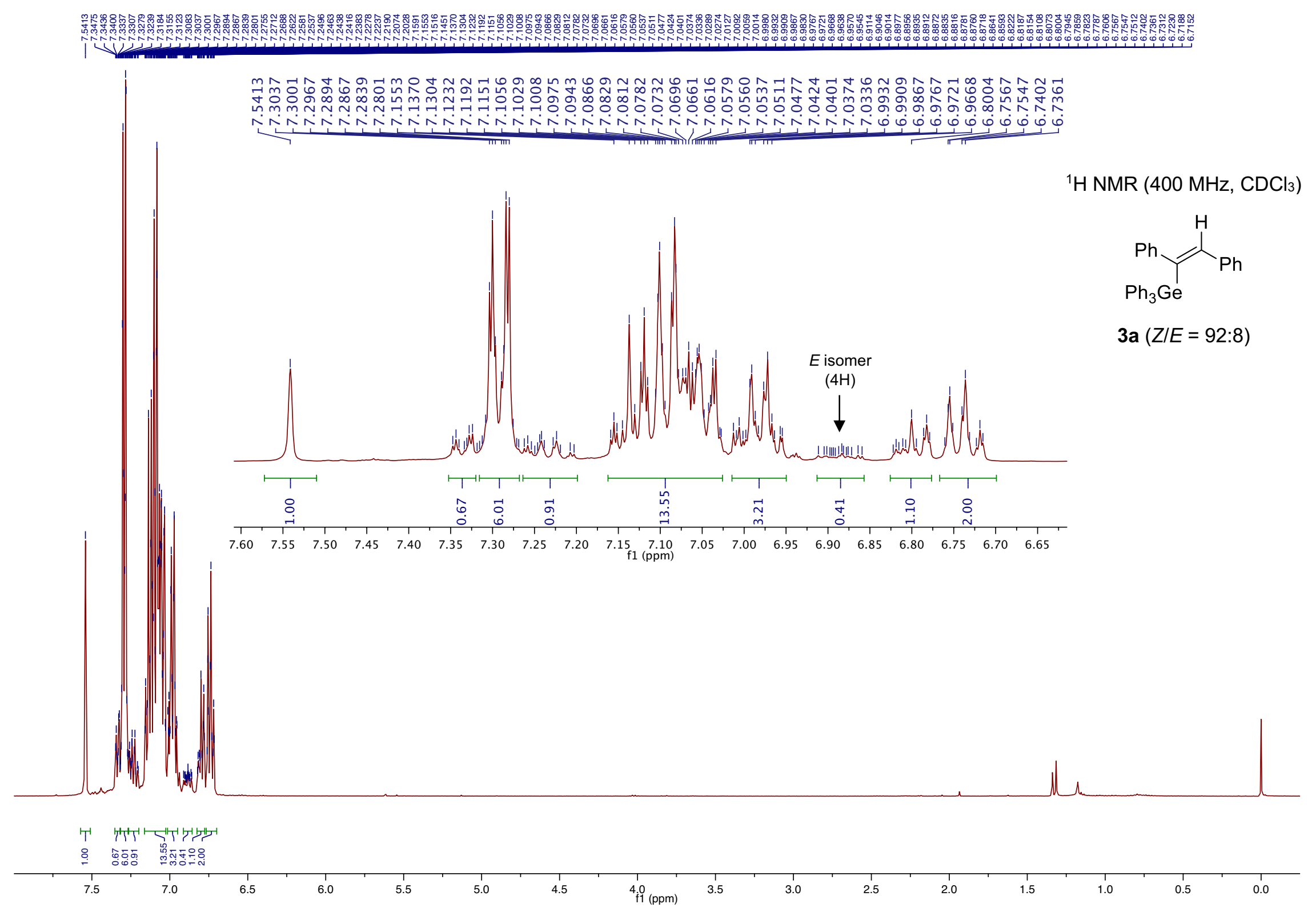



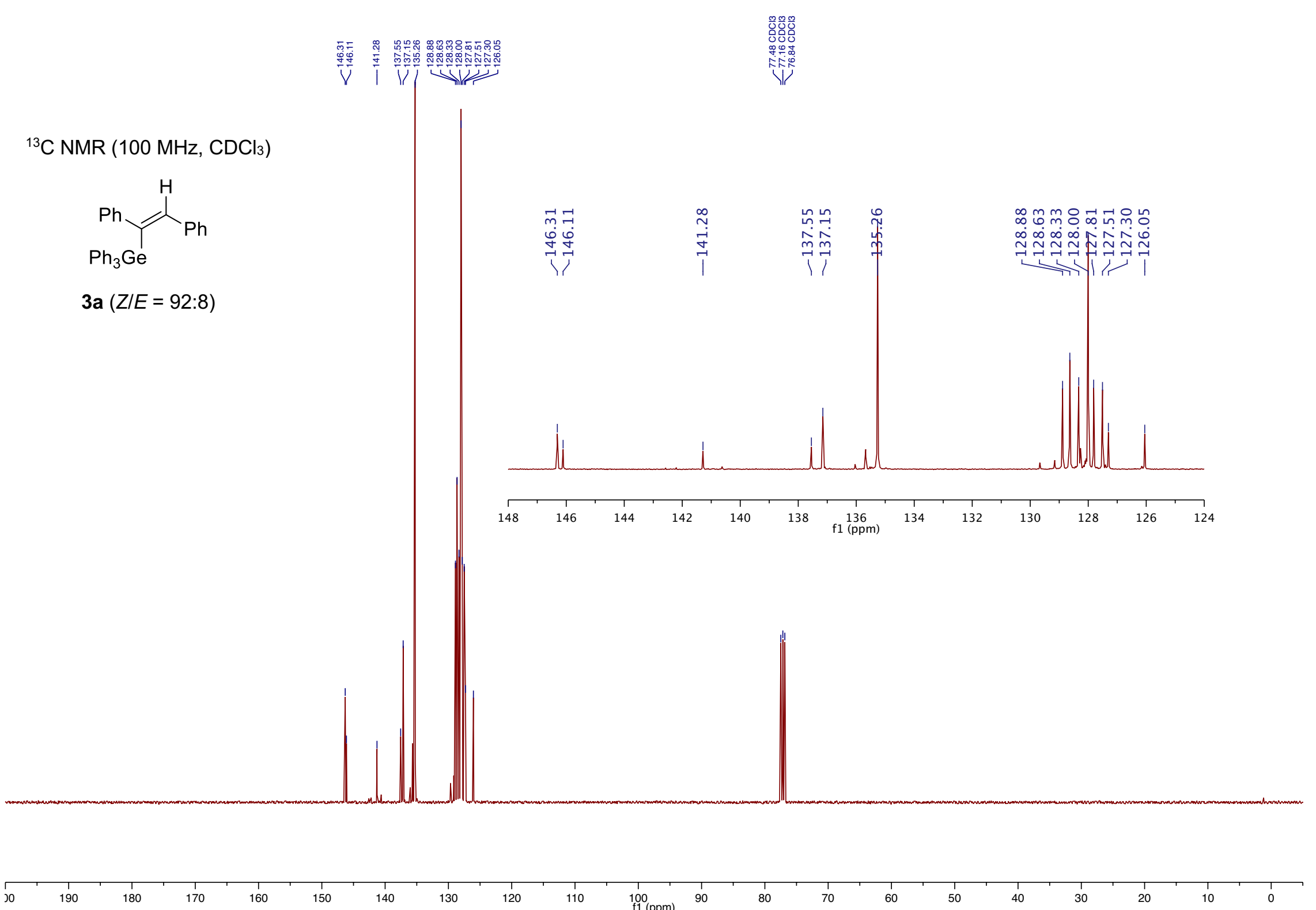

160

150

140

130

${ }_{\mathrm{f1}}^{100}(\mathrm{ppm})$

80

70

60

50 


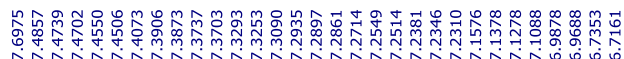
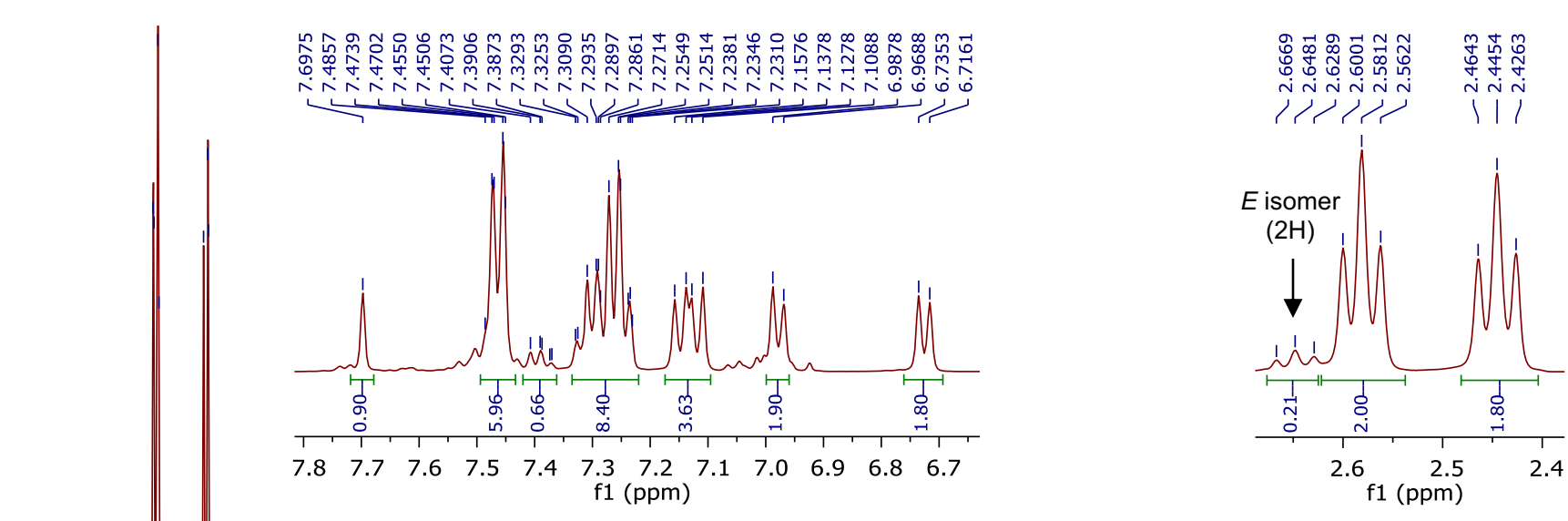

$\mathrm{Bu}$

3b $(Z / E=90: 10)$

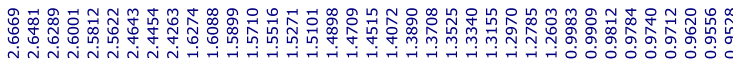

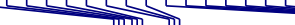

${ }^{1} \mathrm{H}$ NMR (400 MHz, $\left.\mathrm{CDCl}_{3}\right)$
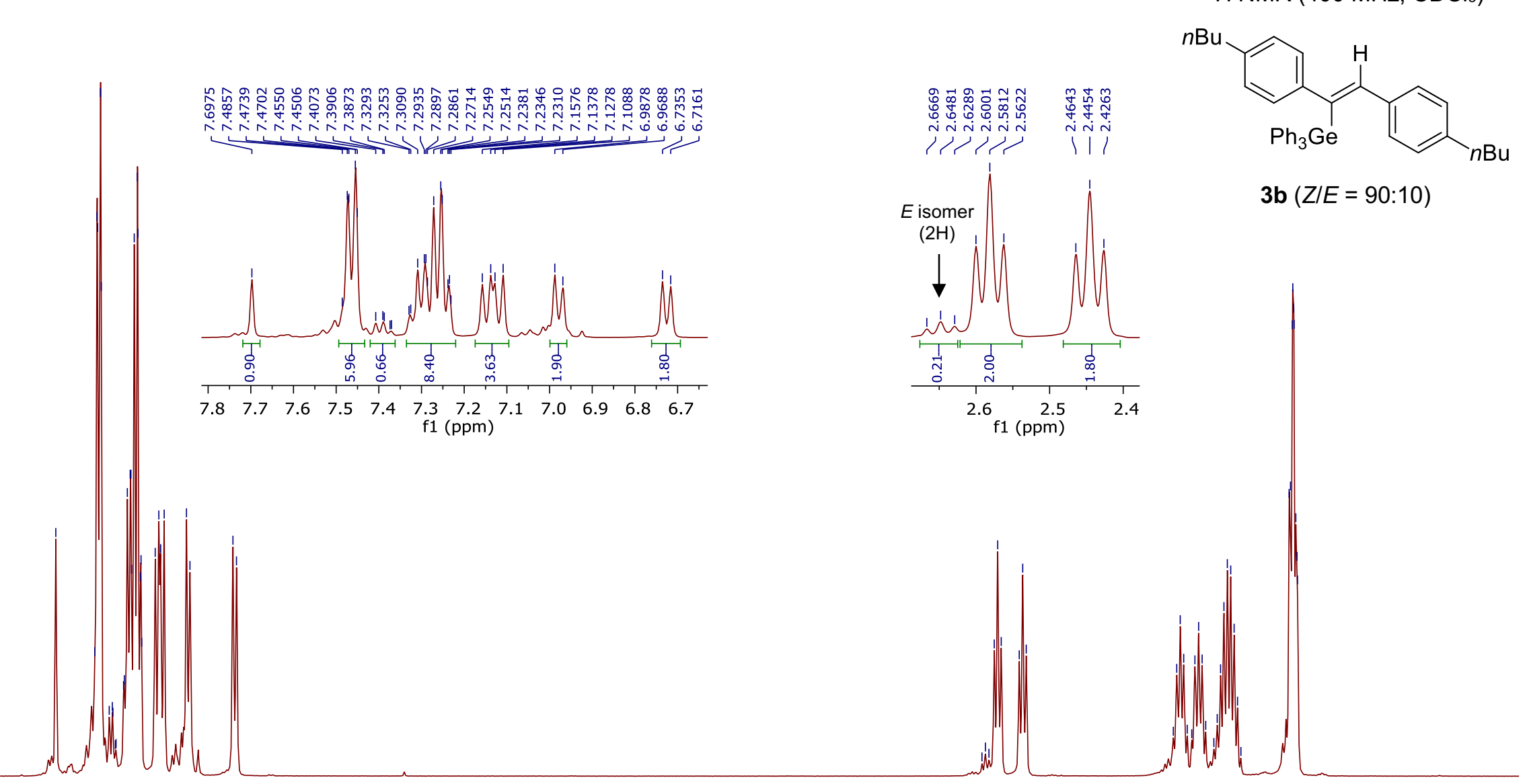

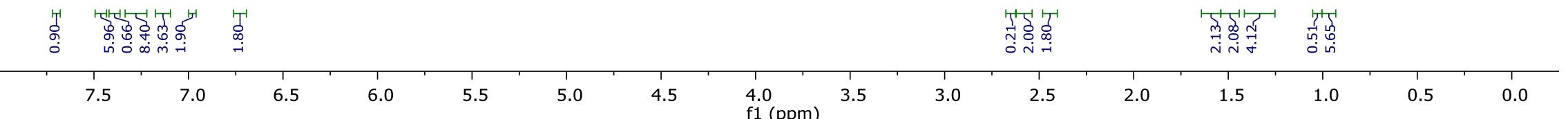



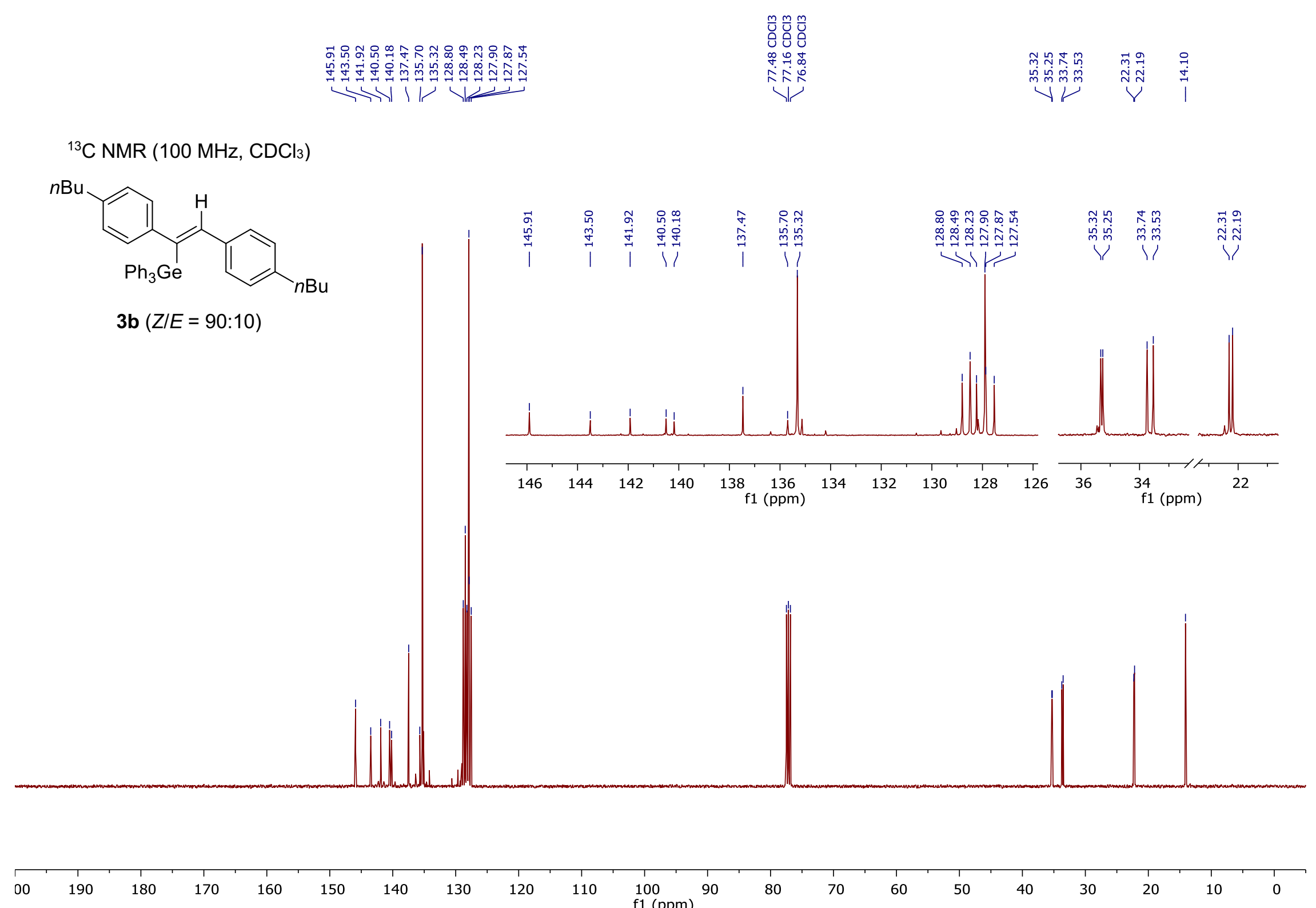


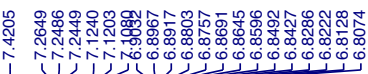

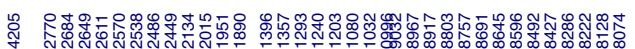

sim
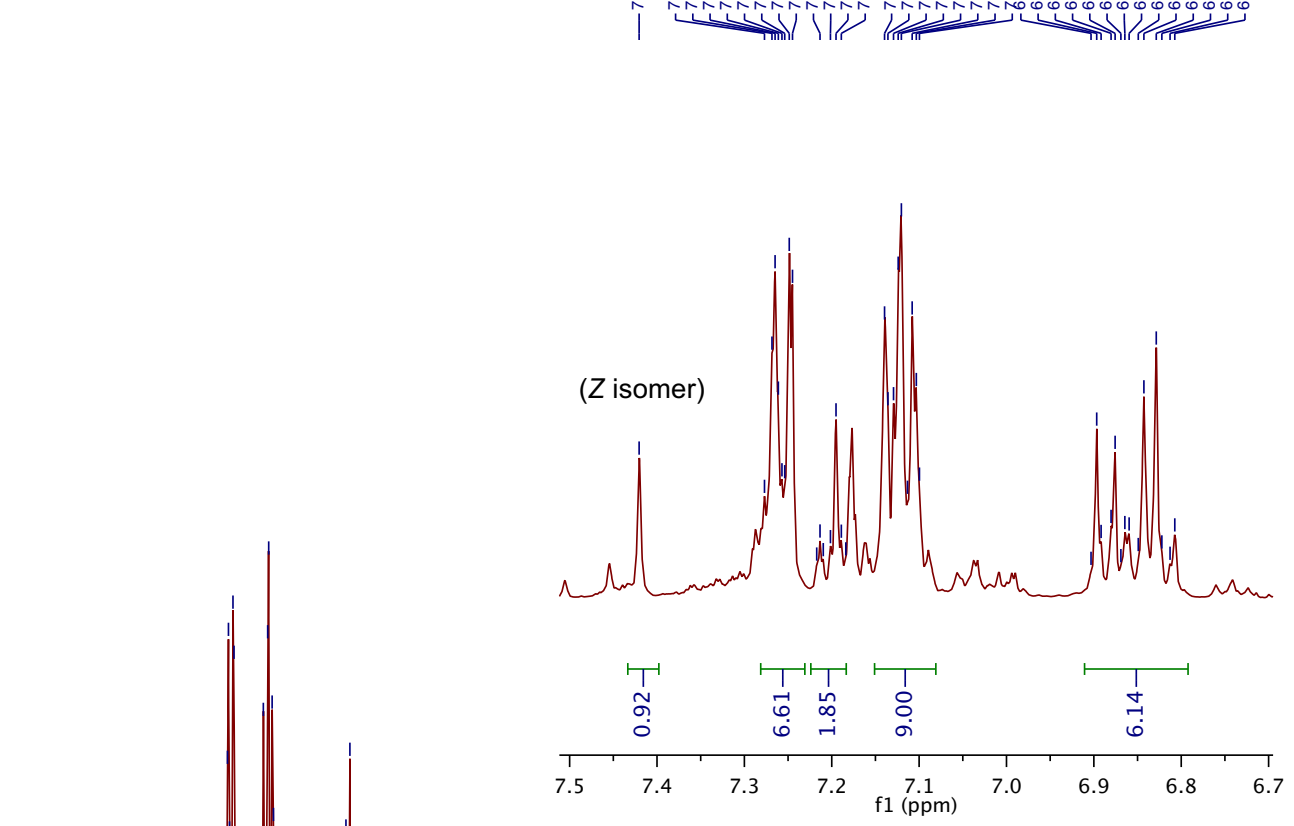

${ }^{1} \mathrm{H}$ NMR $\left(400 \mathrm{MHz}, \mathrm{CDCl}_{3}\right)$

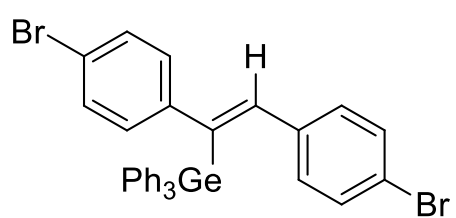

$3 c(Z / E=87: 13)$
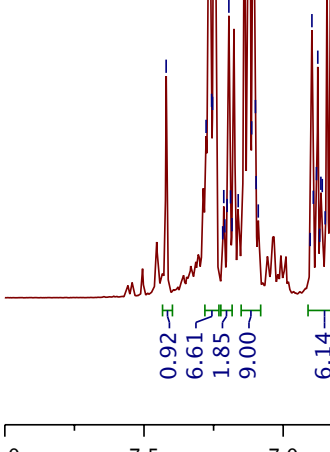

6.5

6.0

5.5

5.0

4.5

4.0
$\mathrm{f} 1(\mathrm{ppm})$

3.5

3.0

2.5

2.0 

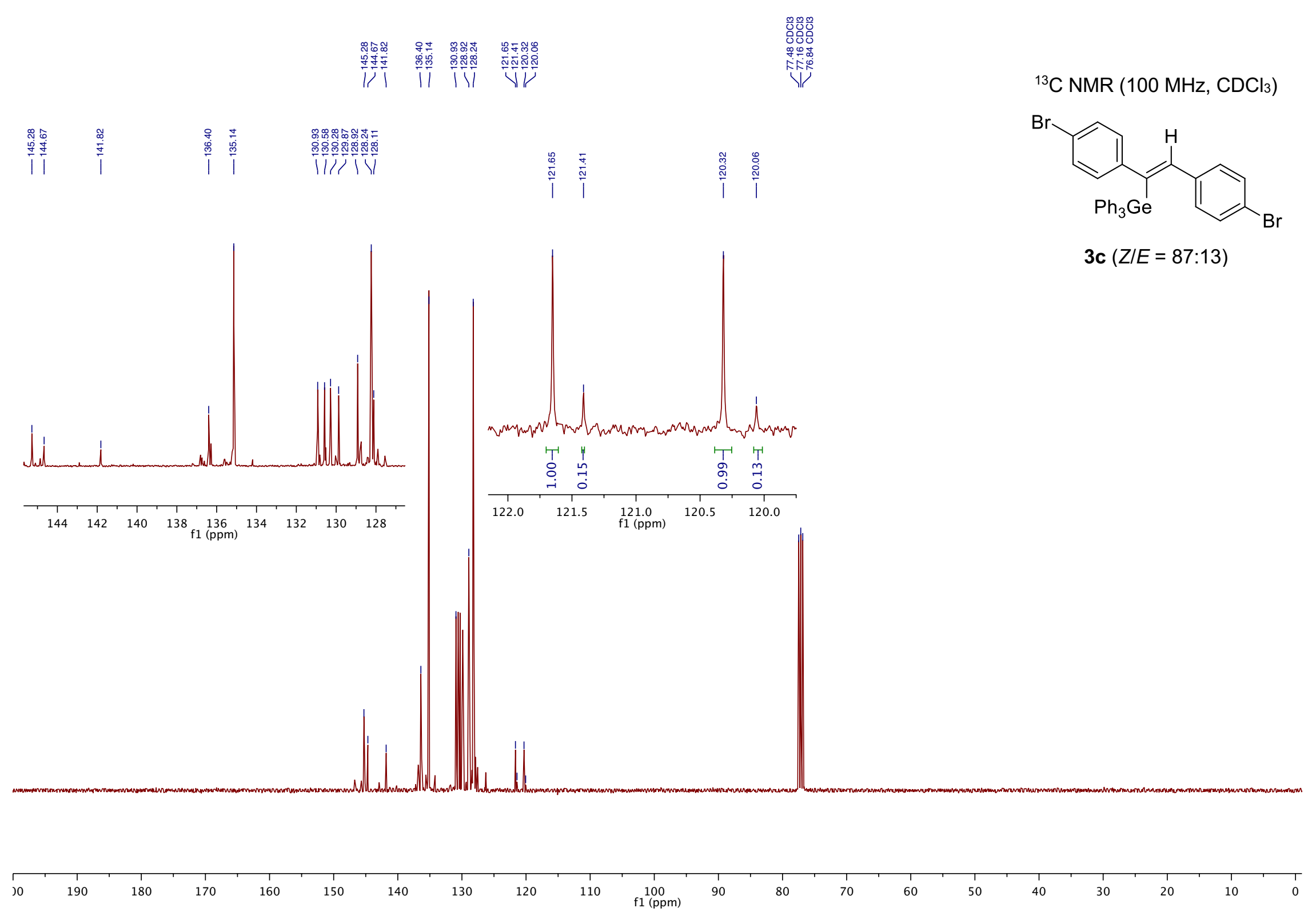


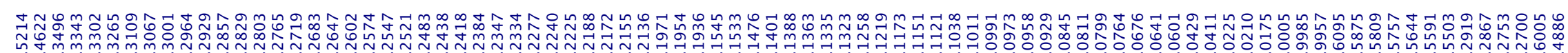

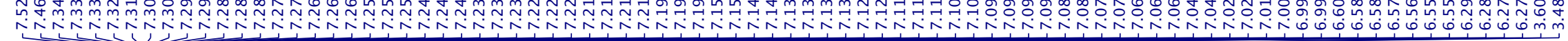

${ }^{1} \mathrm{H}$ NMR $\left(400 \mathrm{MHz}, \mathrm{CDCl}_{3}\right)$
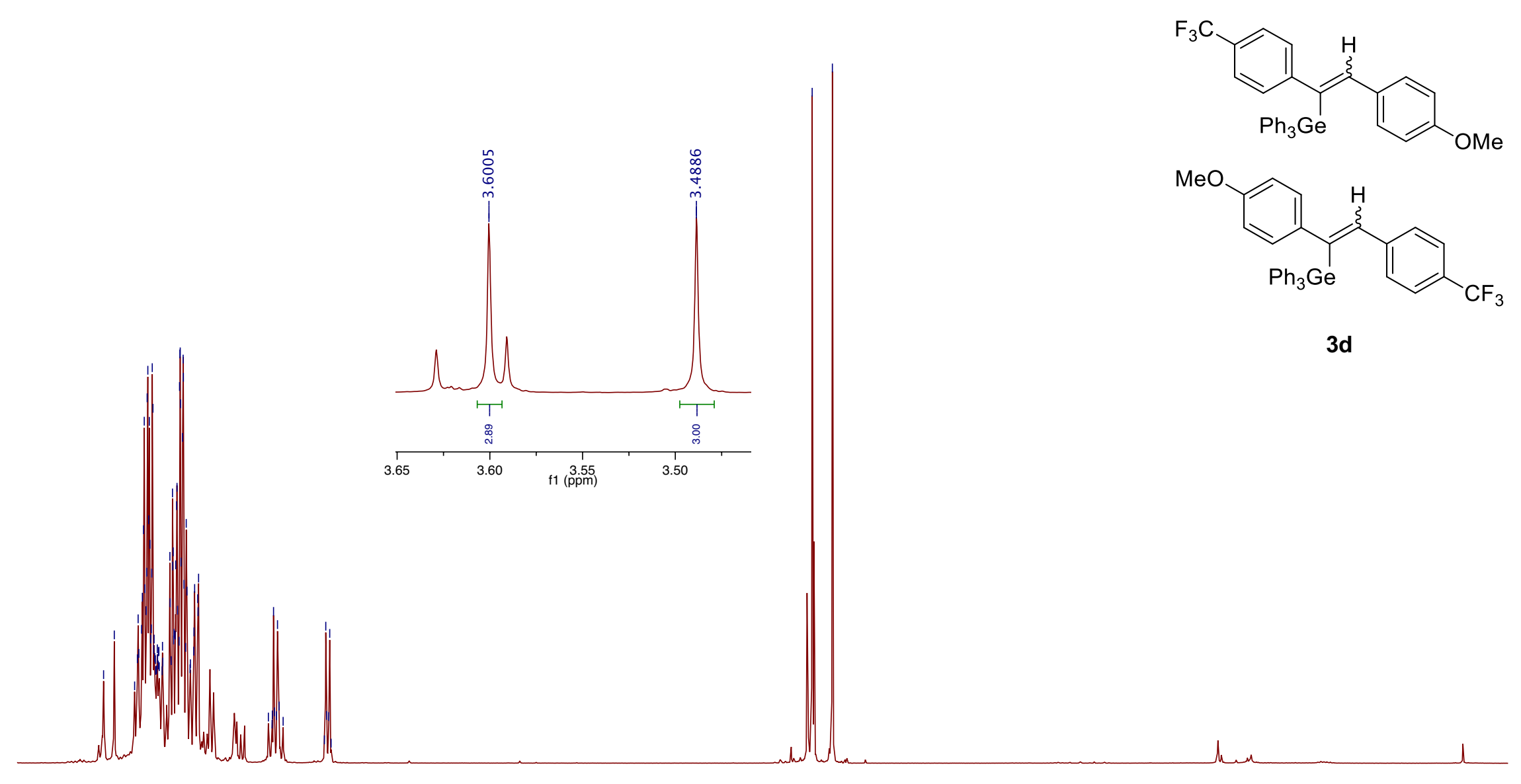

$3 d$

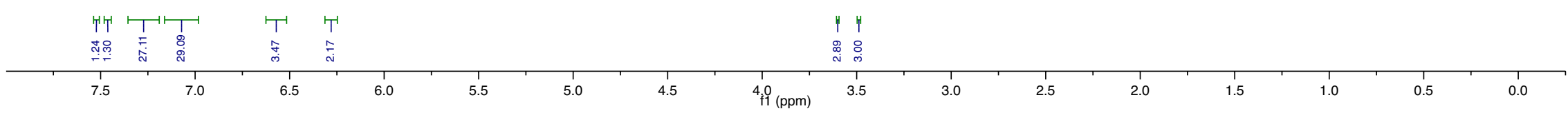



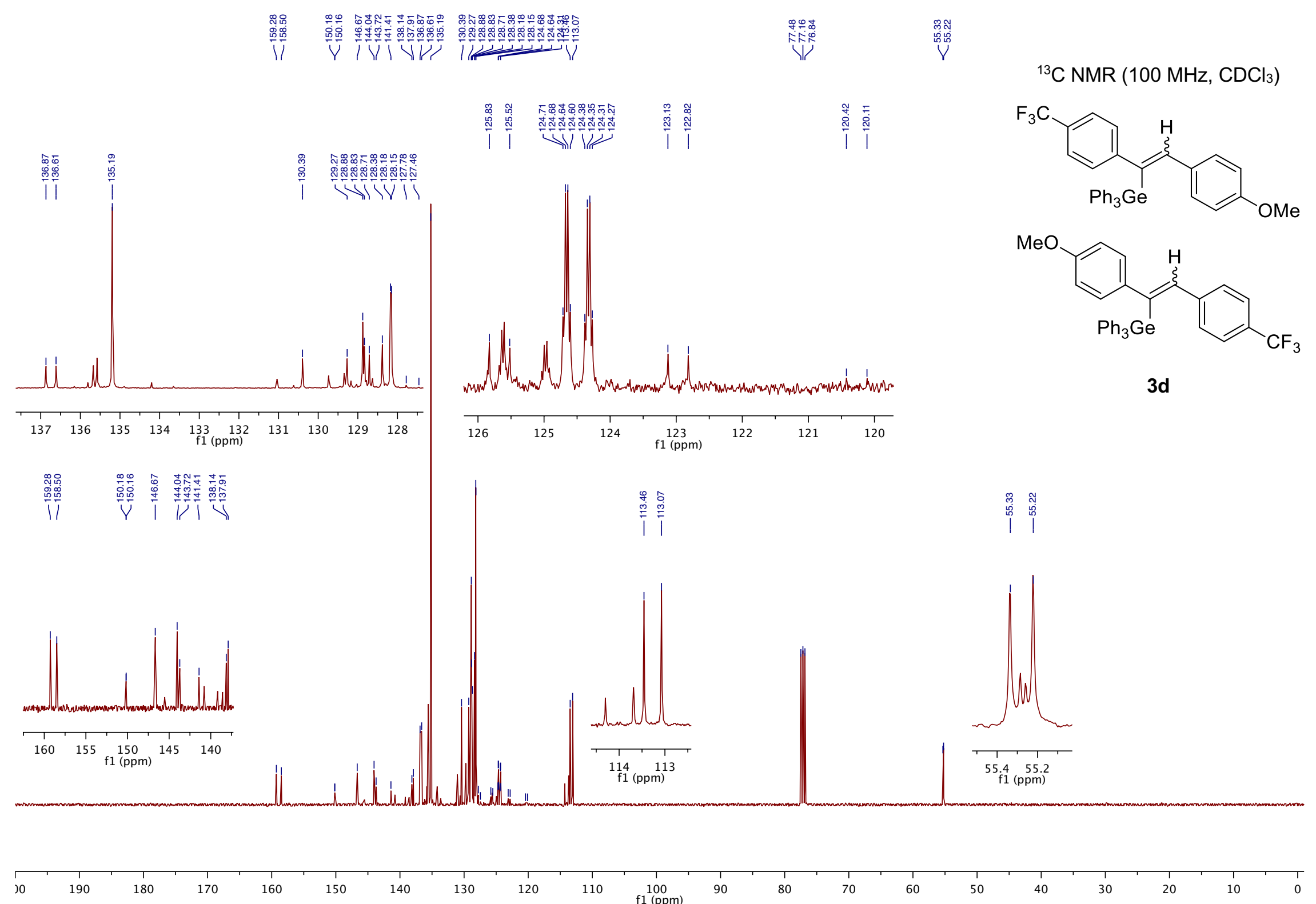


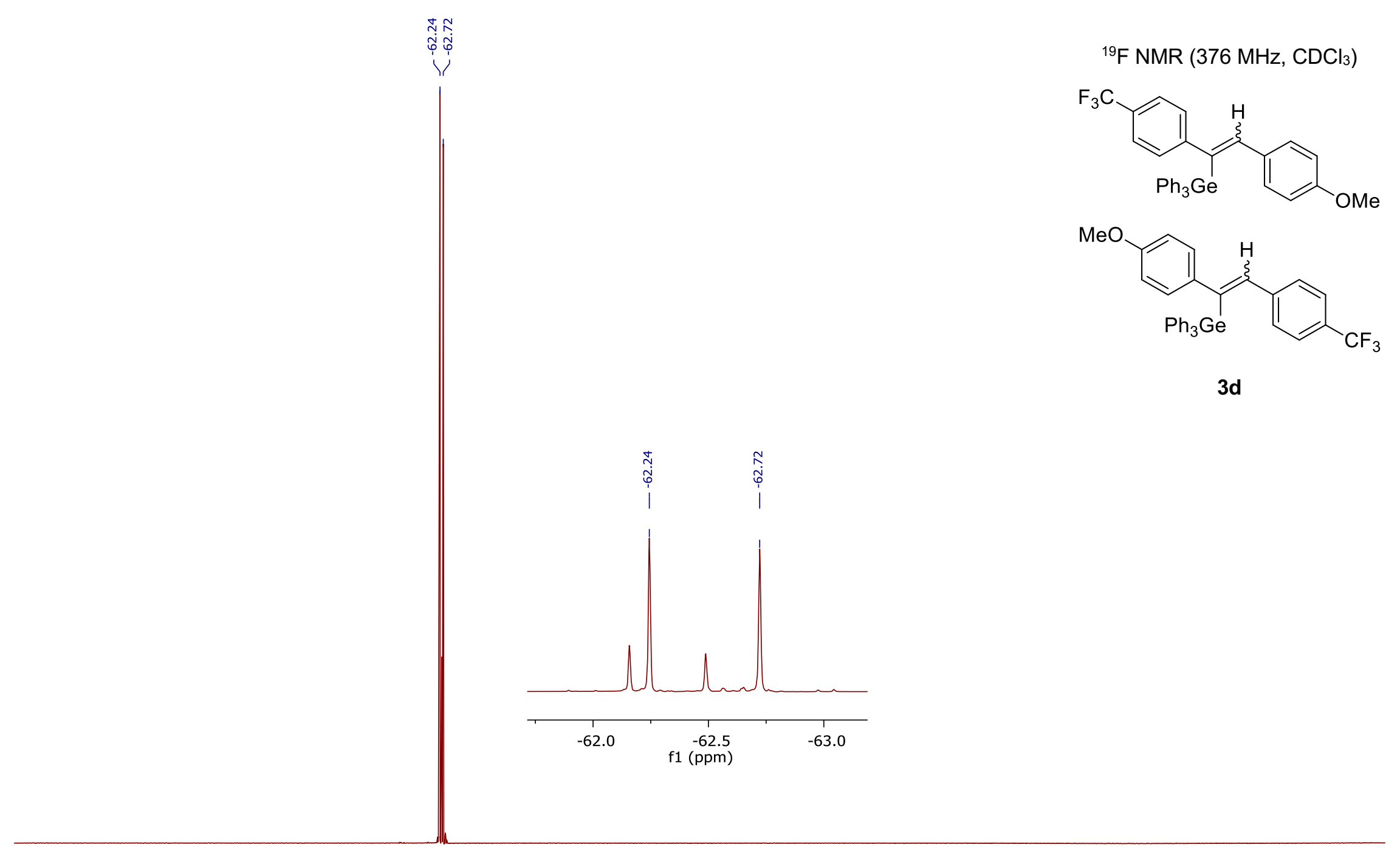

\begin{tabular}{|c|c|c|c|c|c|c|c|c|c|c|c|c|c|c|c|c|c|c|}
\hline-10 & -20 & -30 & -40 & -50 & -60 & -70 & -80 & -90 & $f 1 \stackrel{-100}{(\mathrm{ppm})}$ & -110 & -120 & -130 & -140 & -150 & -160 & -170 & -180 & -190 \\
\hline
\end{tabular}




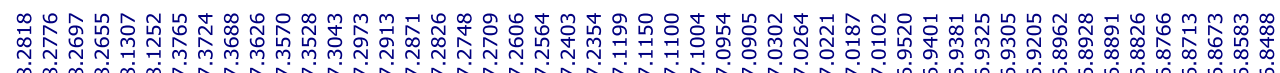

$\underbrace{-1}$

${ }^{1} \mathrm{H}$ NMR $\left(400 \mathrm{MHz}, \mathrm{CDCl}_{3}\right)$

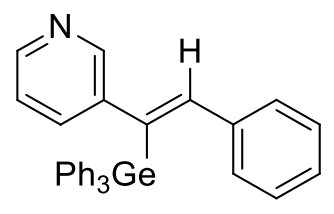

$\beta-3 e(Z / E>98: 2)$

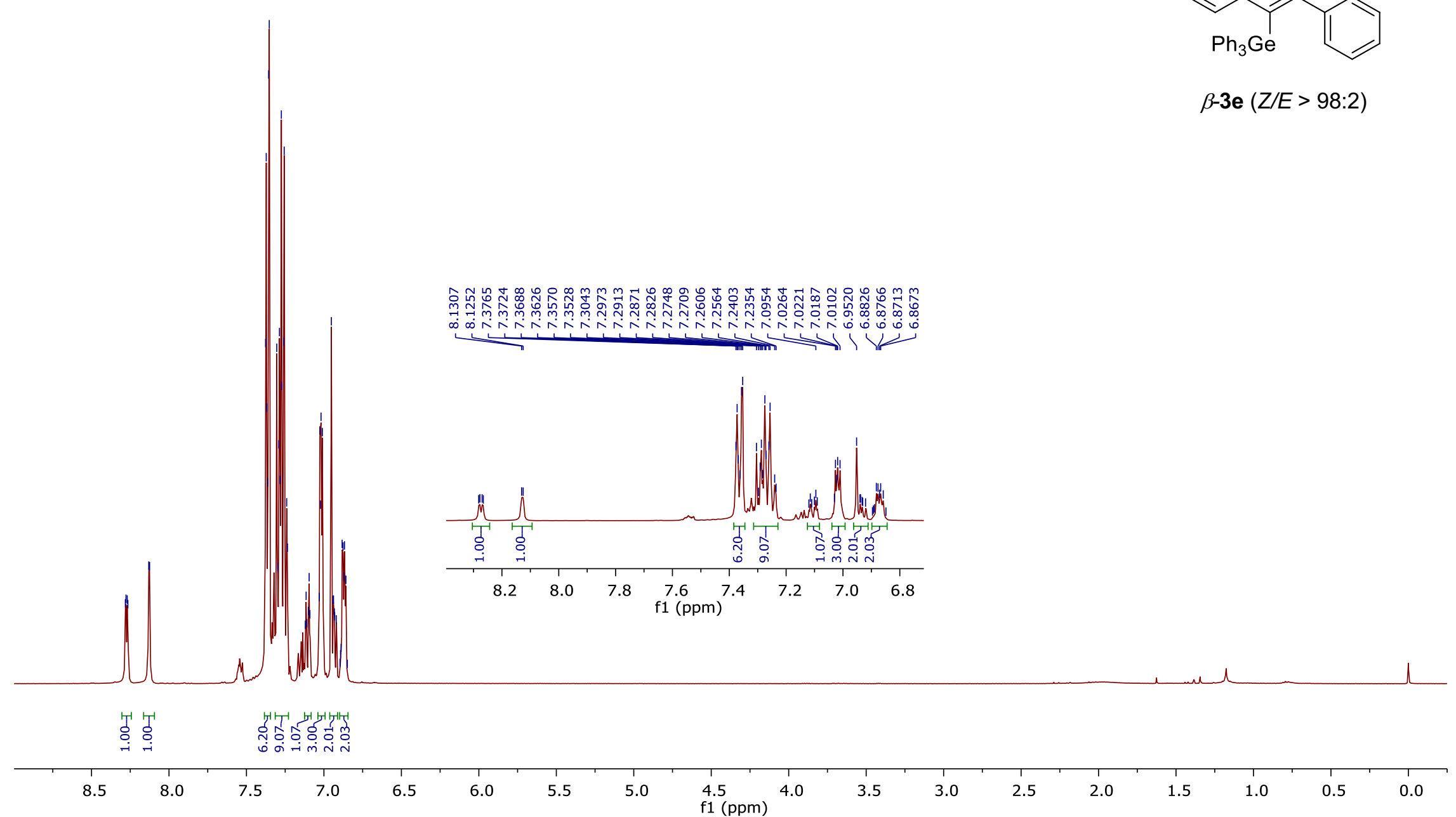




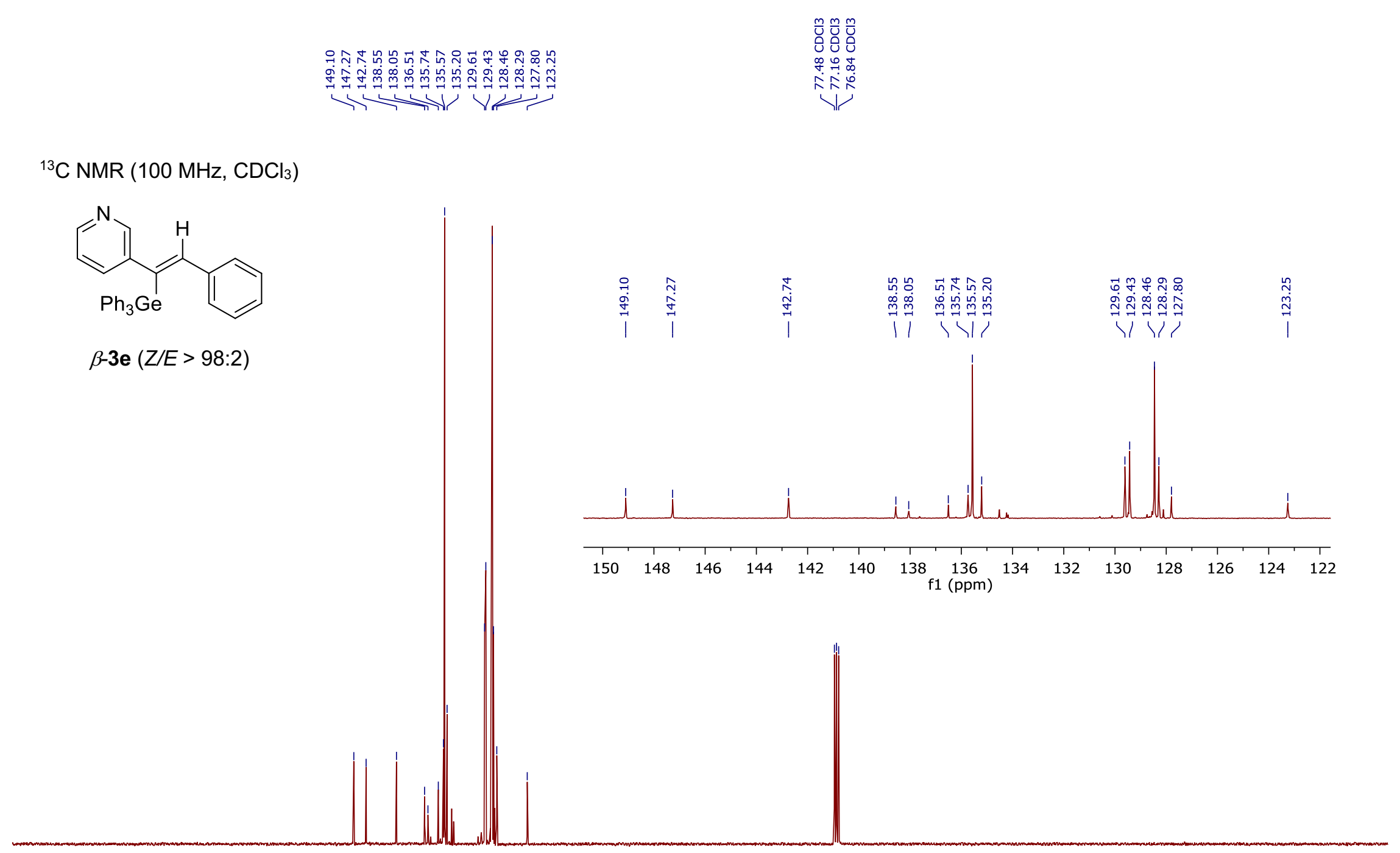

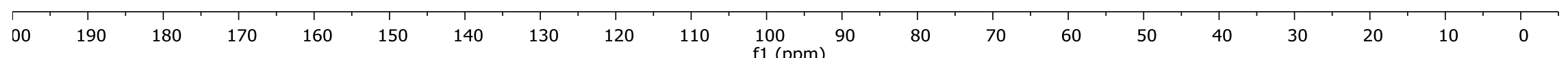




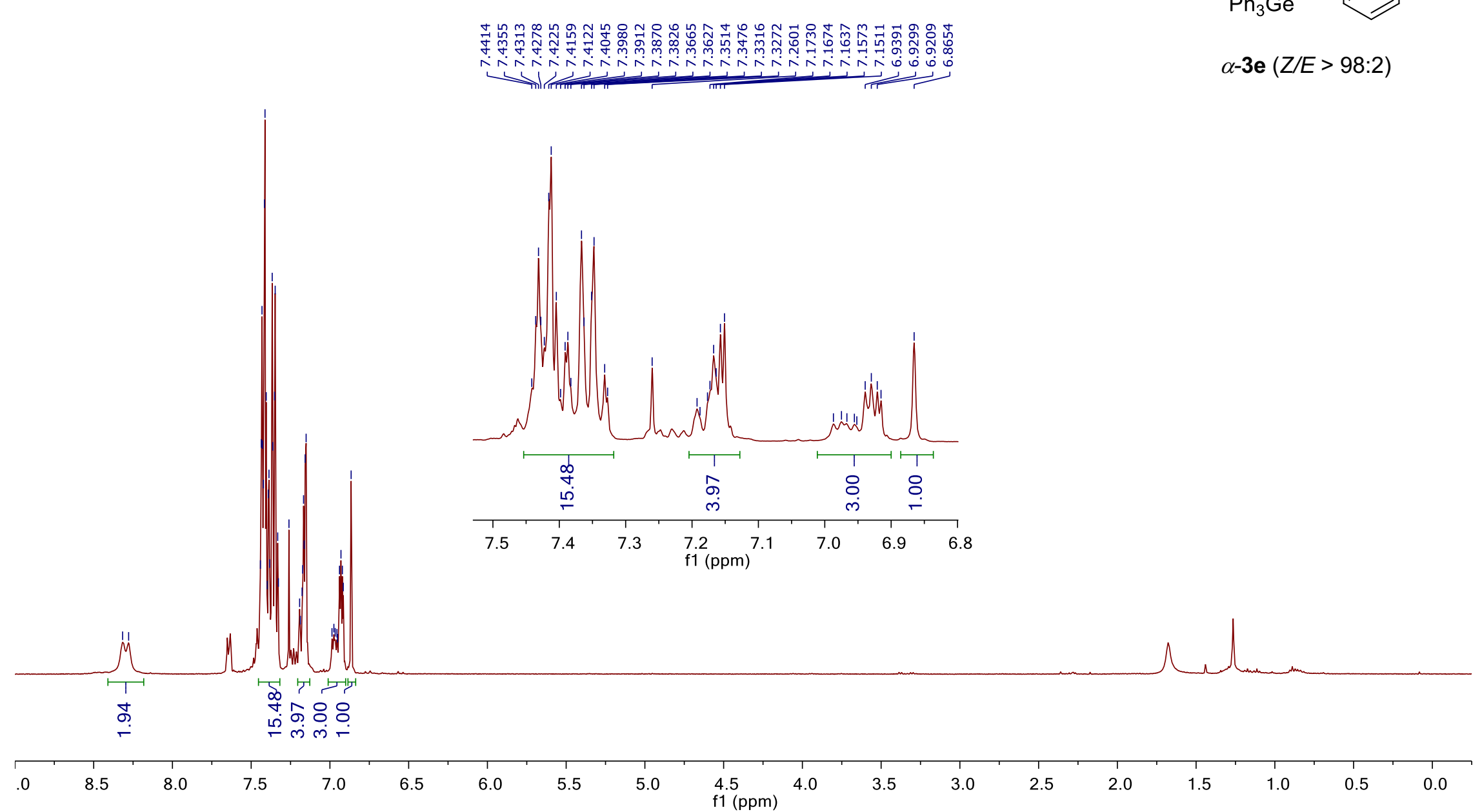

${ }^{1} \mathrm{H} \mathrm{NMR}\left(400 \mathrm{MHz}, \mathrm{CDCl}_{3}\right)$

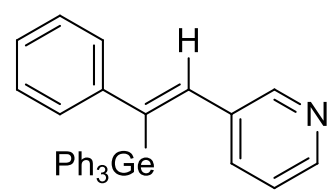

$\alpha-3 e(Z / E>98: 2)$

(ppm)

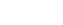




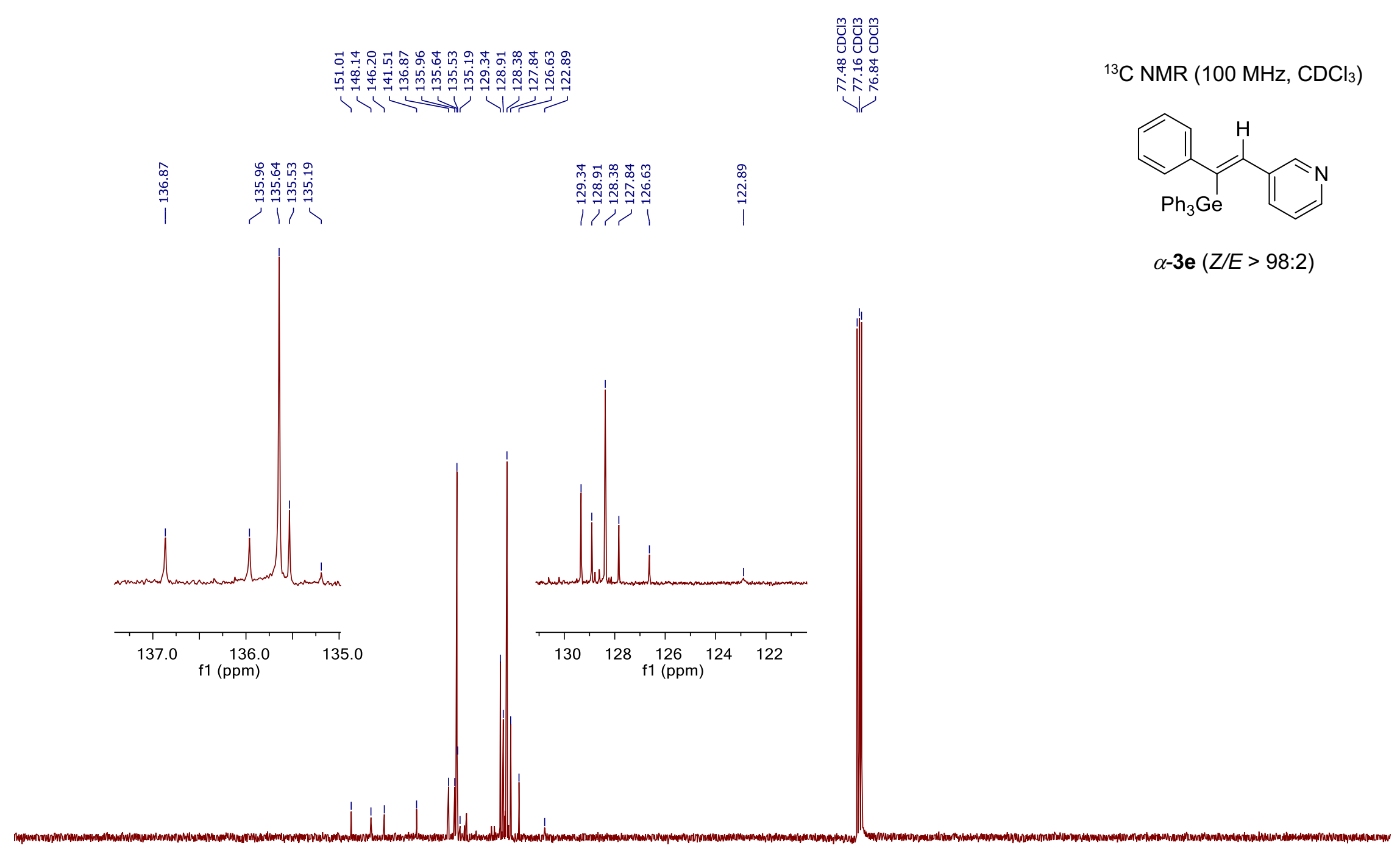

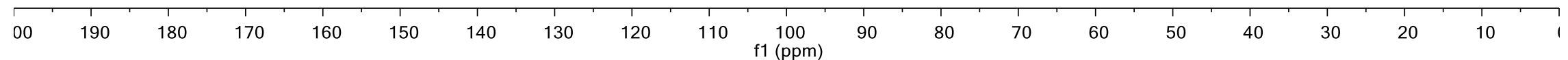


${ }^{1} \mathrm{H}$ NMR $\left(400 \mathrm{MHz}, \mathrm{CDCl}_{3}\right)$

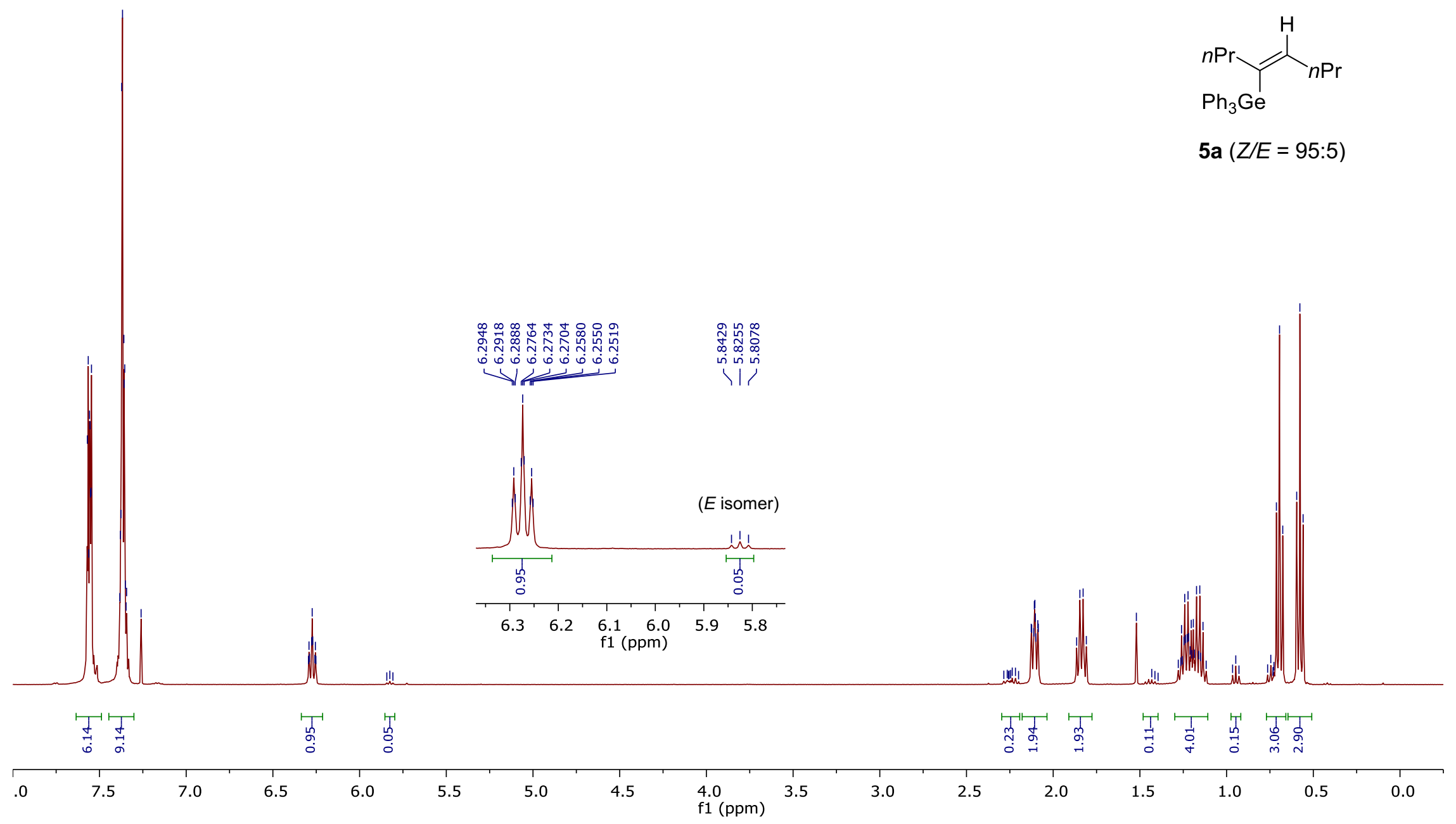




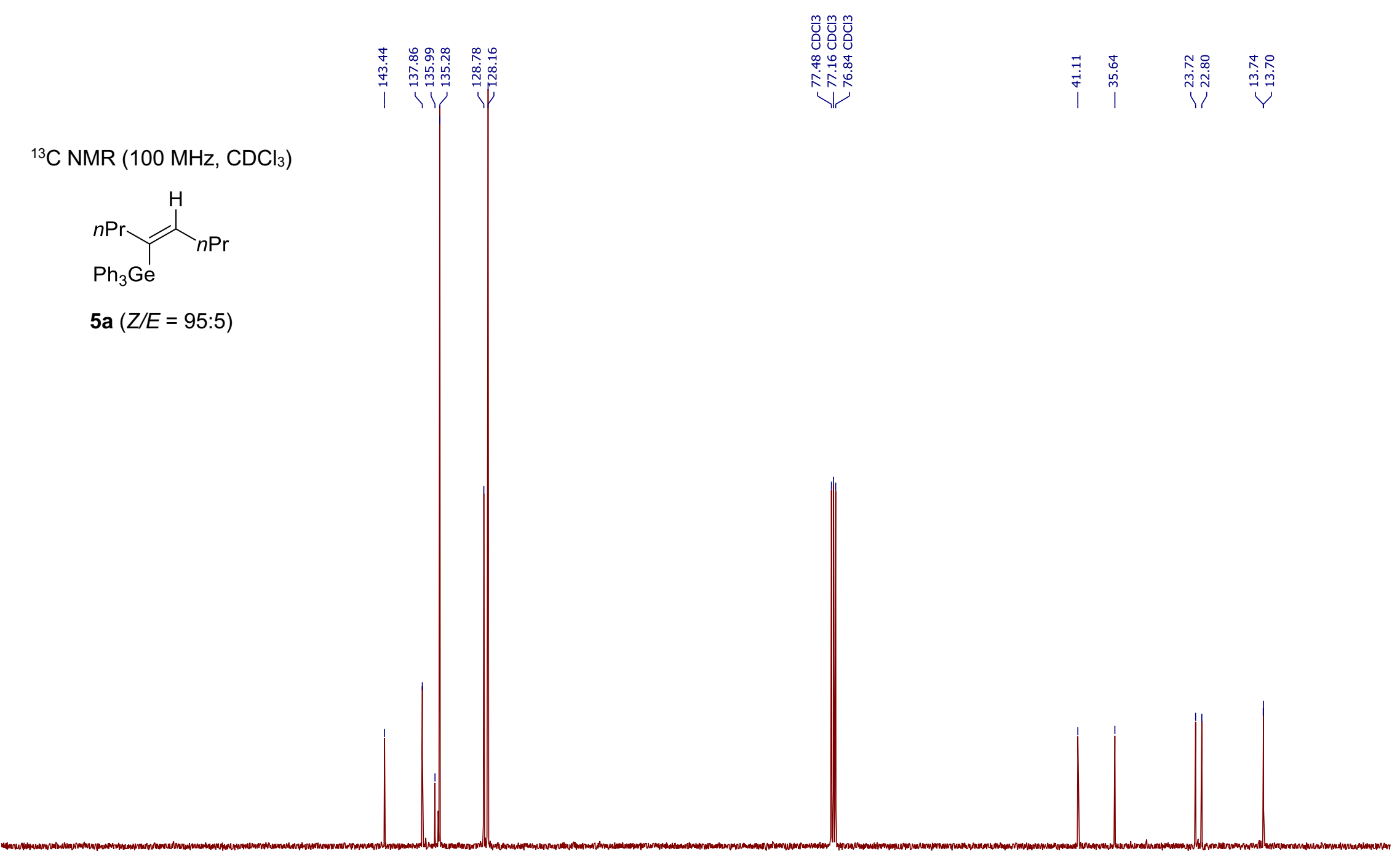

00
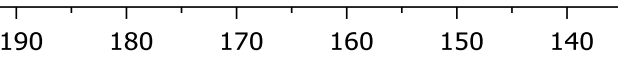

130

120

110

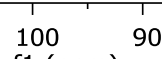

100

80

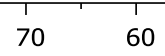




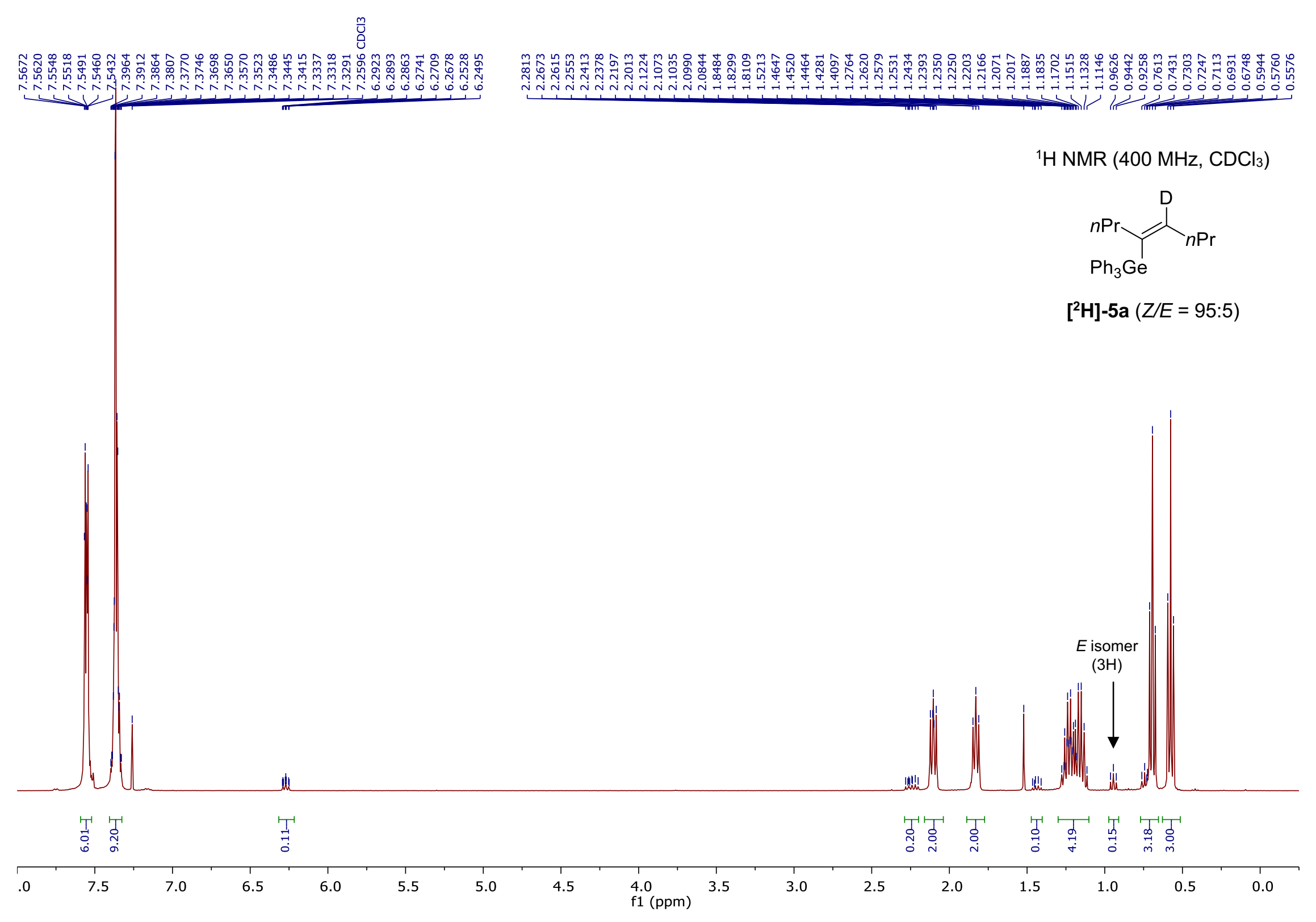




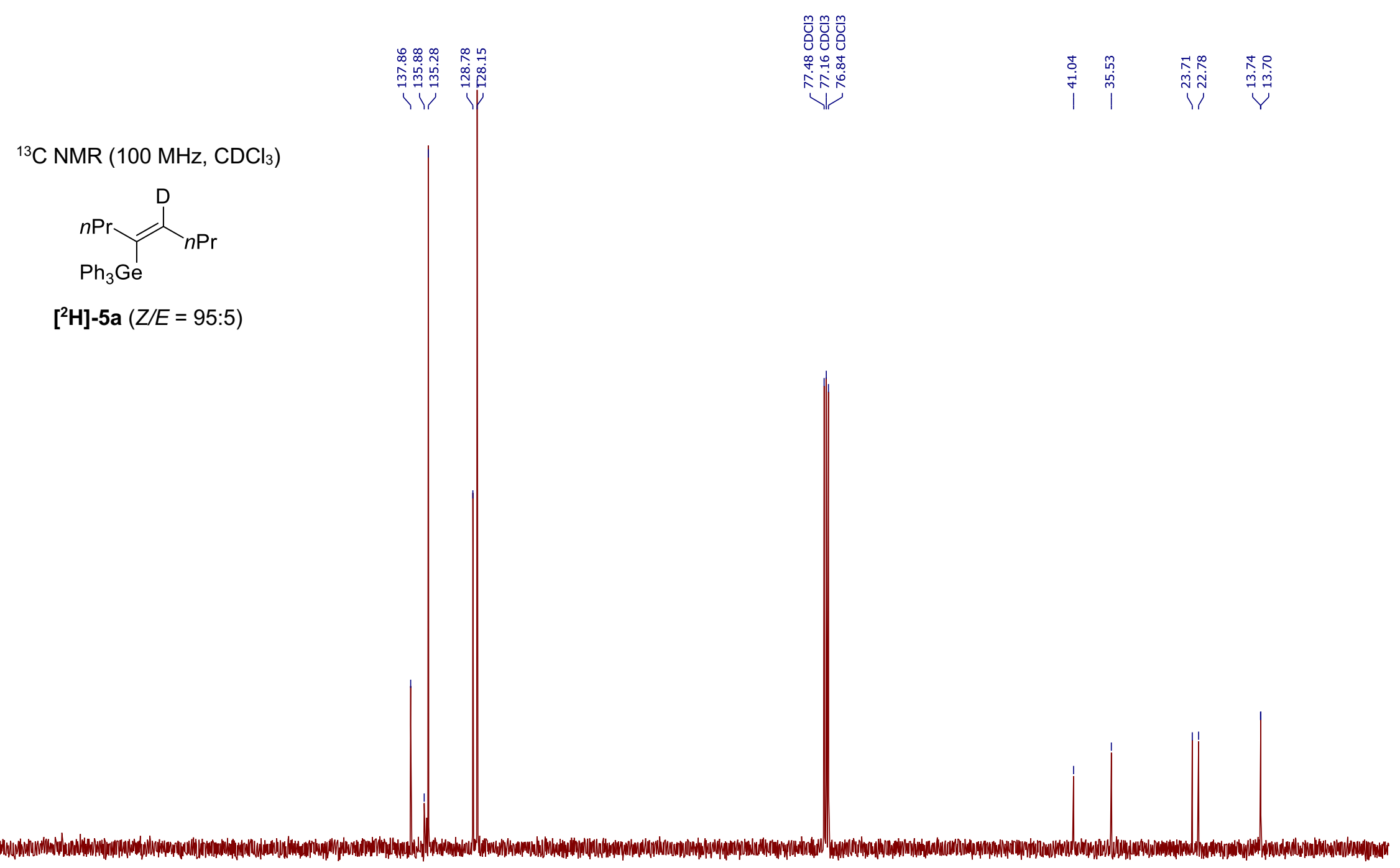

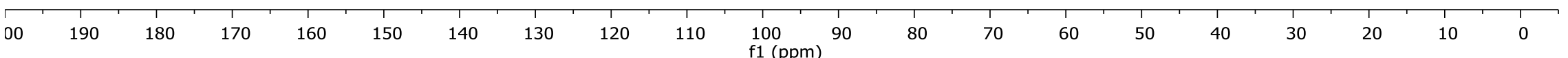


${ }^{1} \mathrm{H} \mathrm{NMR}\left(400 \mathrm{MHz}, \mathrm{CDCl}_{3}\right)$

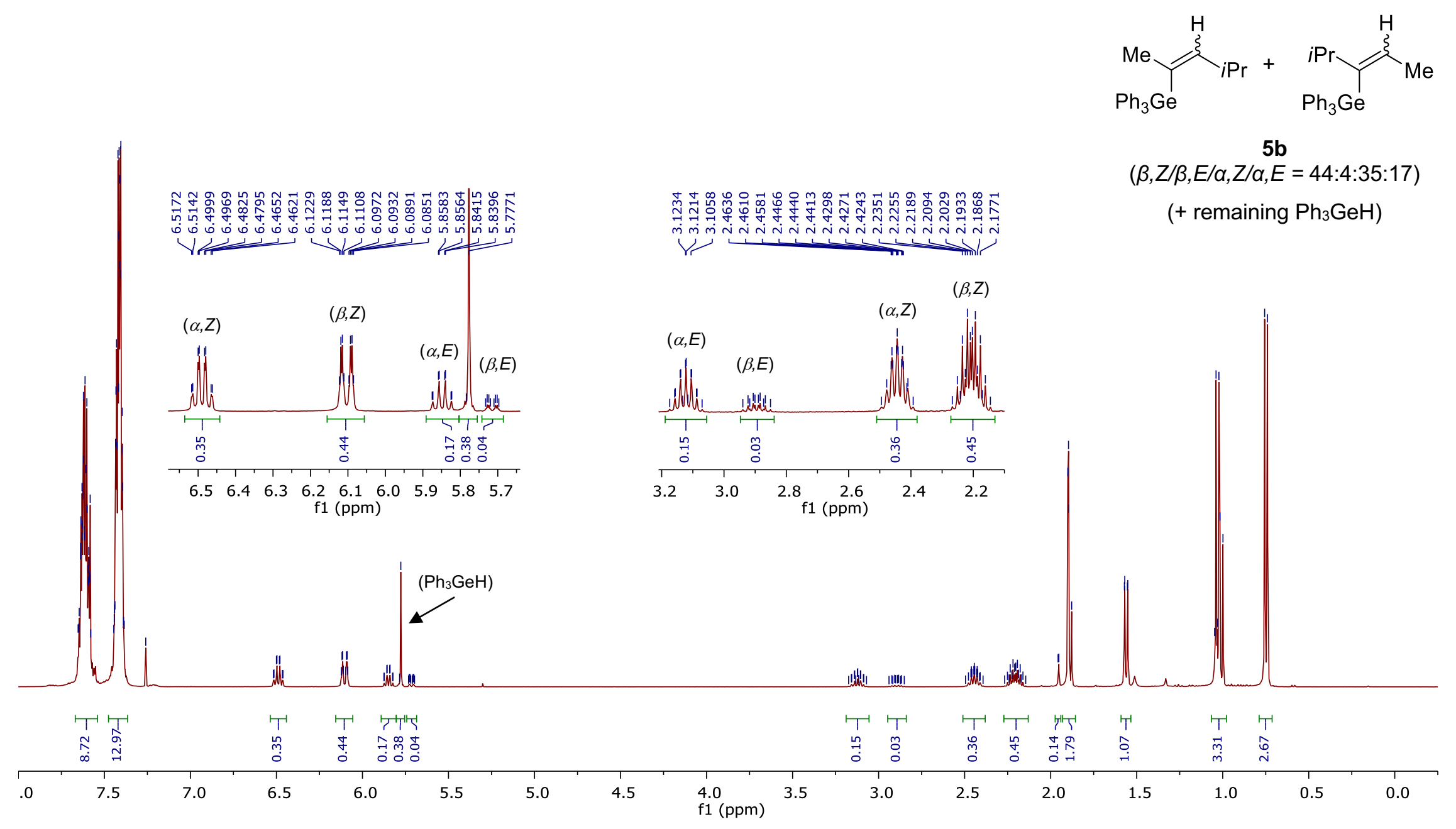




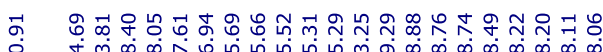

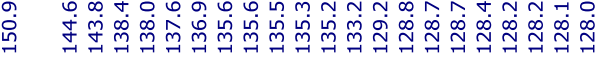

$\stackrel{m}{\mathrm{U}} \stackrel{m}{\mathrm{O}} \stackrel{m}{\mathrm{O}}$

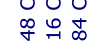

㖶

证

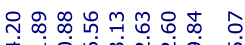
计 II। पर, ।

${ }^{13} \mathrm{C}$ NMR $\left(100 \mathrm{MHz}, \mathrm{CDCl}_{3}\right)$

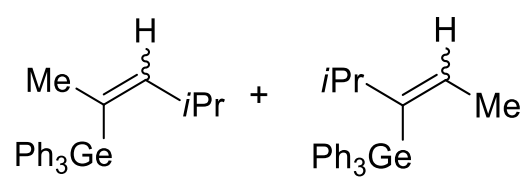
$5 \mathrm{~b}$

$(\beta, Z / \beta, E / \alpha, Z / \alpha, E=44: 4: 35: 17)$ (+ remaining $\mathrm{Ph}_{3} \mathrm{GeH}$ )

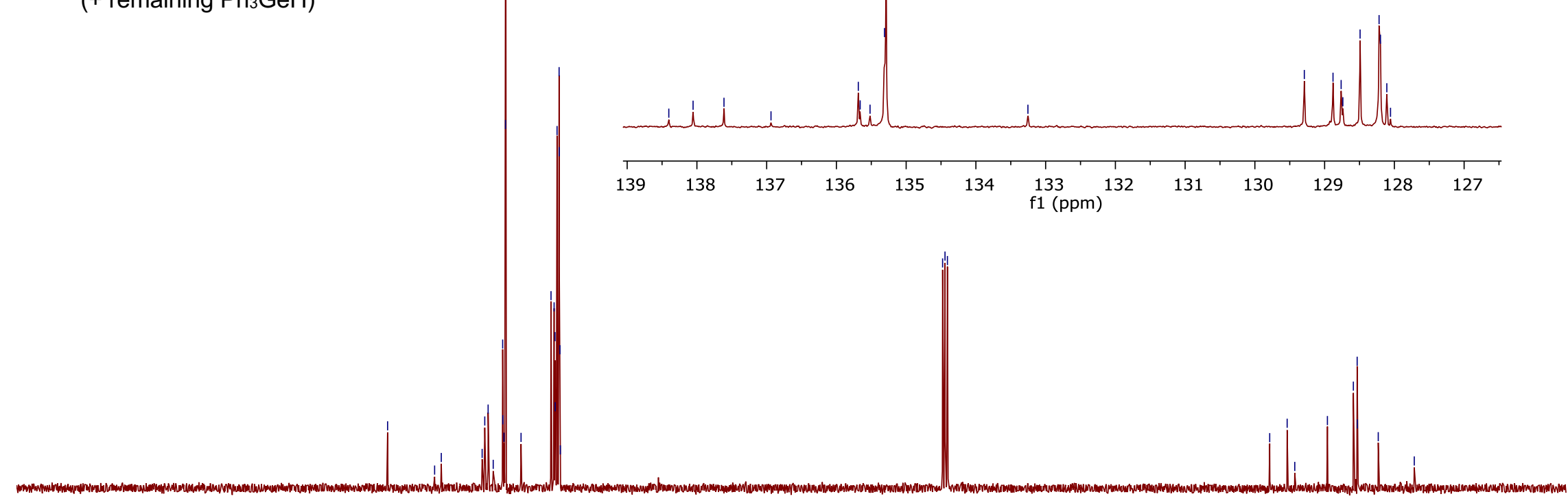

00 


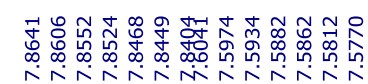

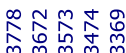

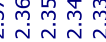

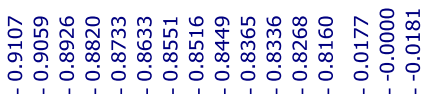

${ }^{1} \mathrm{H} \mathrm{NMR}\left(400 \mathrm{MHz}, \mathrm{CDCl}_{3}\right)$

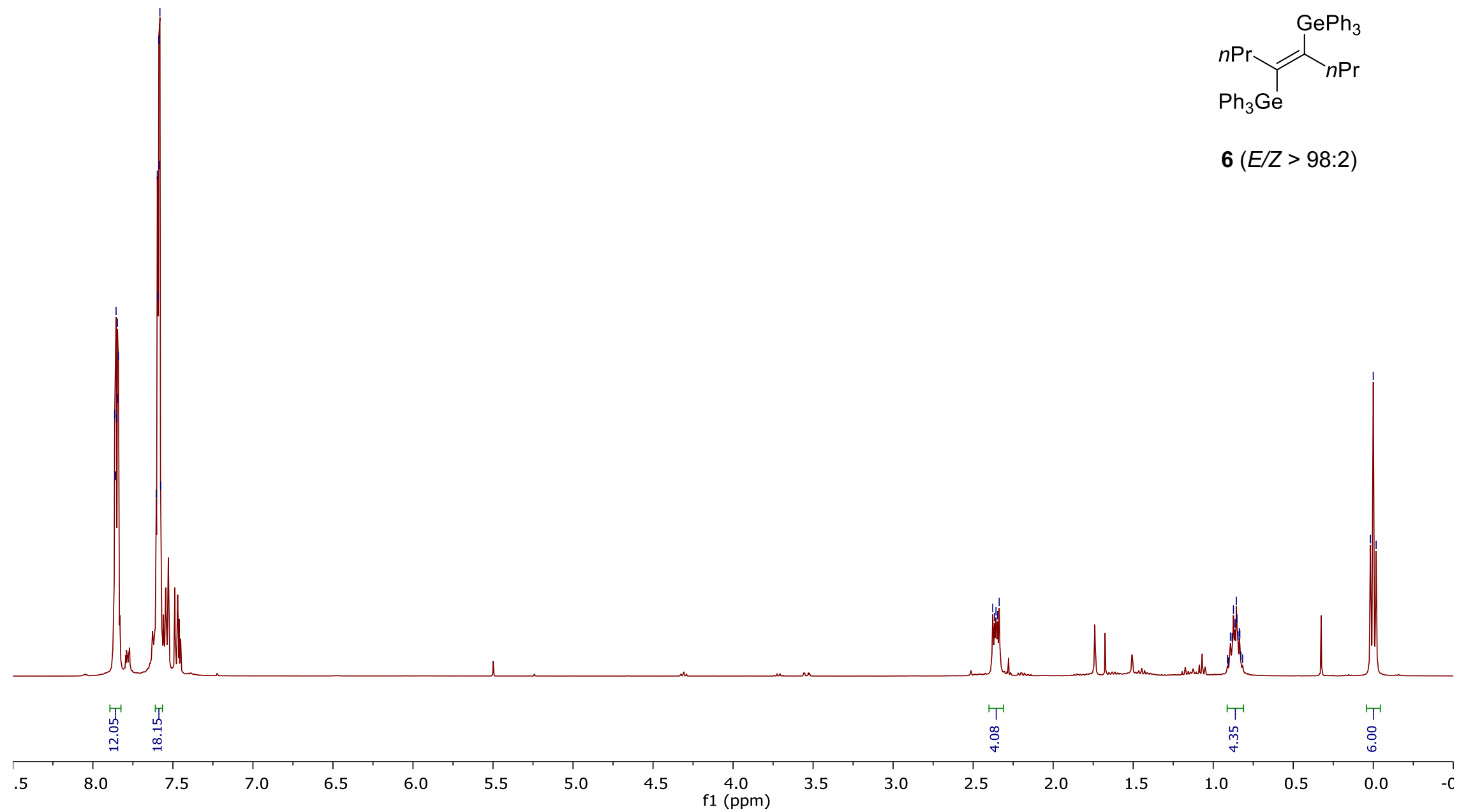




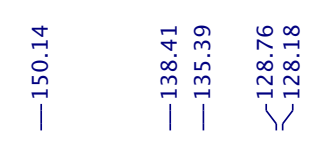

o 60

全芹

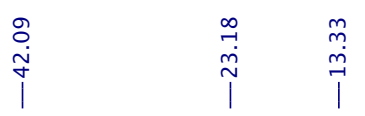

${ }^{13} \mathrm{C}$ NMR $\left(100 \mathrm{MHz}, \mathrm{CDCl}_{3}\right)$

$$
6(E / Z>98: 2)
$$

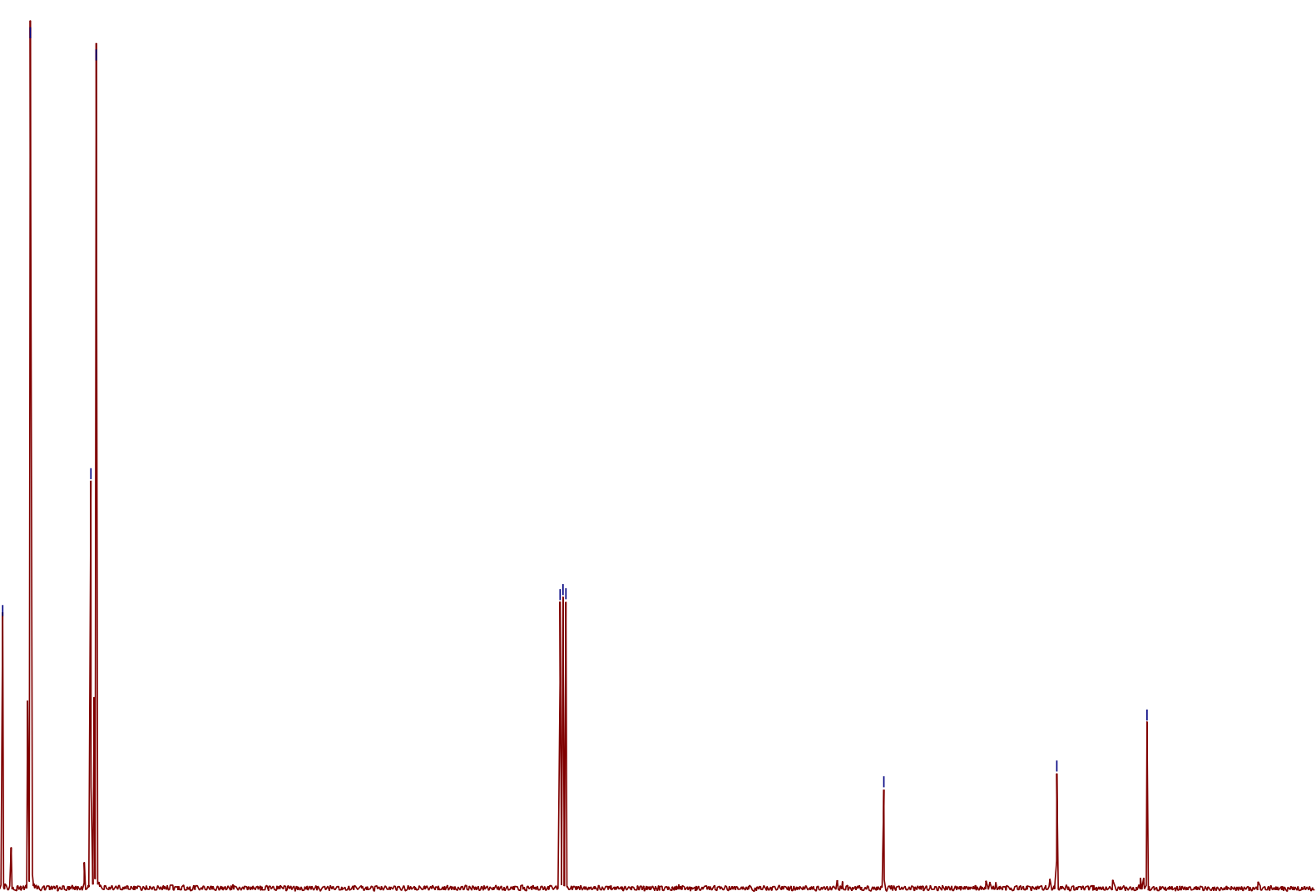

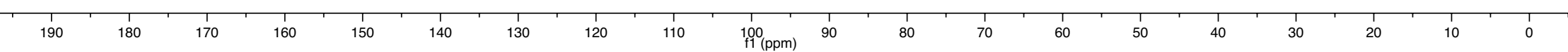




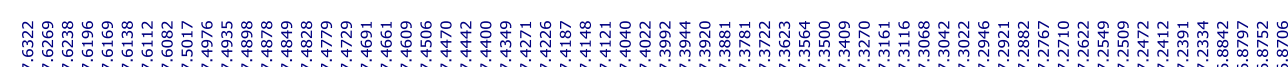

บับ

${ }^{1} \mathrm{H}$ NMR (400 MHz, $\left.\mathrm{CDCl}_{3}\right)$

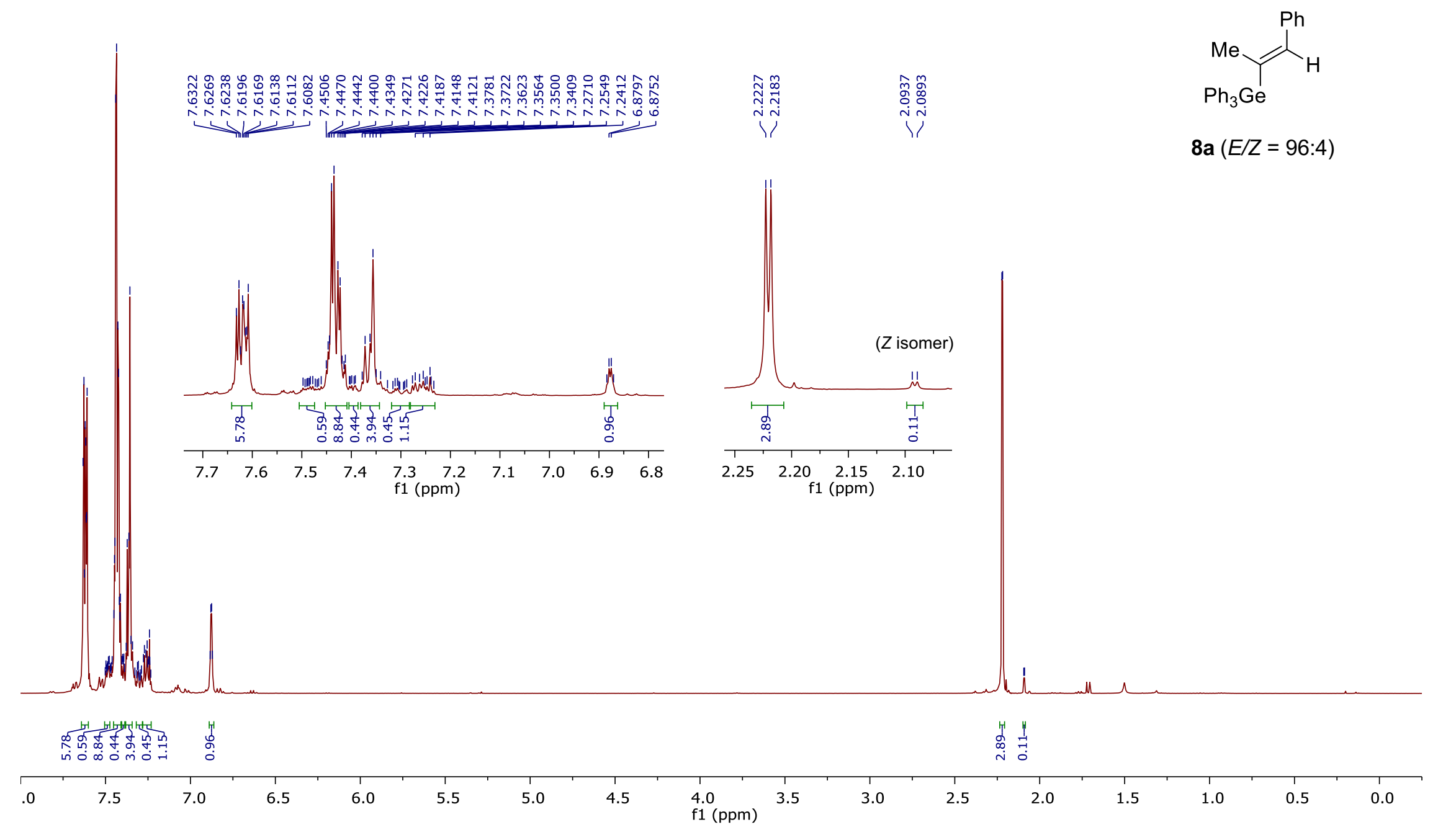


둥ㅇํ우요

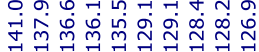

रोiा $m$
$\mathrm{O}$
$\mathrm{O}$
$\mathrm{O}$
0

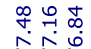

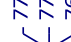

${ }^{13} \mathrm{C} \mathrm{NMR}\left(100 \mathrm{MHz}, \mathrm{CDCl}_{3}\right)$

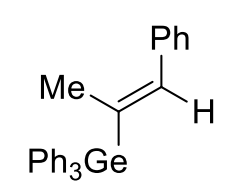

8a $(E / Z=96: 4)$

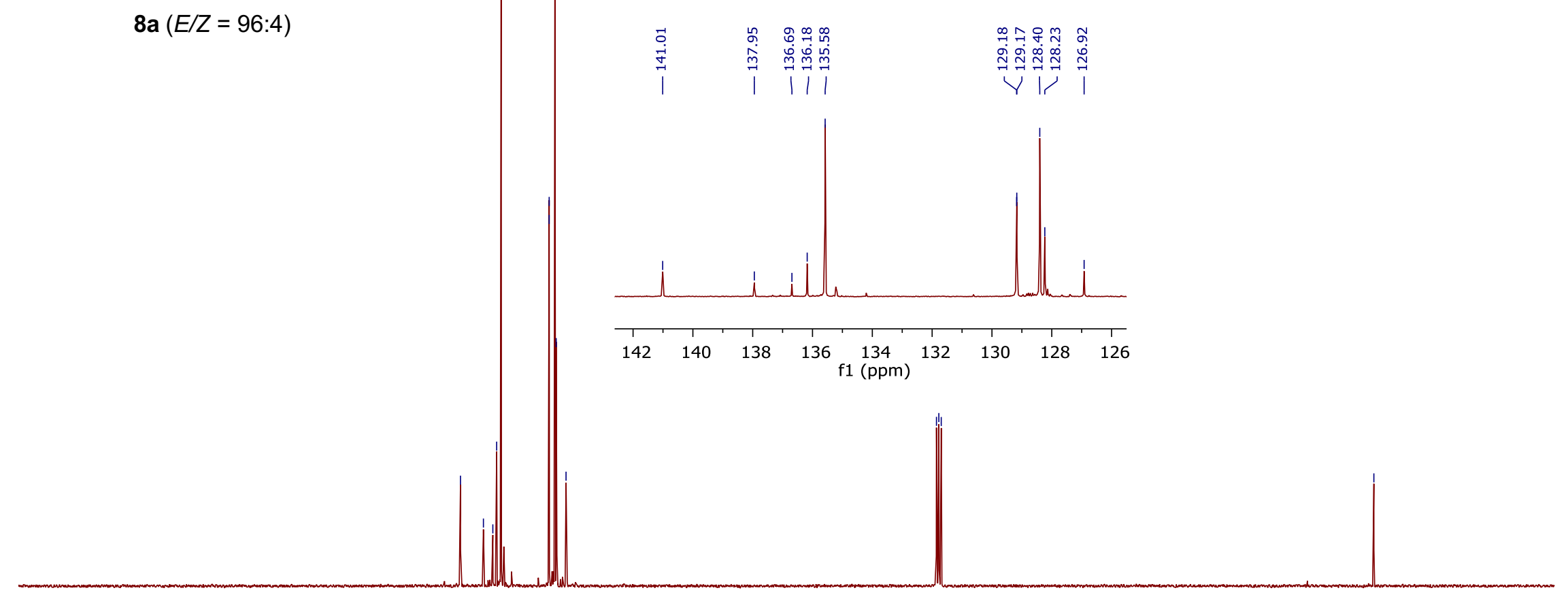




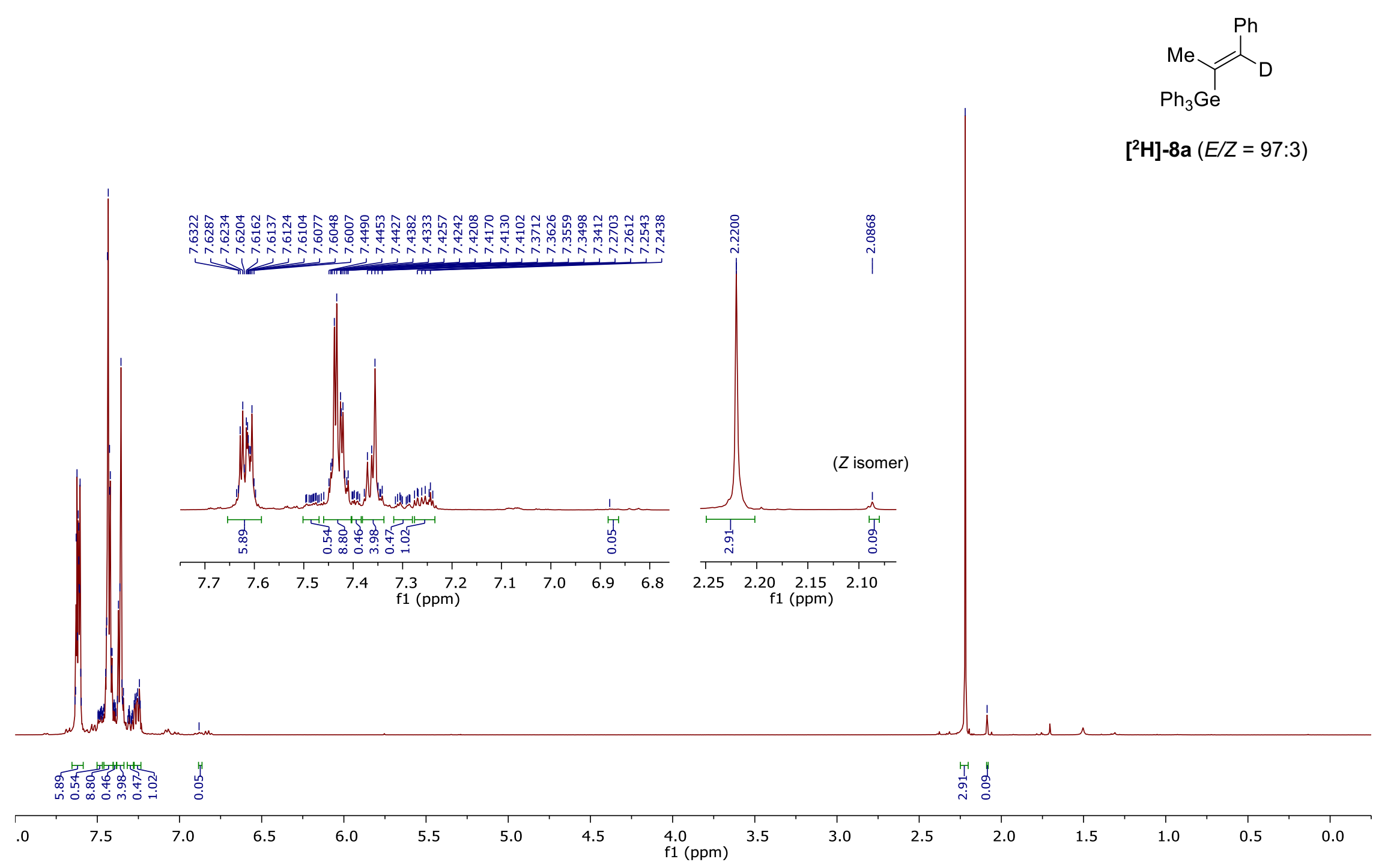




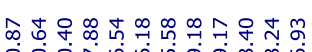

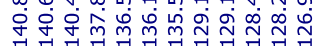

垈

商商商

象是

被

$\stackrel{\circ}{\circ}$

$\left.{ }^{13} \mathrm{C} \mathrm{NMR} \mathrm{(100} \mathrm{MHz,} \mathrm{CDCl}_{3}\right)$

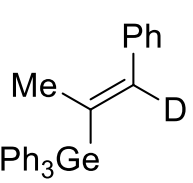

$\left.{ }^{2} \mathbf{H}\right]-8 \mathbf{a}(E / Z=97: 3)$

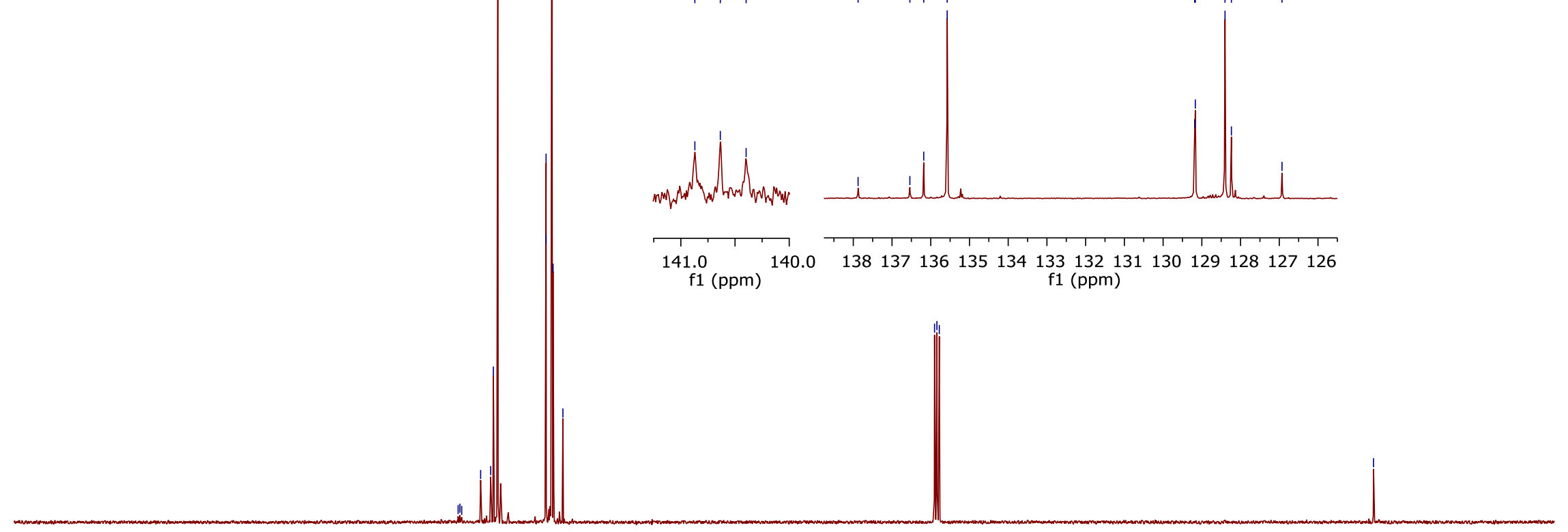

190

180

170

$160 \quad 150$

140

130

120

110 
${ }^{1} \mathrm{H}$ NMR $\left(400 \mathrm{MHz}, \mathrm{CDCl}_{3}\right)$

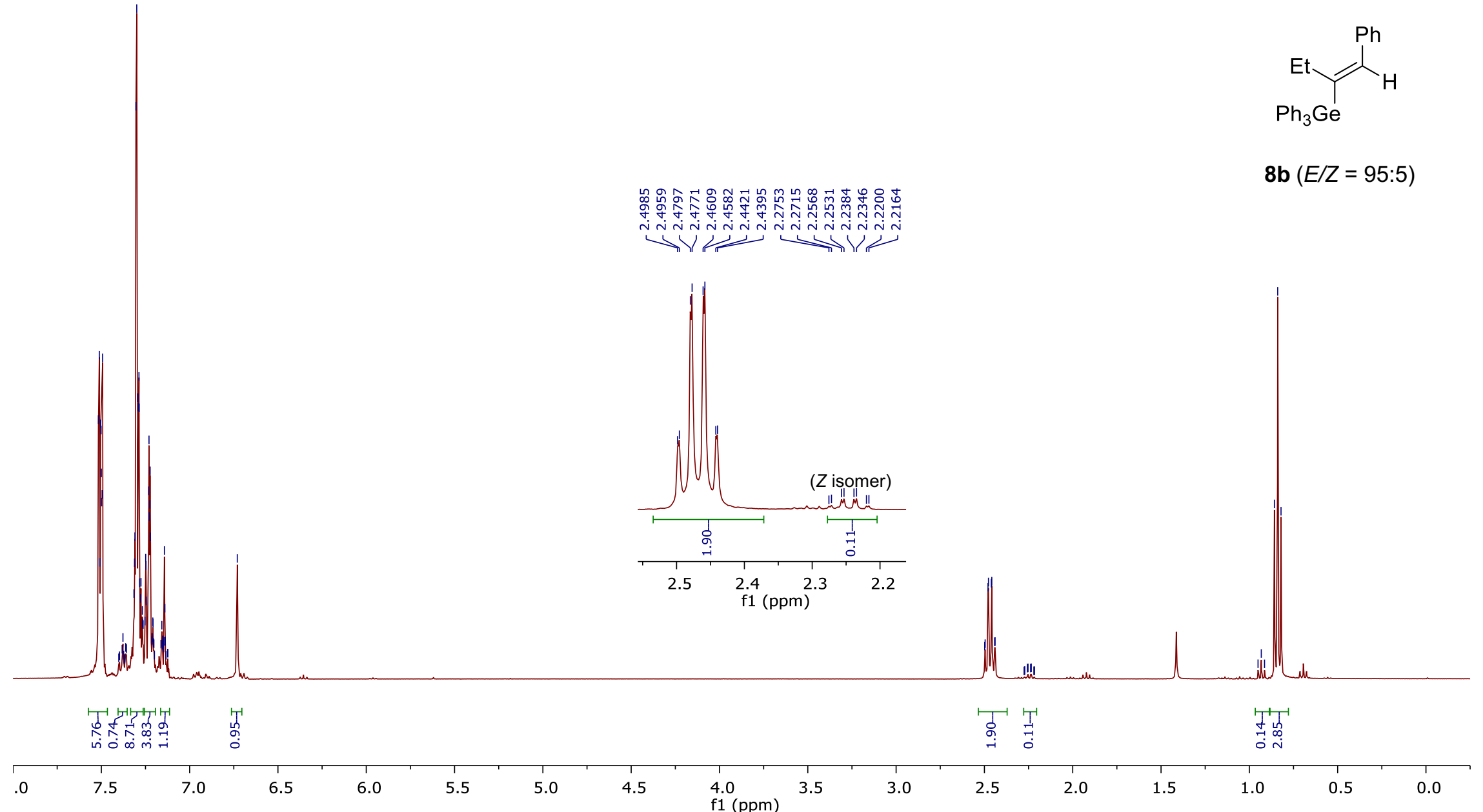




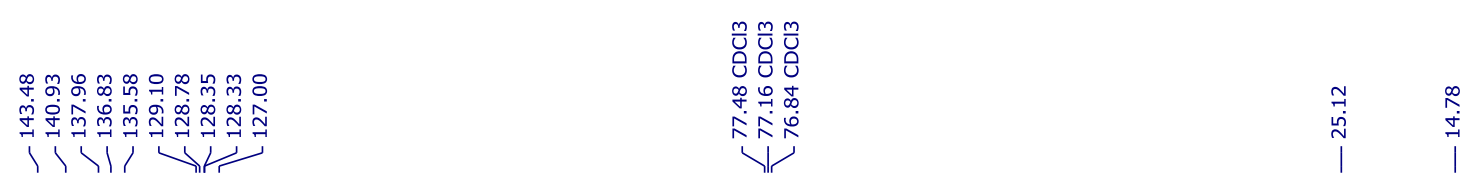

${ }^{13} \mathrm{C} \mathrm{NMR}\left(100 \mathrm{MHz}, \mathrm{CDCl}_{3}\right)$

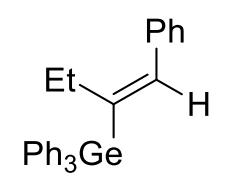

$\mathbf{8 b}(E / Z=95: 5)$

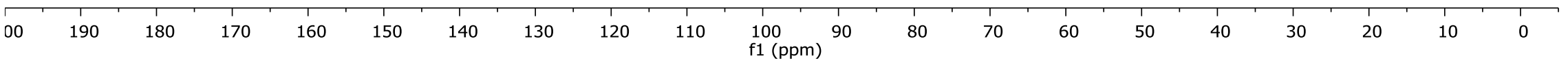




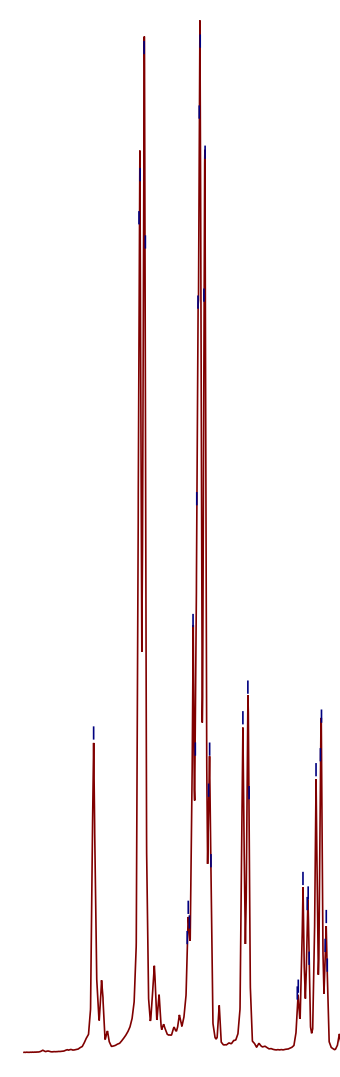

年

${ }^{1} \mathrm{H}$ NMR $\left(400 \mathrm{MHz}, \mathrm{CDCl}_{3}\right)$

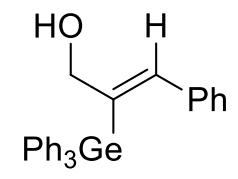

13a $(Z / E>98: 2)$

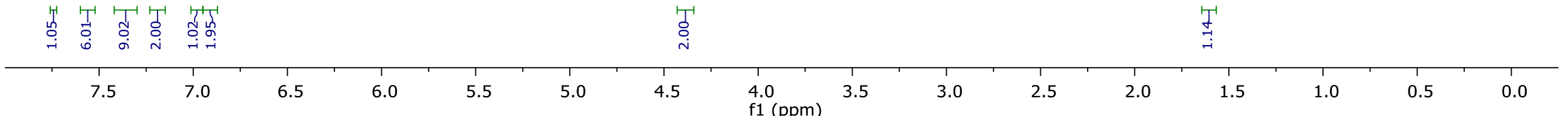




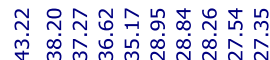

I पil/ V/1

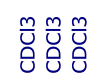

क⿻丷木

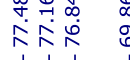

${ }^{13} \mathrm{C} \mathrm{NMR}\left(100 \mathrm{MHz}, \mathrm{CDCl}_{3}\right)$

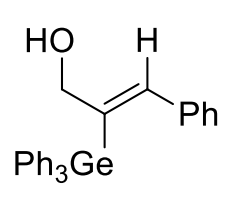

13a $(Z / E>98: 2)$

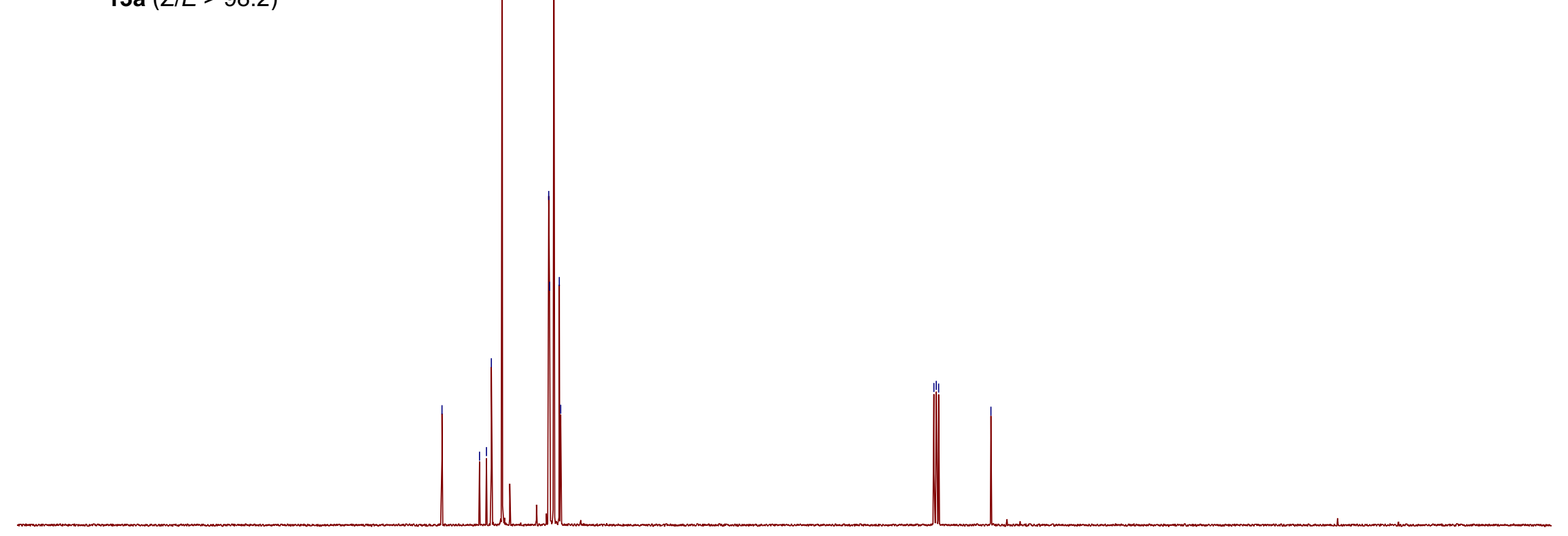




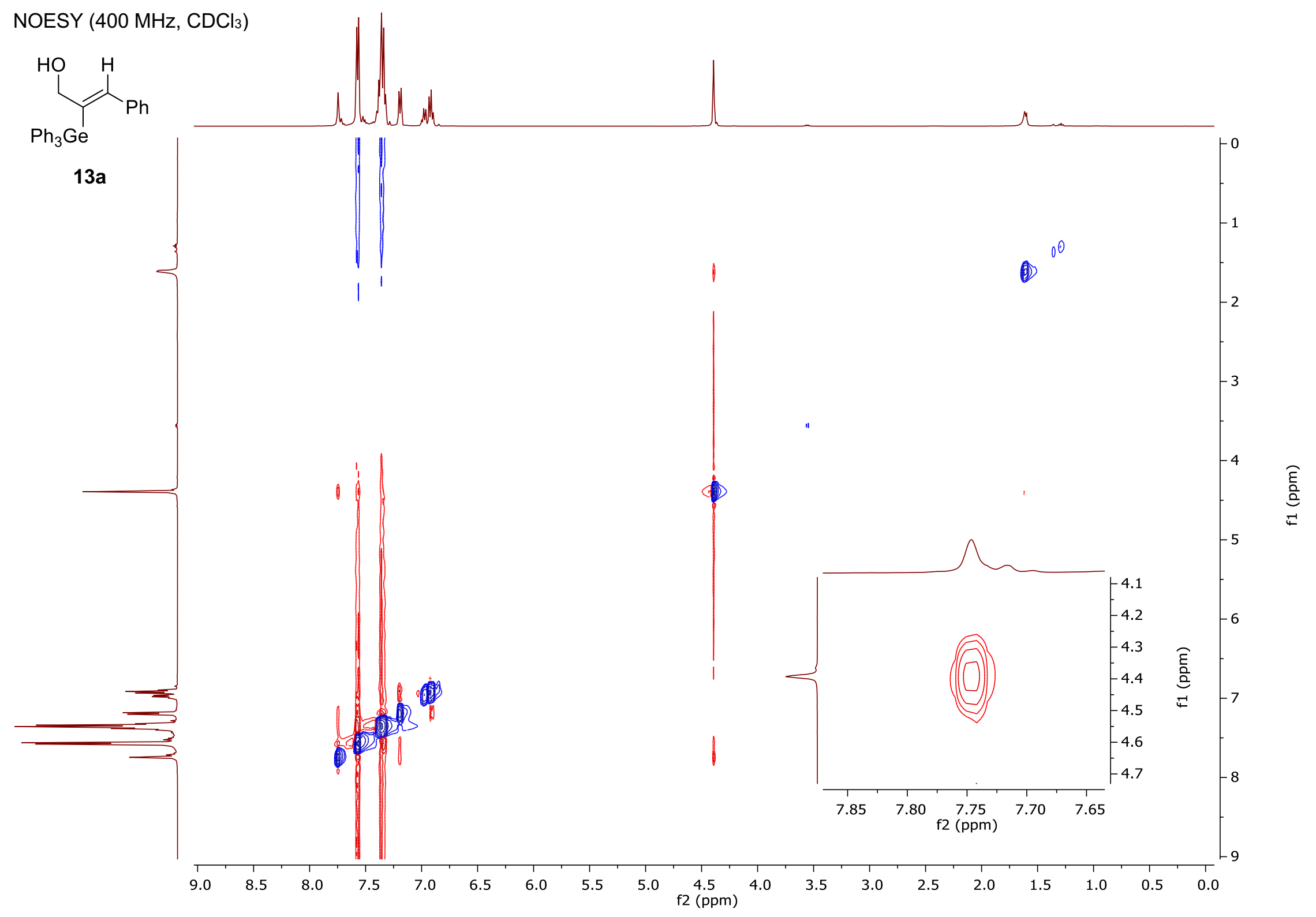




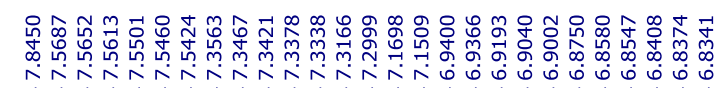

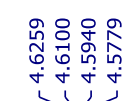

|

${ }^{1} \mathrm{H}$ NMR $\left(400 \mathrm{MHz}, \mathrm{CDCl}_{3}\right)$

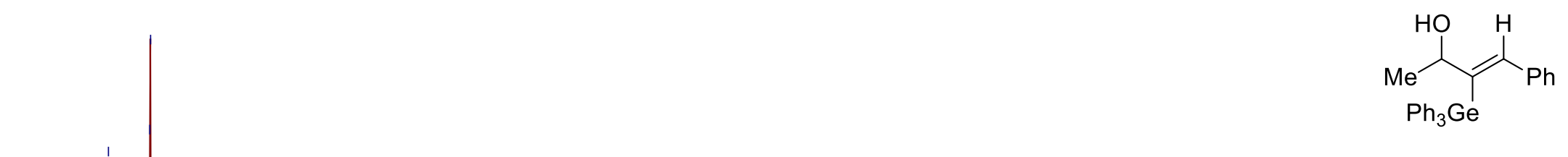

13b $(Z / E>98: 2)$

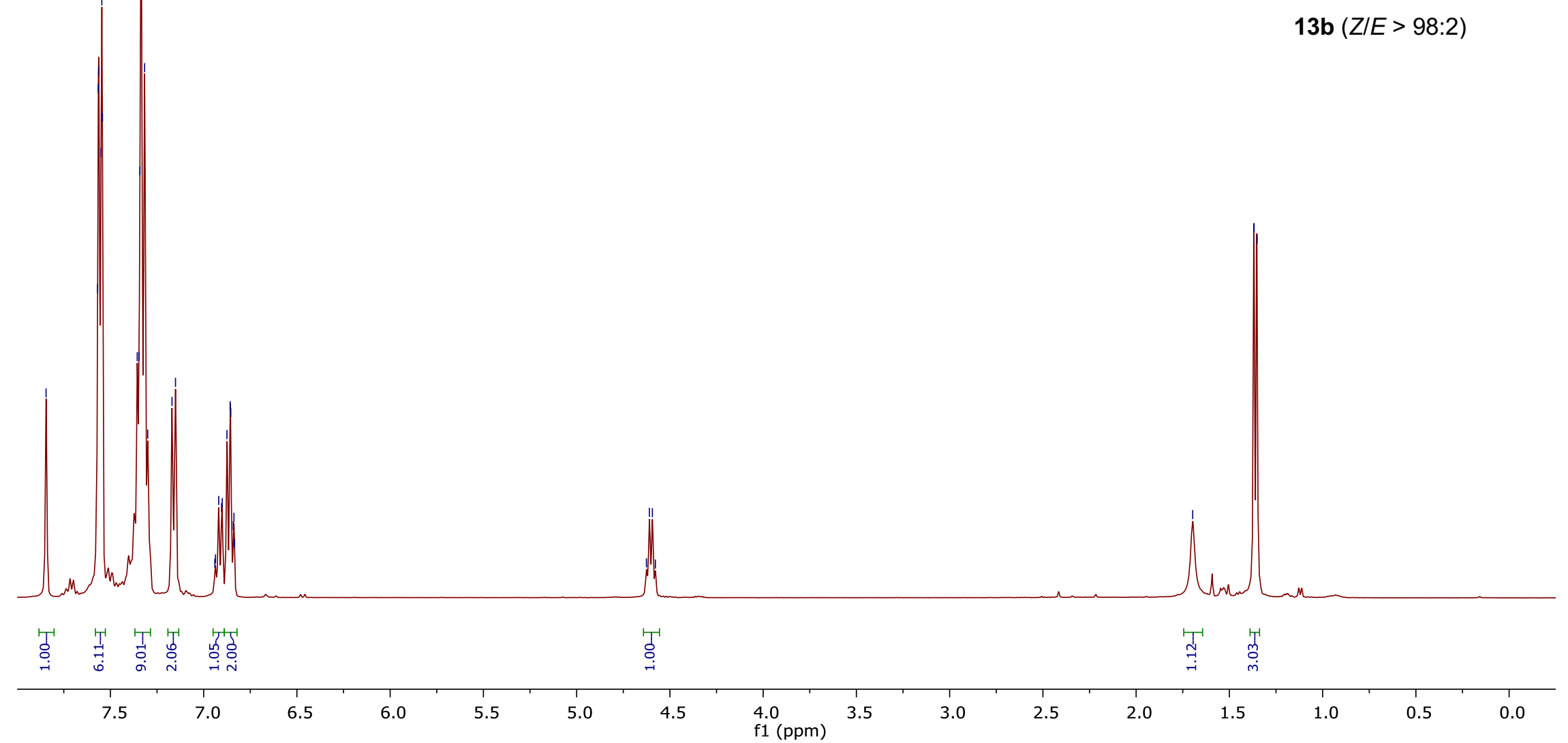




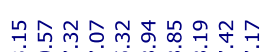

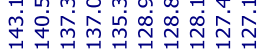

।रे।

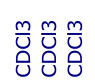

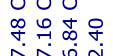

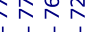

N)

${ }^{13} \mathrm{C}$ NMR $\left(100 \mathrm{MHz}, \mathrm{CDCl}_{3}\right)$

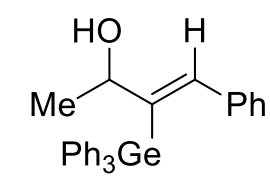

13b $(Z / E>98: 2)$
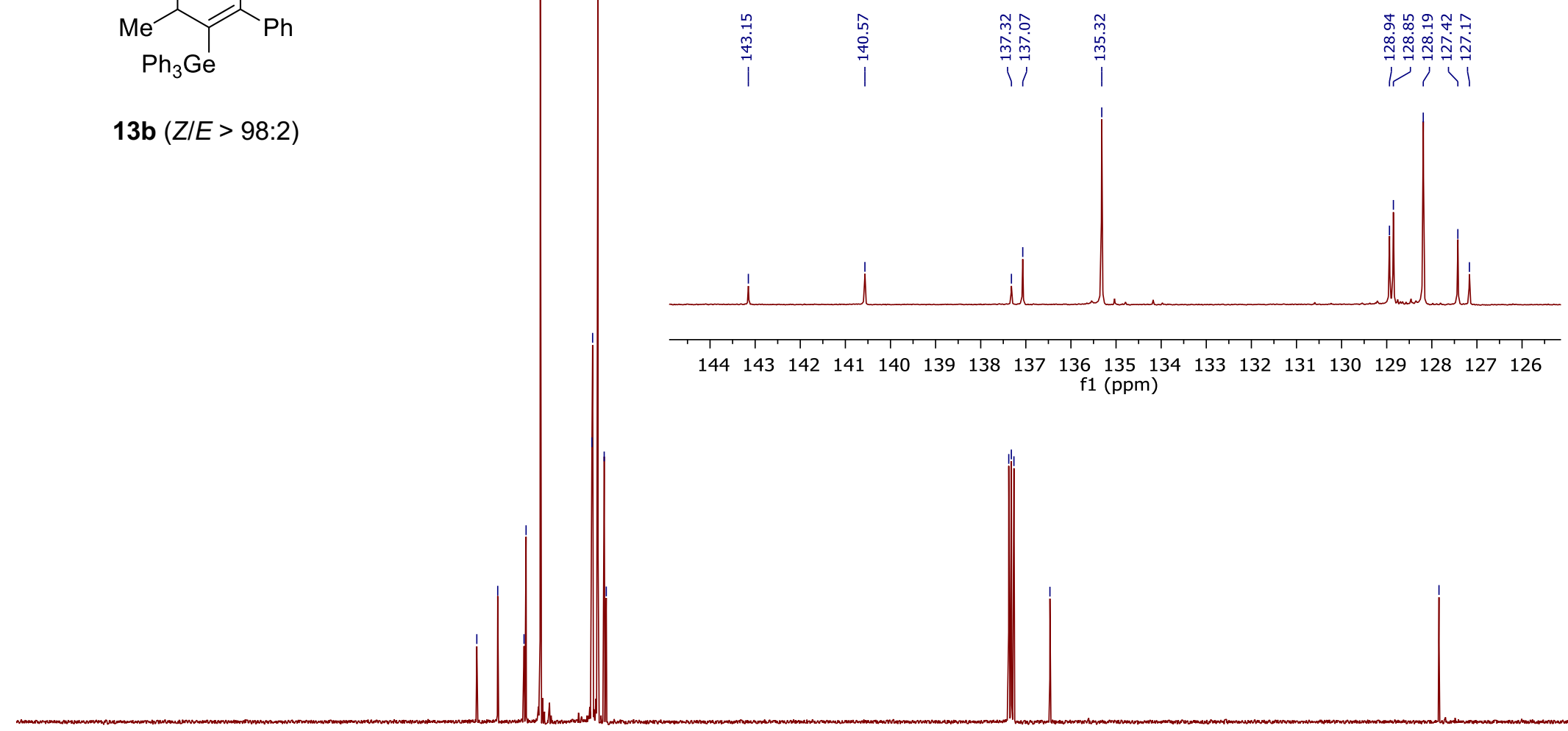

$\begin{array}{lllllllllllllllllll}144 & 143 & 142 & 141 & 140 & 139 & 138 & 137 & 136 & 135 & 134 & 133 & 132 & 131 & 130 & 129 & 128 & 127 & 126\end{array}$ f1 (ppm) 


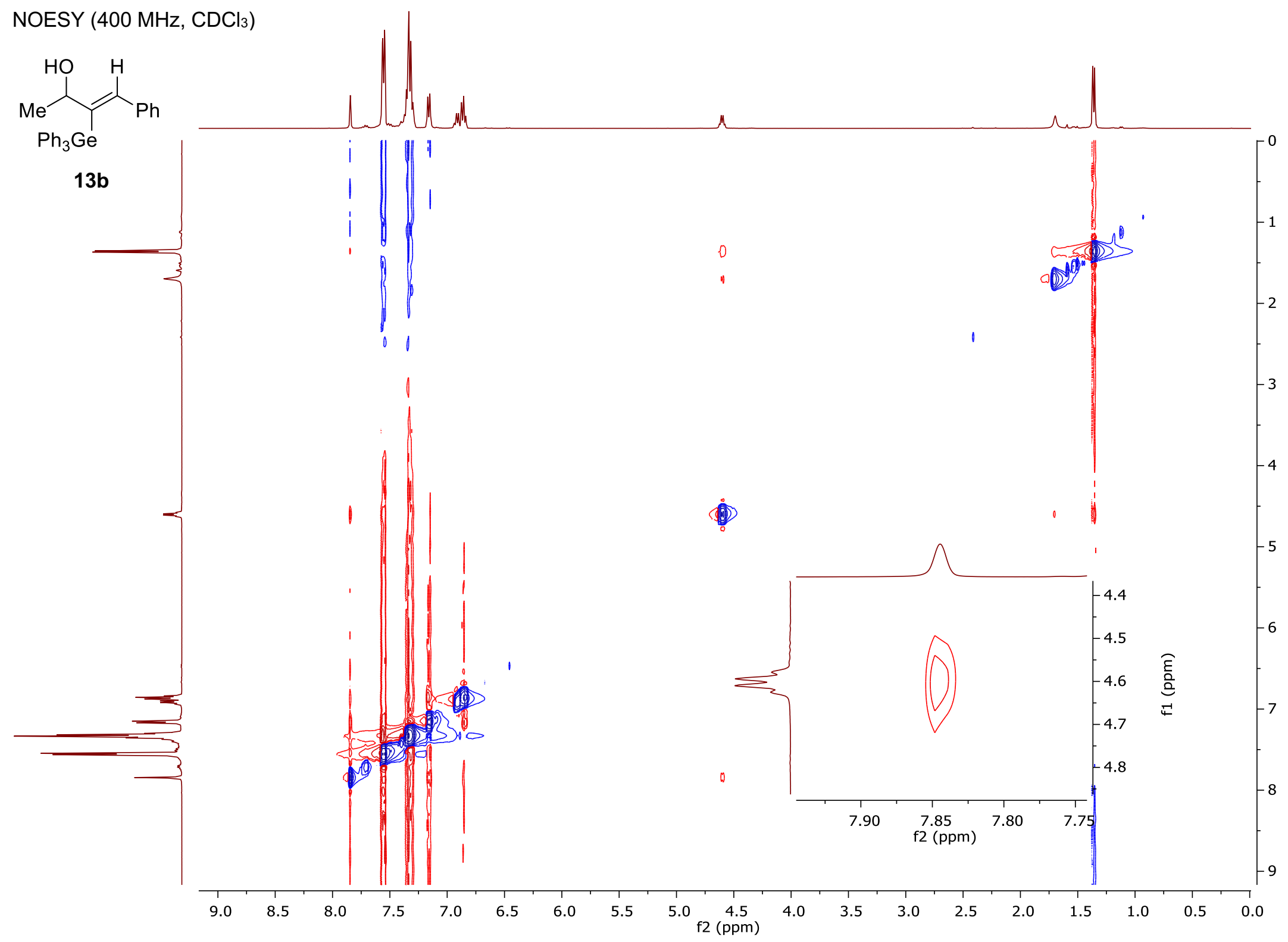

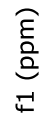




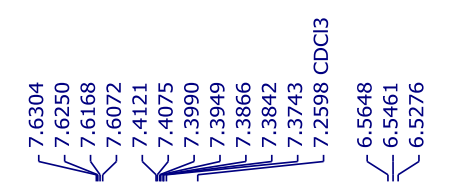

${ }^{1} \mathrm{H} \mathrm{NMR}\left(400 \mathrm{MHz}, \mathrm{CDCl}_{3}\right)$

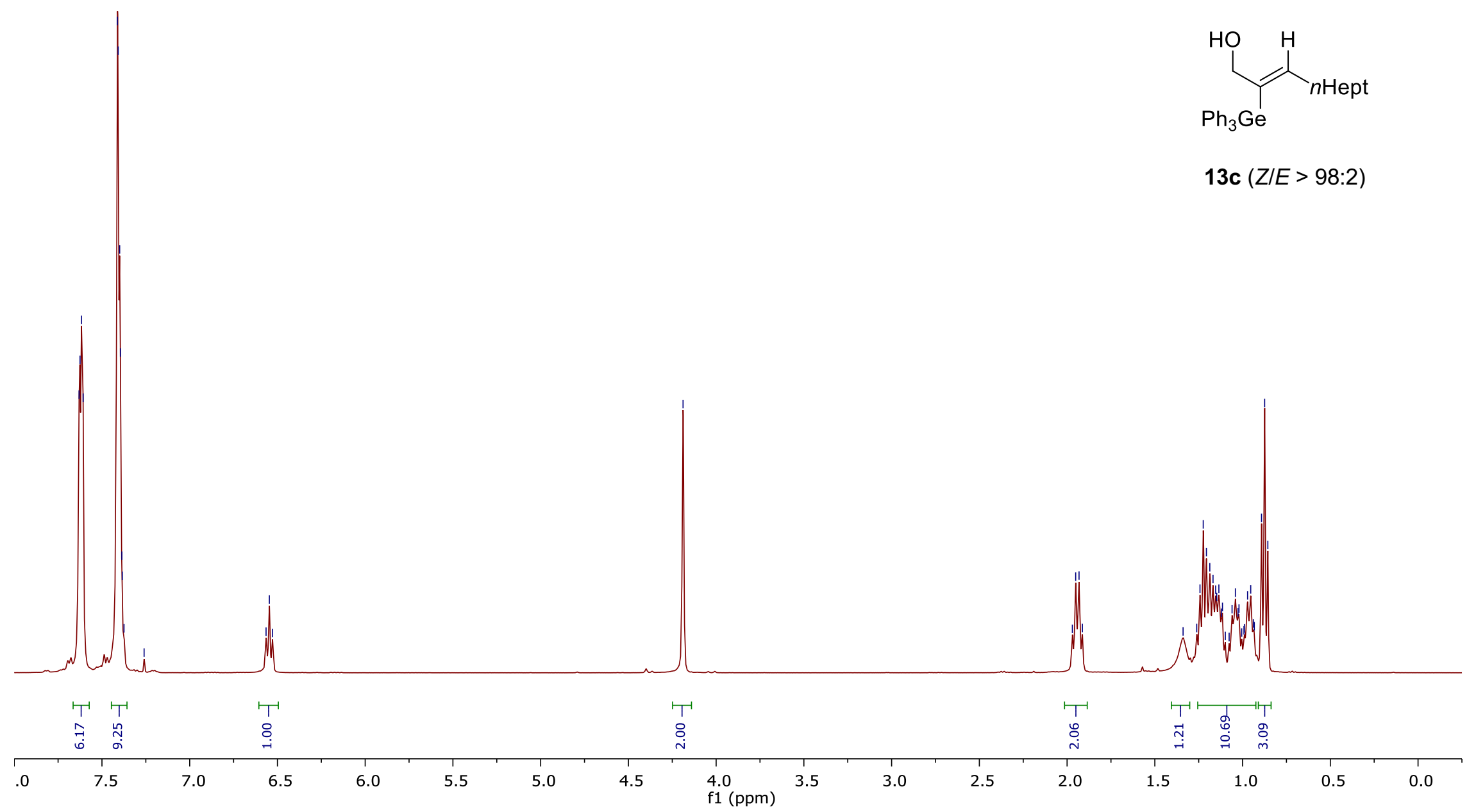




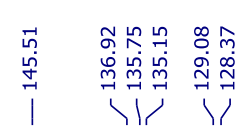

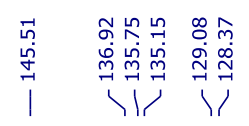

${ }^{13} \mathrm{C} \mathrm{NMR}\left(100 \mathrm{MHz}, \mathrm{CDCl}_{3}\right)$

13c $(Z / E>98: 2)$

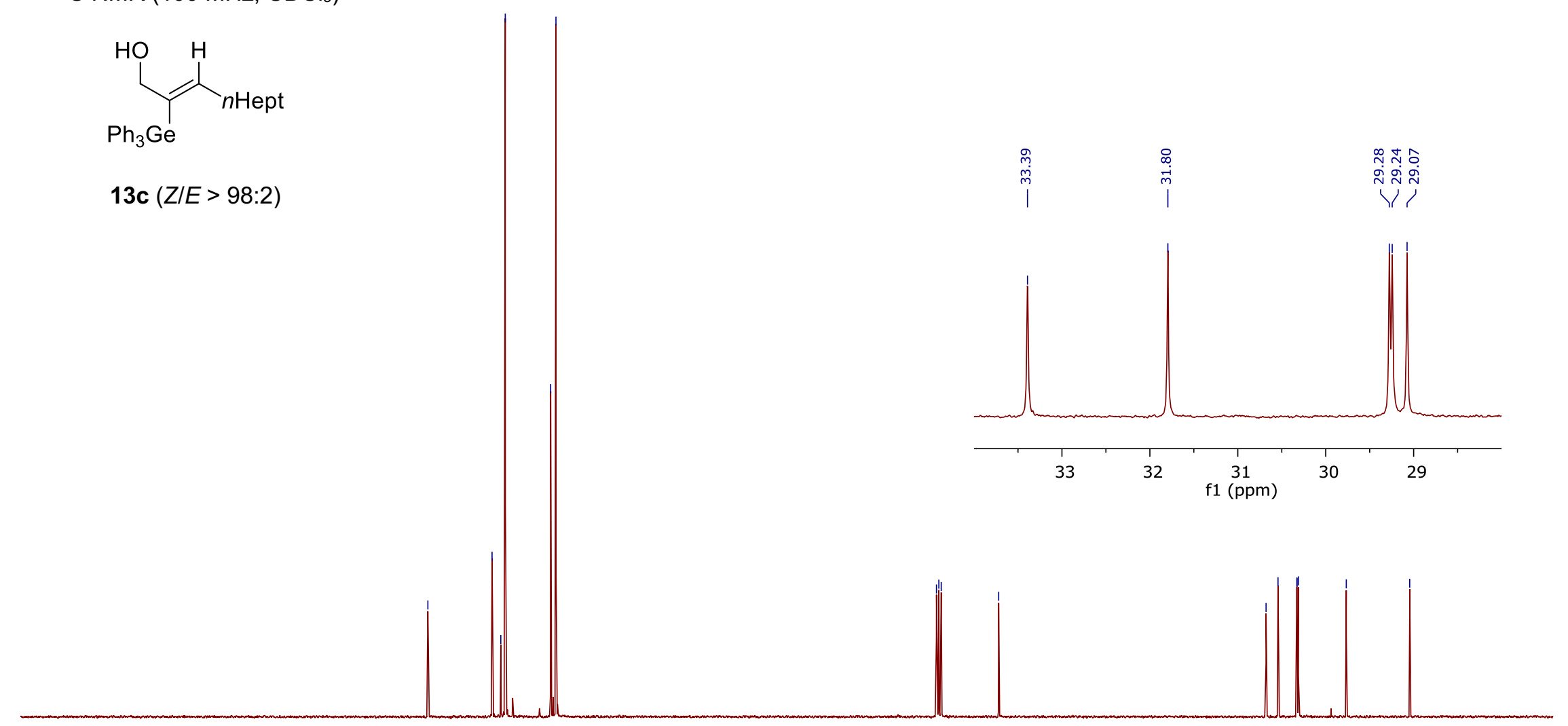

ming

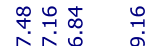

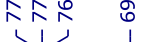

20 


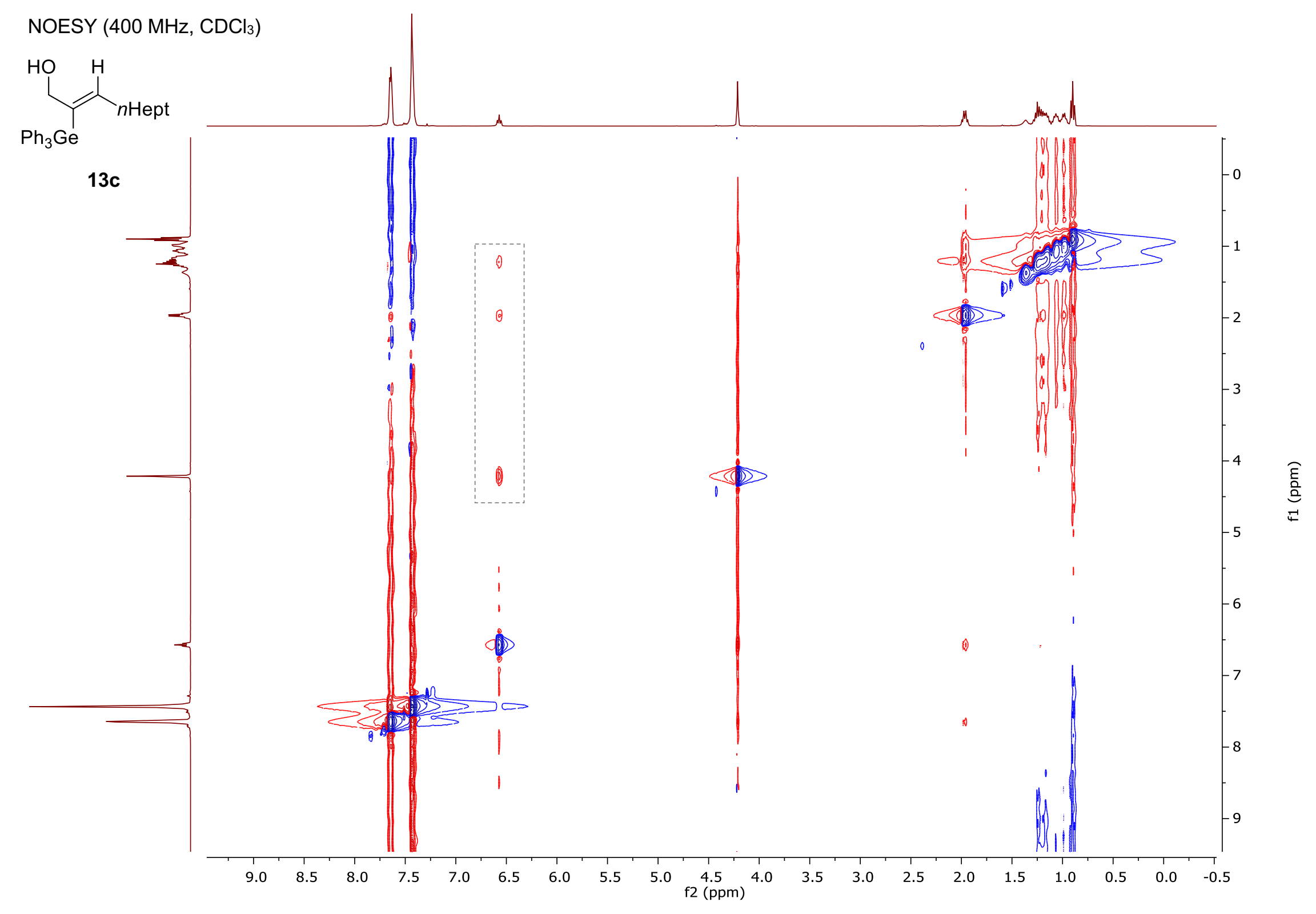




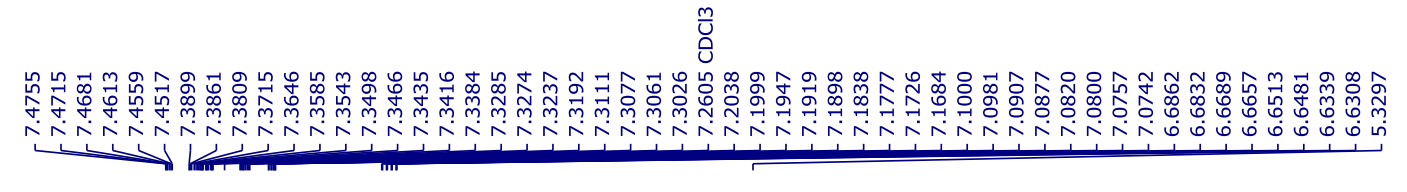

${ }^{1} \mathrm{H}$ NMR $\left(400 \mathrm{MHz}, \mathrm{CDCl}_{3}\right)$

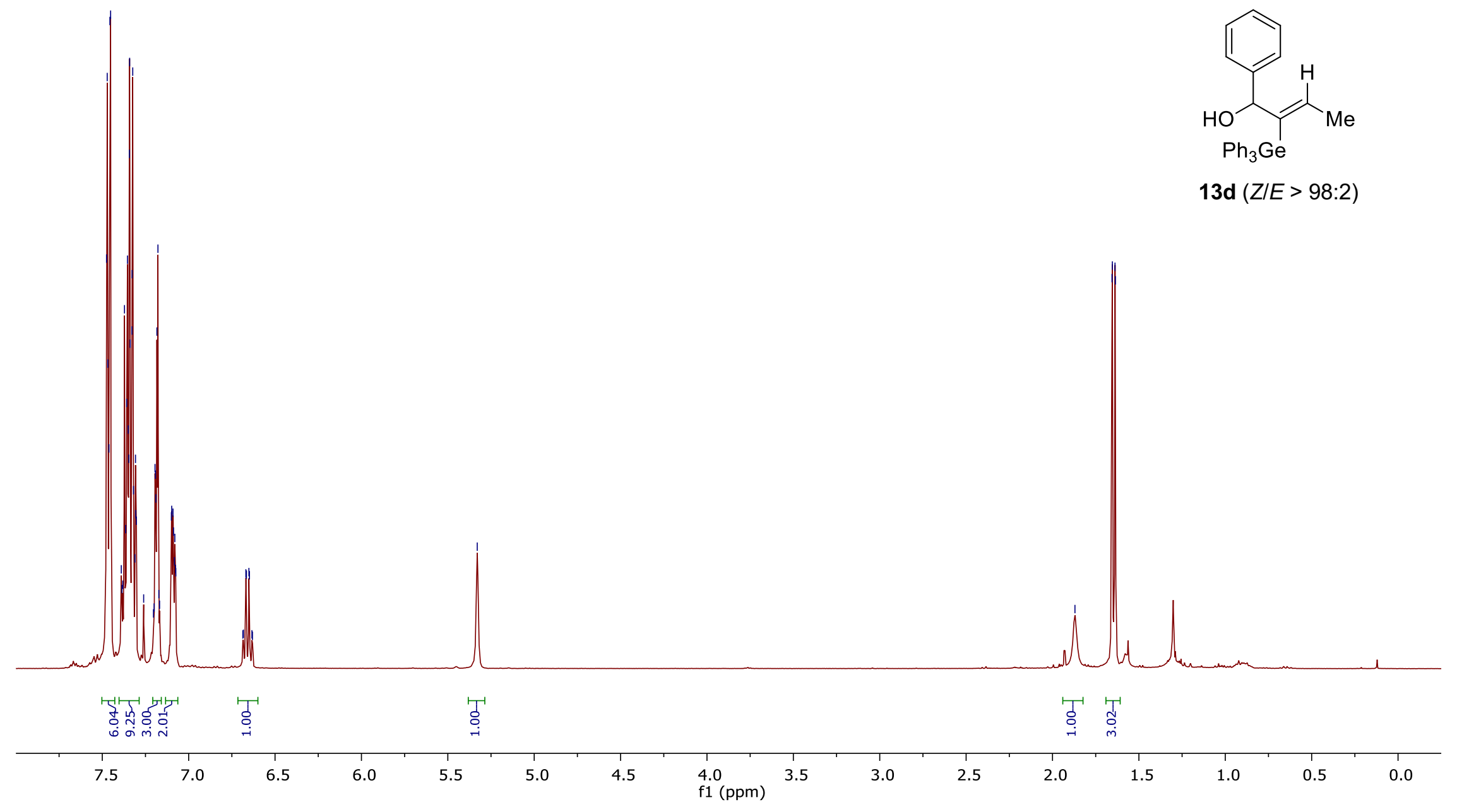




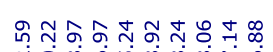

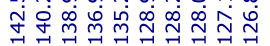

11111,

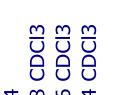

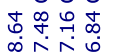

VI

${ }^{13} \mathrm{C} \mathrm{NMR}\left(100 \mathrm{MHz}, \mathrm{CDCl}_{3}\right)$

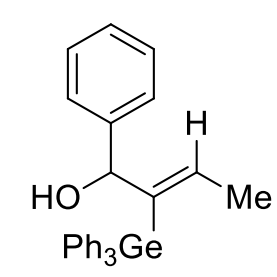

13d (Z/E > 98:2)

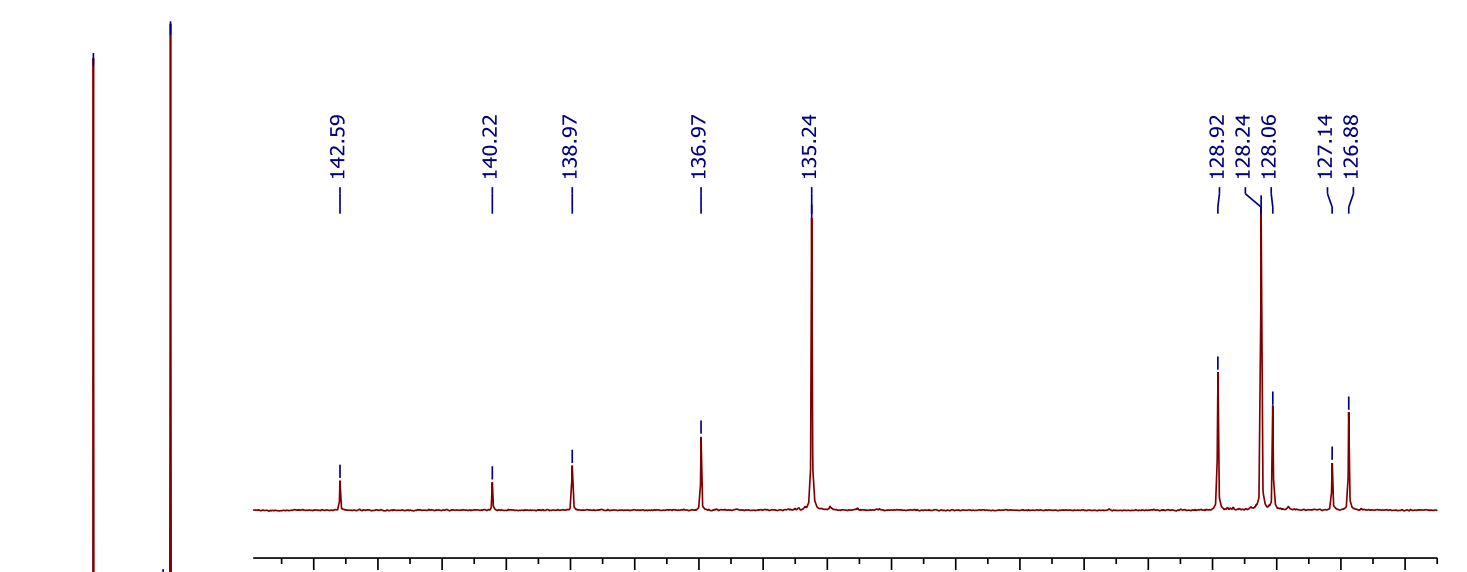

$\begin{array}{llllllllllllllllll}143 & 142 & 141 & 140 & 139 & 138 & 137 & 136 & 135 & 134 & 133 & 132 & 131 & 130 & 129 & 128 & 127 & 126\end{array}$ f1 (ppm)

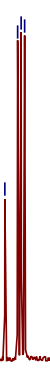

00 


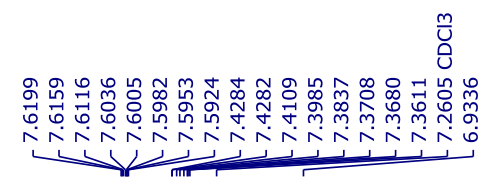

${ }^{1} \mathrm{H}$ NMR (400 MHz, $\left.\mathrm{CDCl}_{3}\right)$

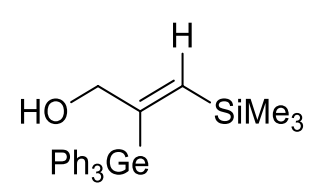

13e (Z/E > 98:2)

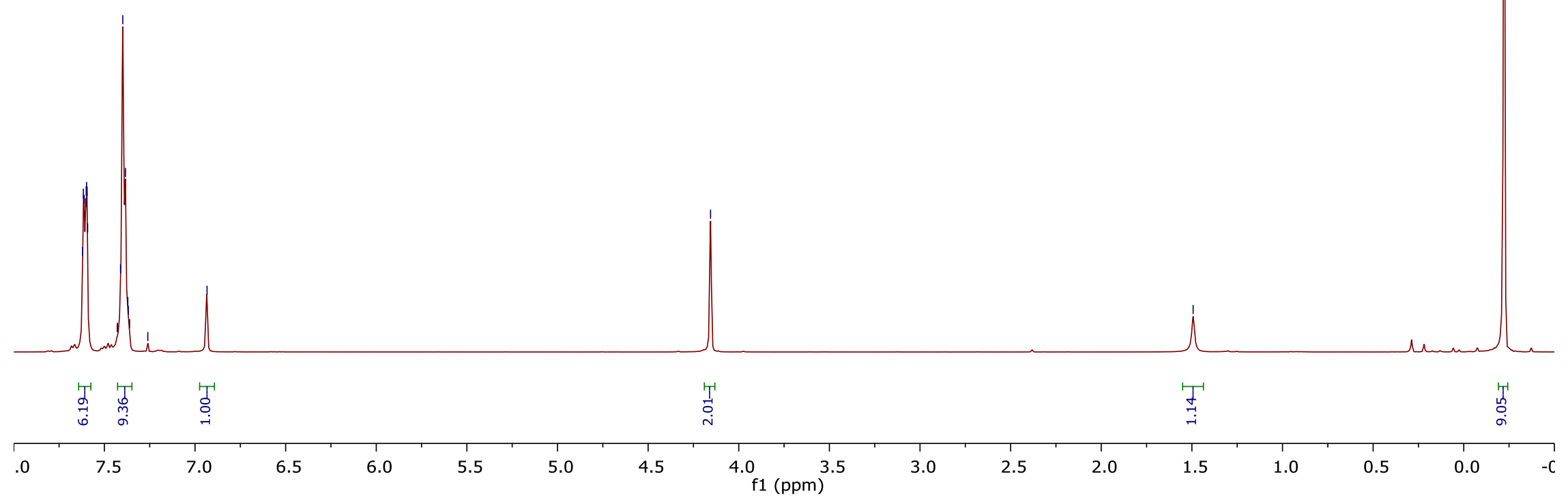




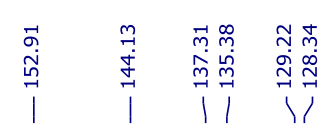

商商旁

站

र下足

${ }^{13} \mathrm{C}$ NMR $\left(100 \mathrm{MHz}, \mathrm{CDCl}_{3}\right)$

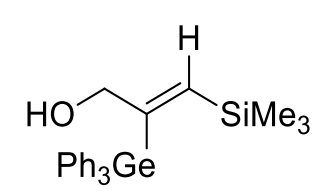

13e $(Z / E>98: 2)$

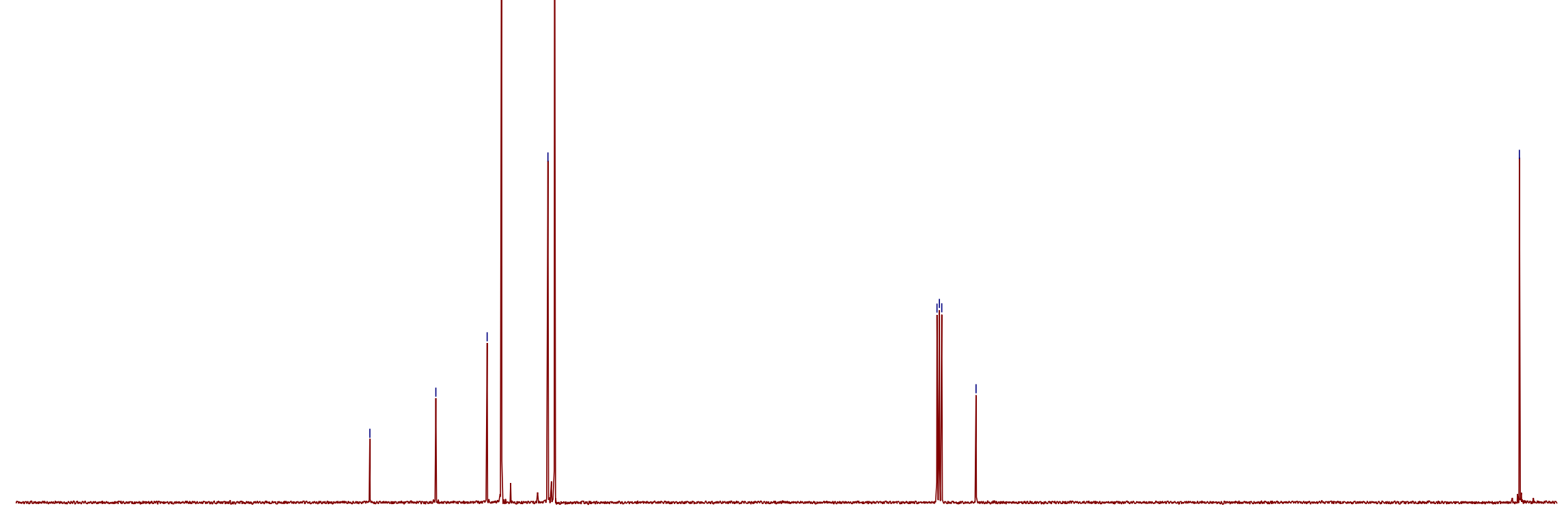




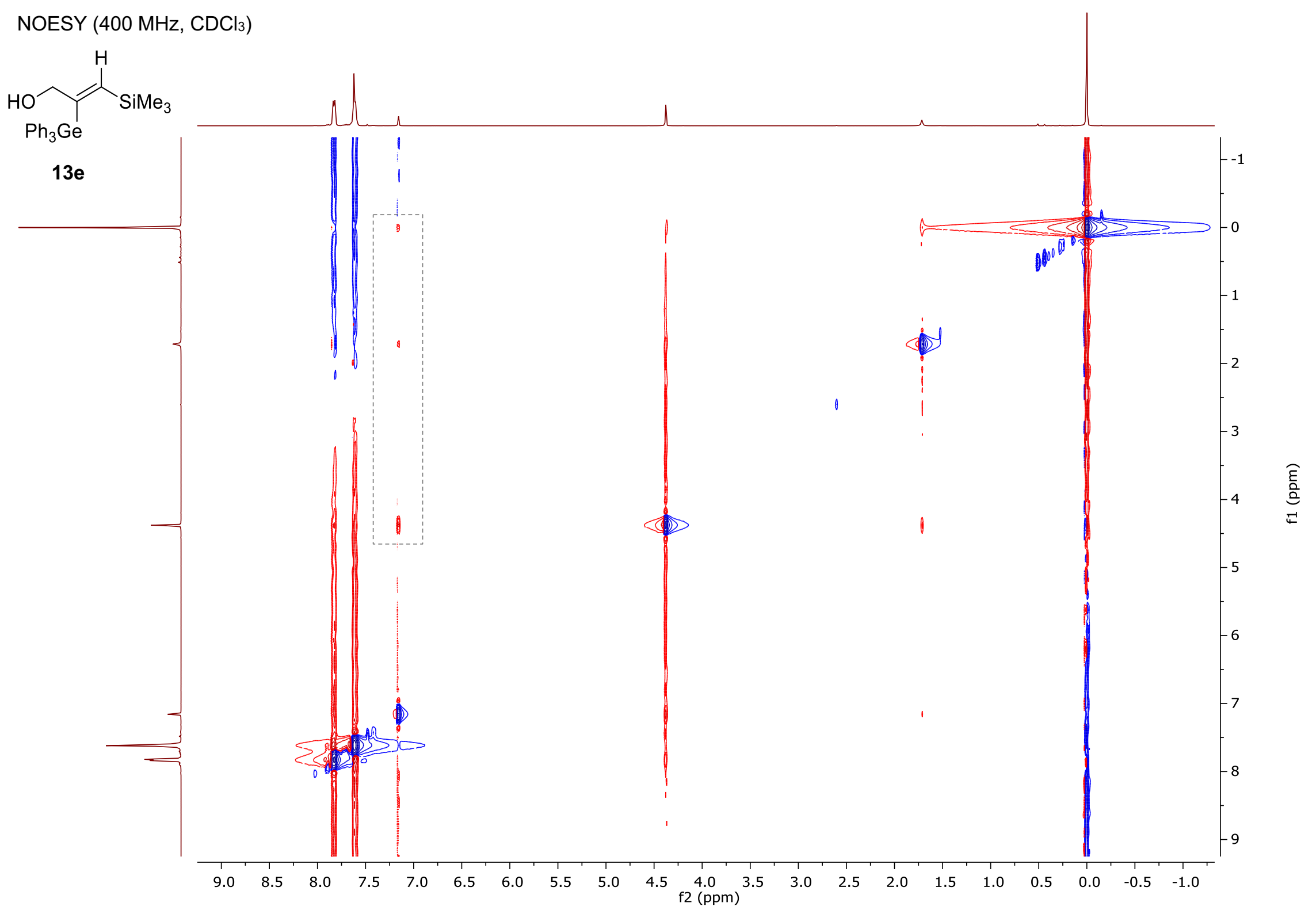




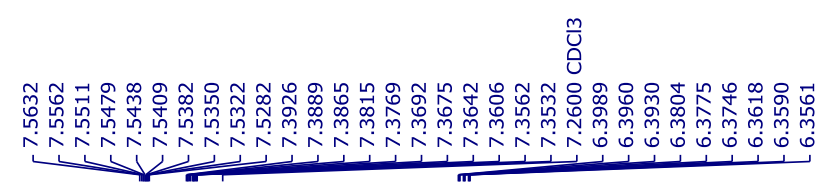

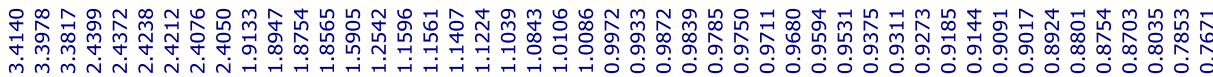

${ }^{1} \mathrm{H}$ NMR (400 MHz, $\left.\mathrm{CDCl}_{3}\right)$

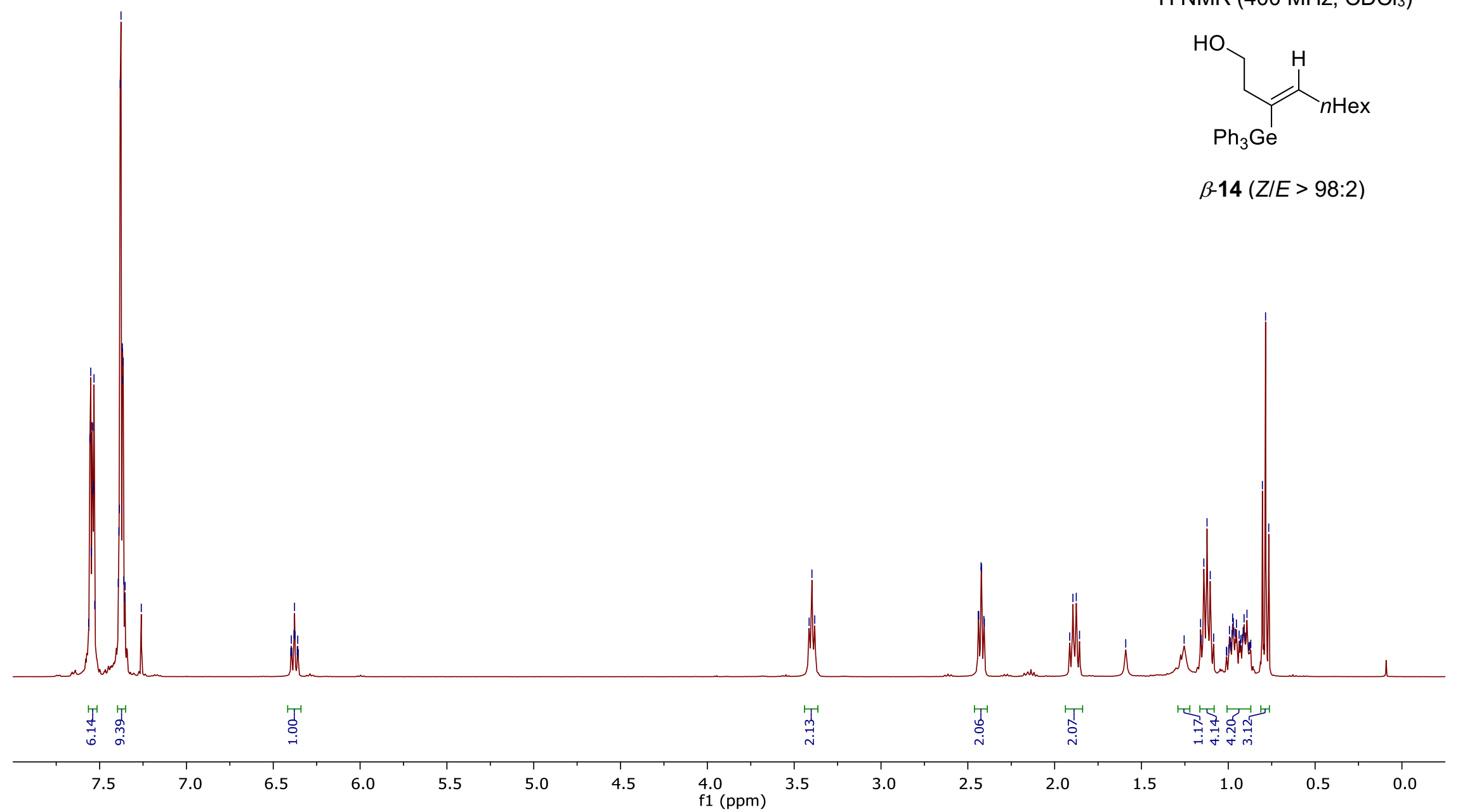


${ }^{13} \mathrm{C}$ NMR $\left(100 \mathrm{MHz}, \mathrm{CDCl}_{3}\right)$

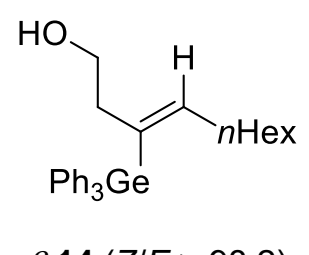

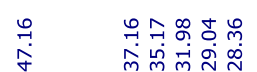

$$
\begin{aligned}
& \text { + }
\end{aligned}
$$

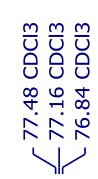

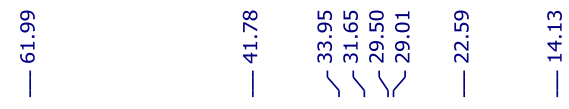

$\beta-14(Z / E>98: 2)$

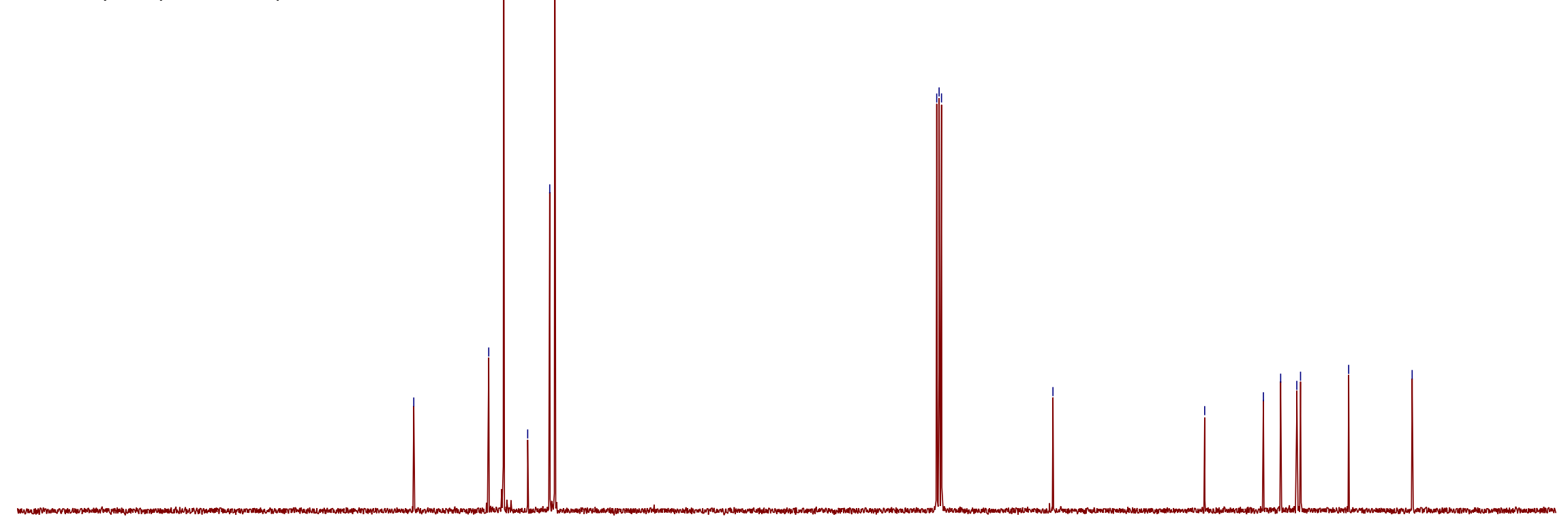




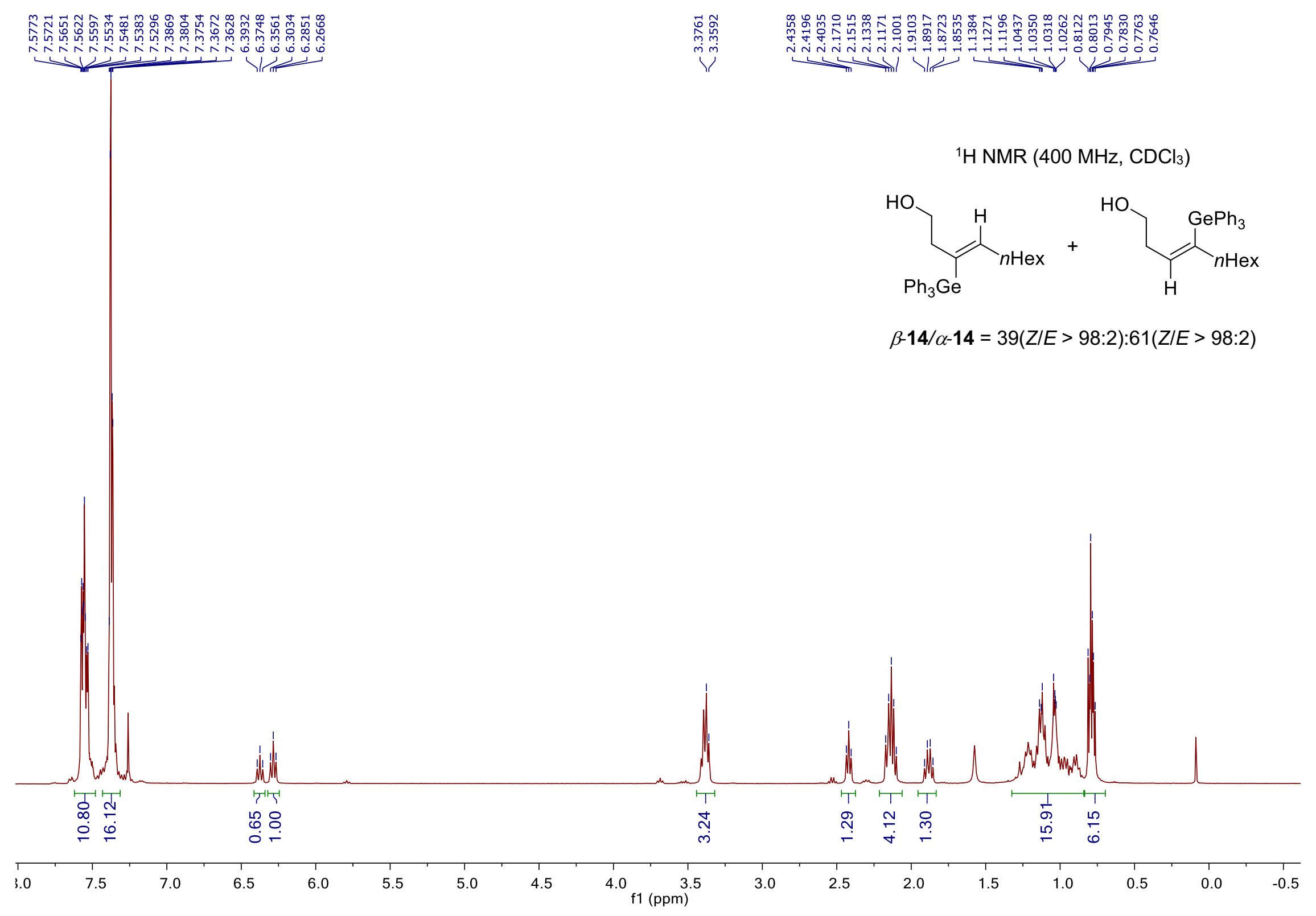




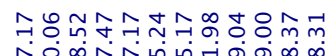

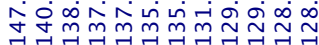

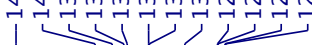

商产商

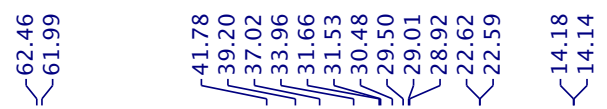

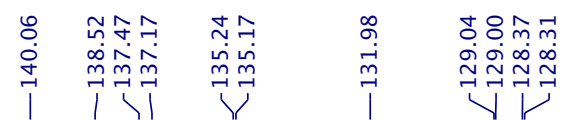

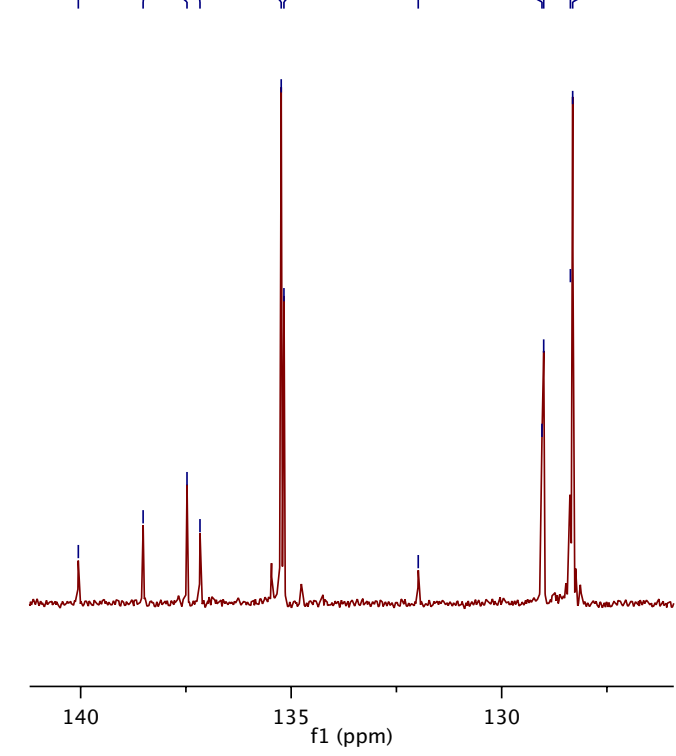

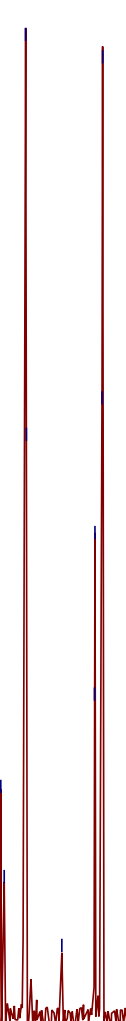

${ }^{13} \mathrm{C}$ NMR $\left(100 \mathrm{MHz}, \mathrm{CDCl}_{3}\right)$

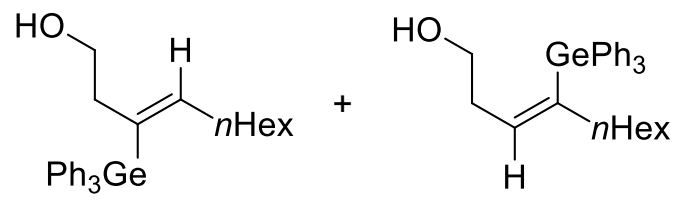

$\beta-14 / \alpha-14=39(Z / E>98: 2): 61(Z / E>98: 2)$

00

$190 \quad 180$

170

160

150

140

$130 \quad 120$

100
$\mathrm{f} 1(\mathrm{ppm})$

90

80

70

60

50

40

30 0 


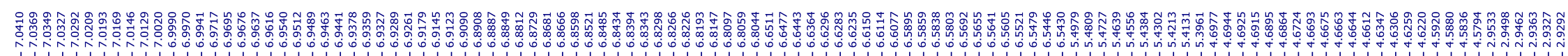

${ }^{1} \mathrm{H}$ NMR $\left(400 \mathrm{MHz}, \mathrm{CDCl}_{3}\right)$

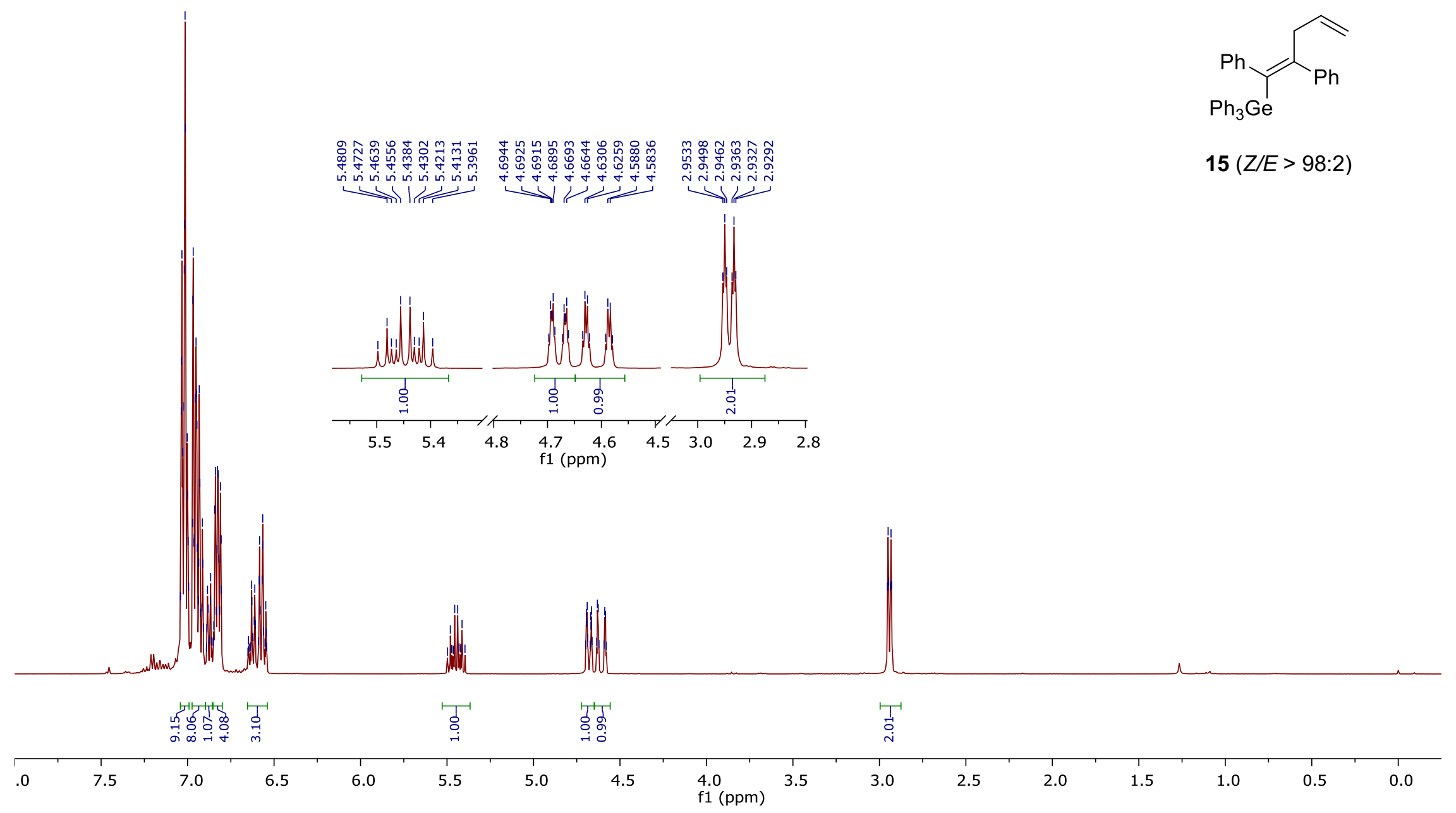



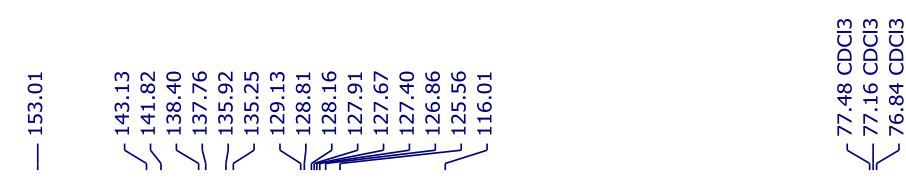

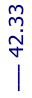

$\left.{ }^{13} \mathrm{C} \mathrm{NMR} \mathrm{(100} \mathrm{MHz,} \mathrm{CDCl}\right)$

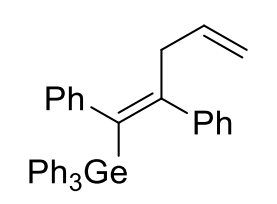

15 (Z/E > 98:2)

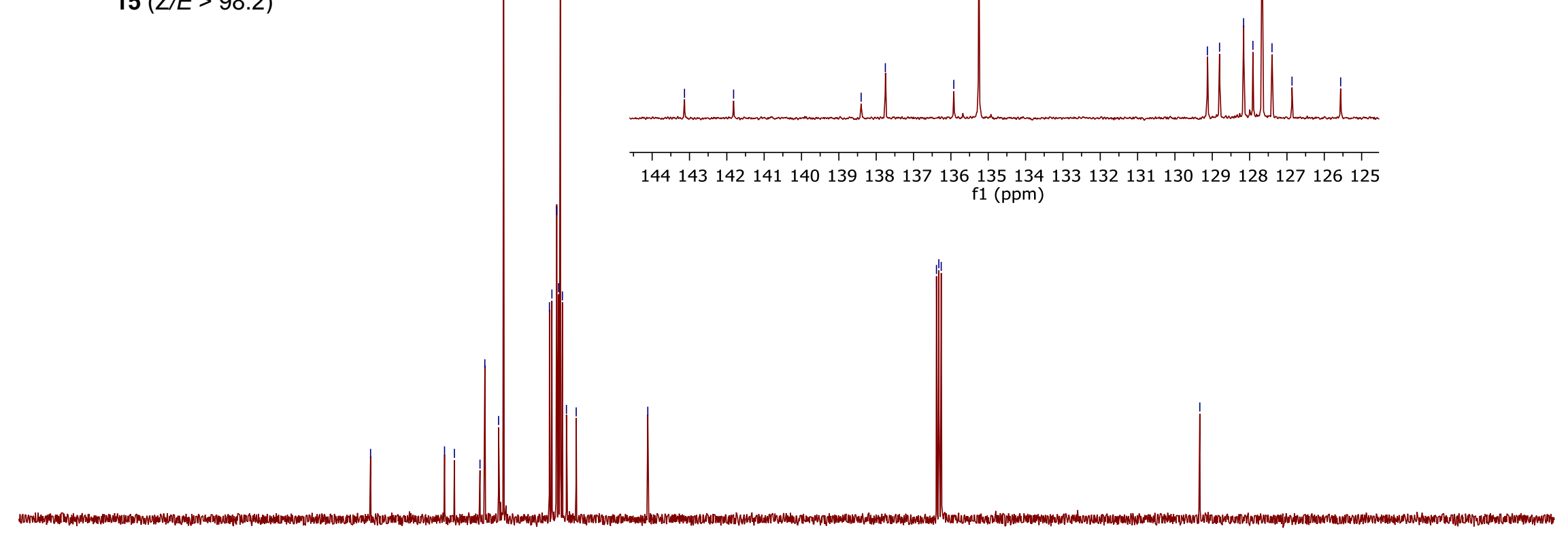




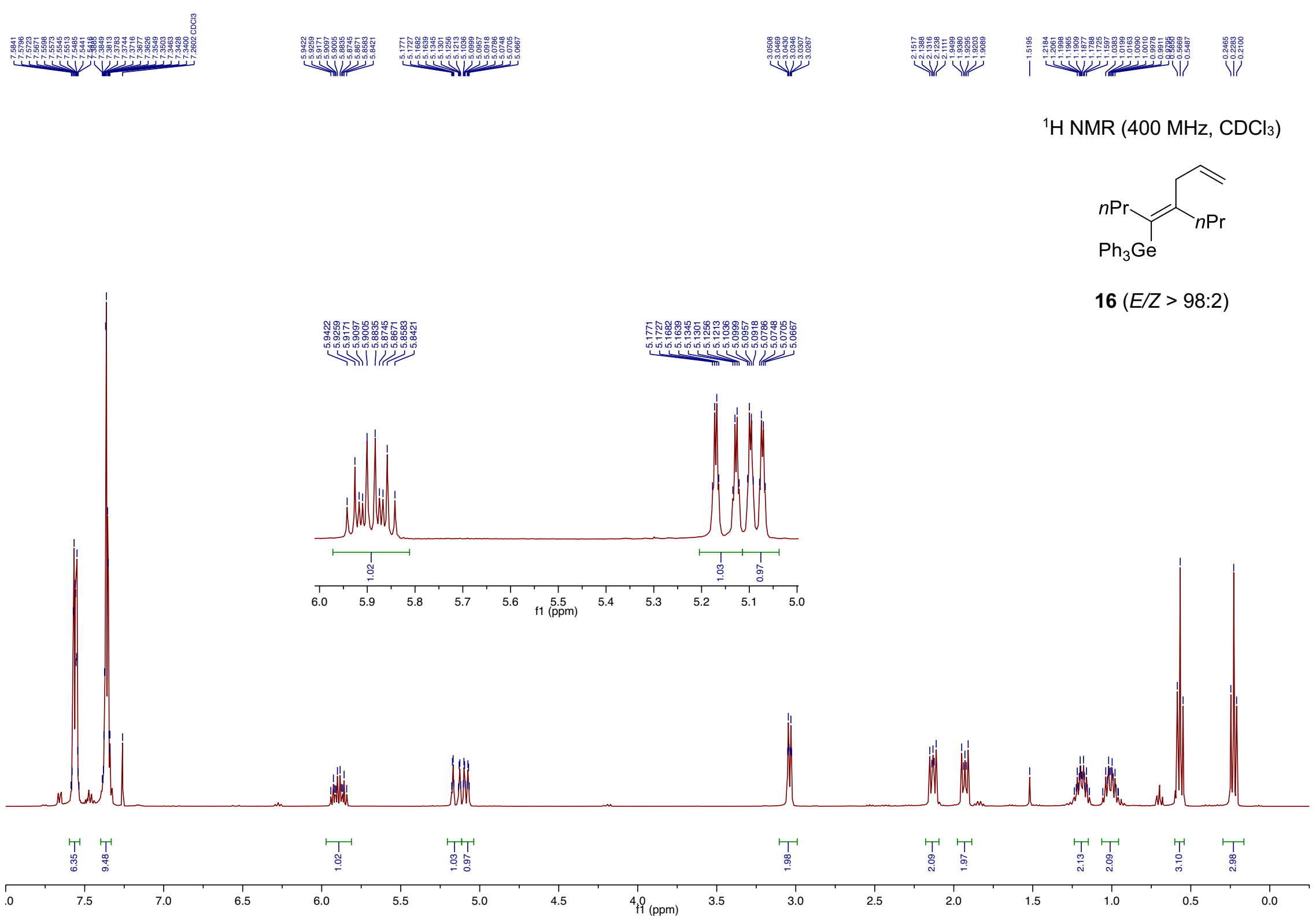




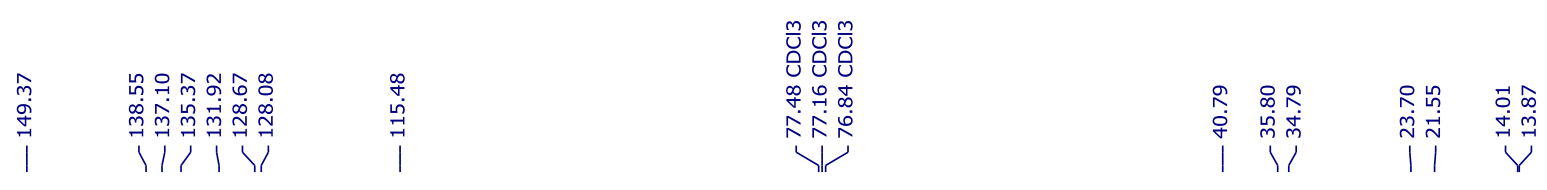

${ }^{13} \mathrm{C}$ NMR $\left(100 \mathrm{MHz}, \mathrm{CDCl}_{3}\right)$

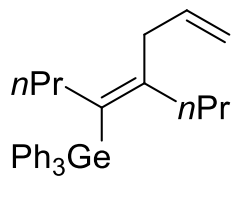

$16(E / Z>98: 2)$

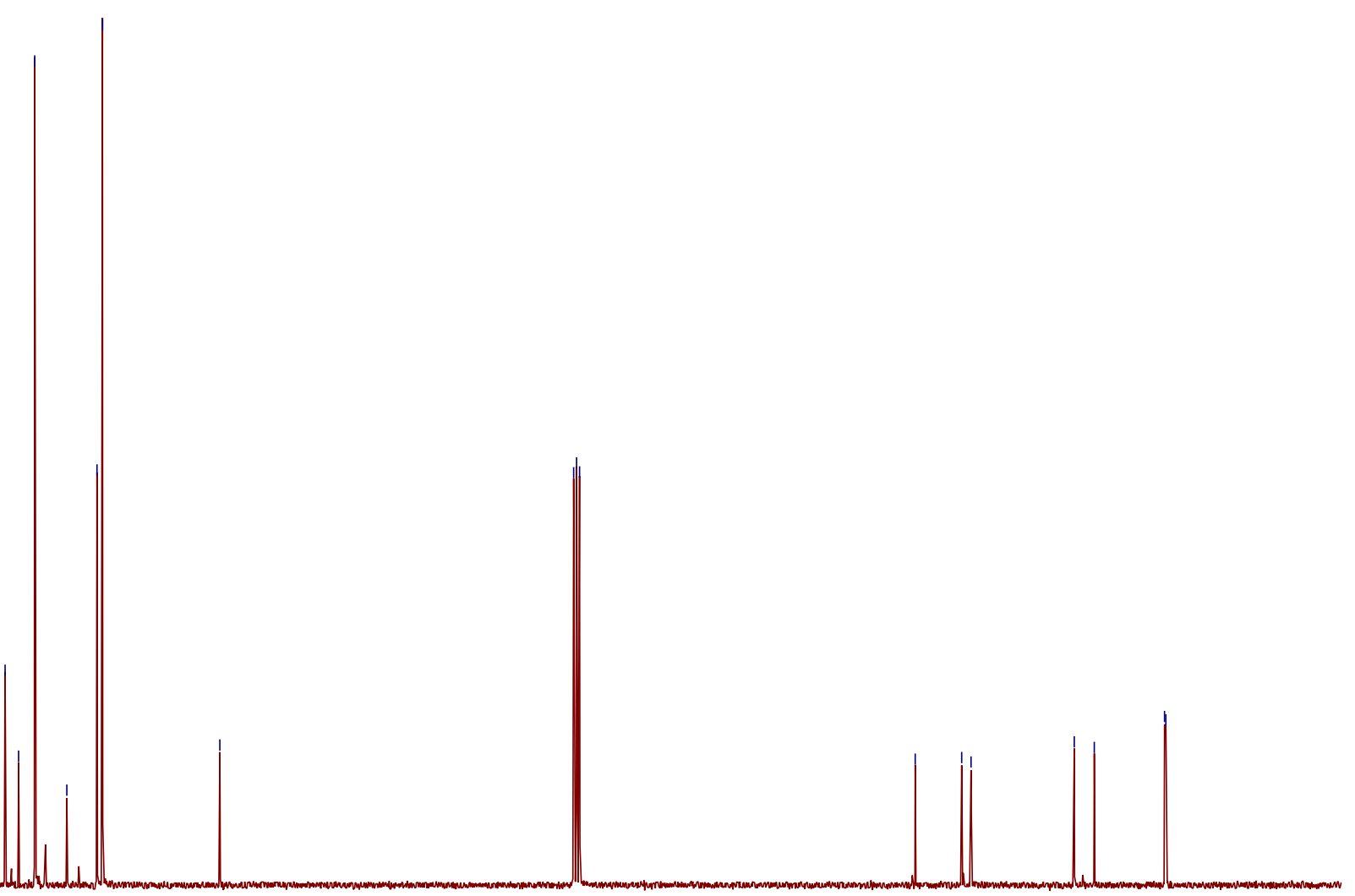




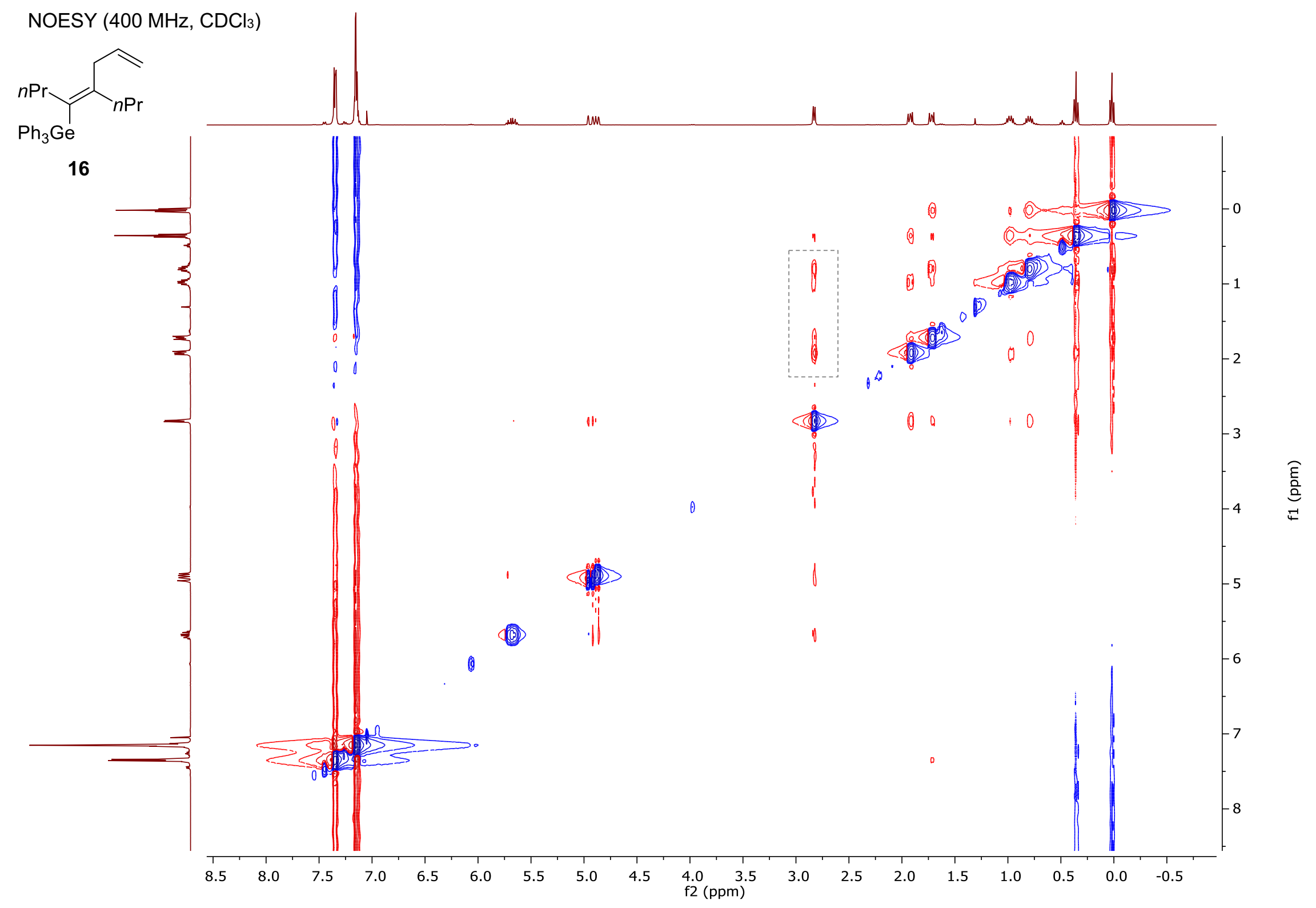




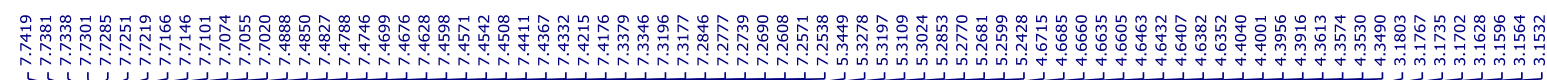

${ }^{1} \mathrm{H}$ NMR $\left(400 \mathrm{MHz}, \mathrm{CDCl}_{3}\right)$

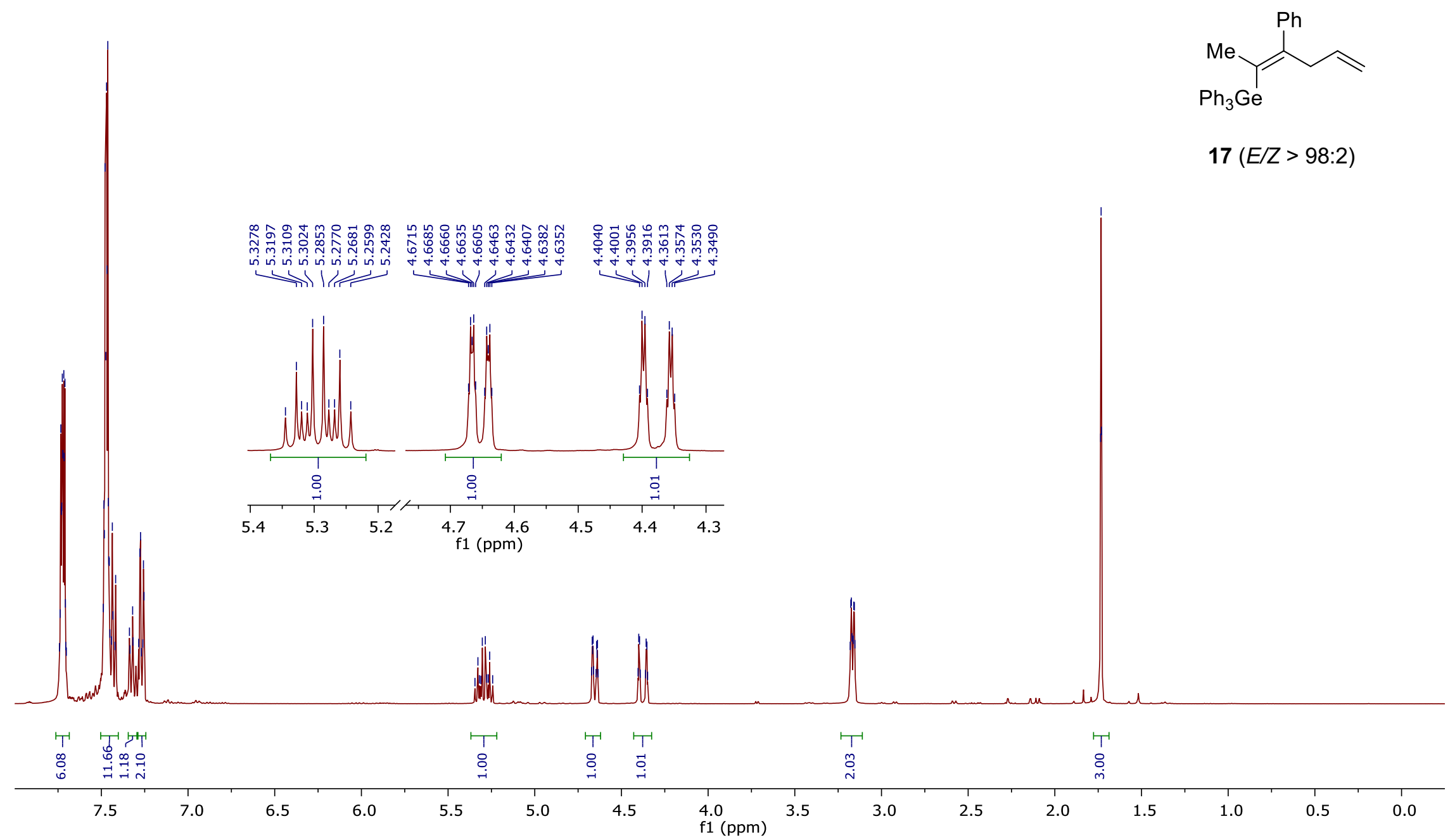




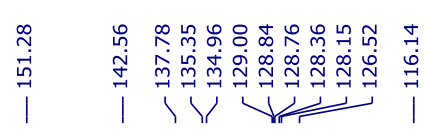

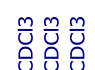

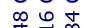

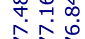

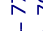

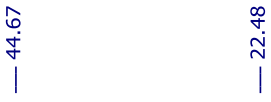

${ }^{13} \mathrm{C}$ NMR $\left(100 \mathrm{MHz}, \mathrm{CDCl}_{3}\right)$

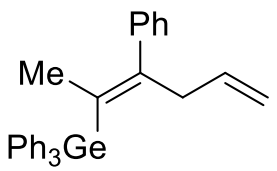

$17(E / Z>98: 2)$

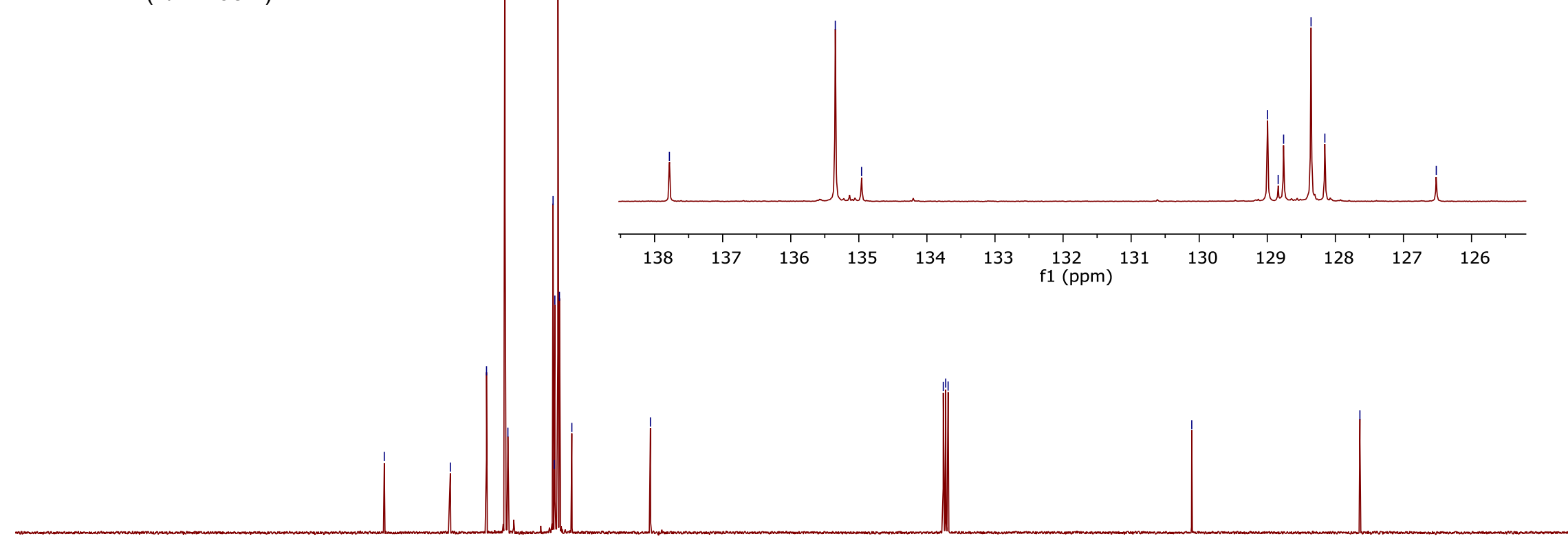




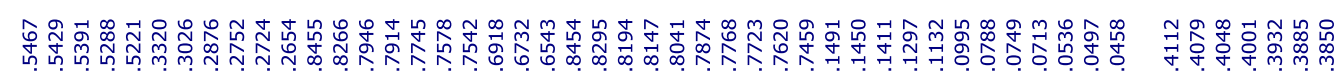

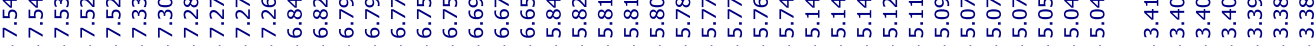

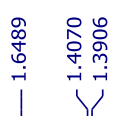

${ }^{1} \mathrm{H}$ NMR $\left(400 \mathrm{MHz}, \mathrm{CDCl}_{3}\right)$

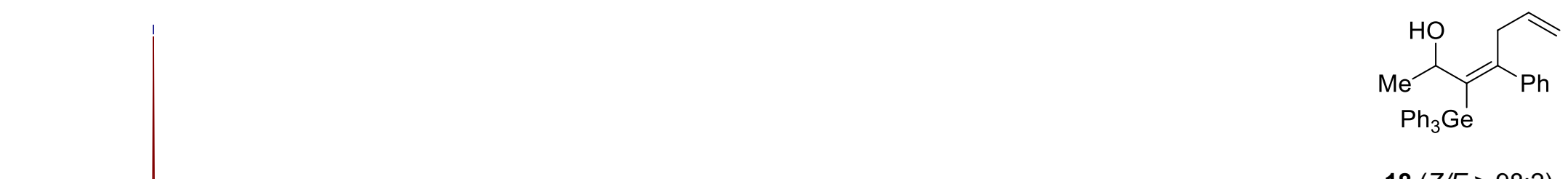

$18(Z / E>98: 2)$

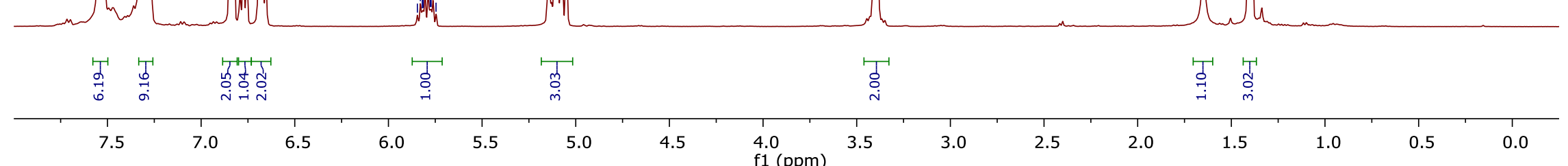



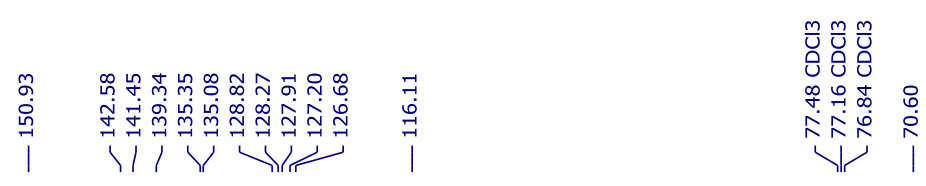

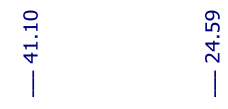

${ }^{13} \mathrm{C}$ NMR $\left(100 \mathrm{MHz}, \mathrm{CDCl}_{3}\right)$

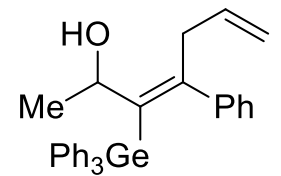

18 (Z/E > 98:2)
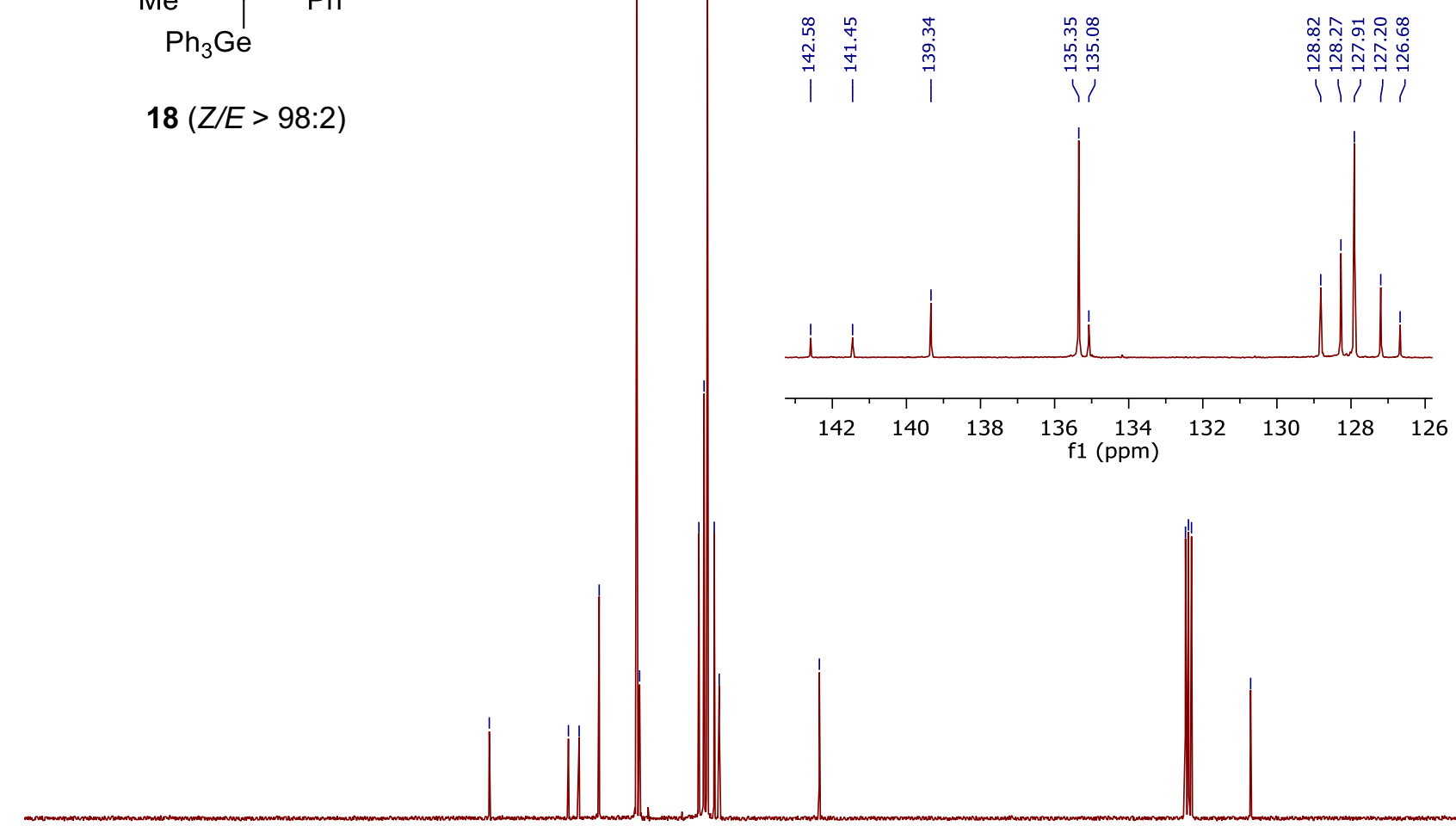

00 


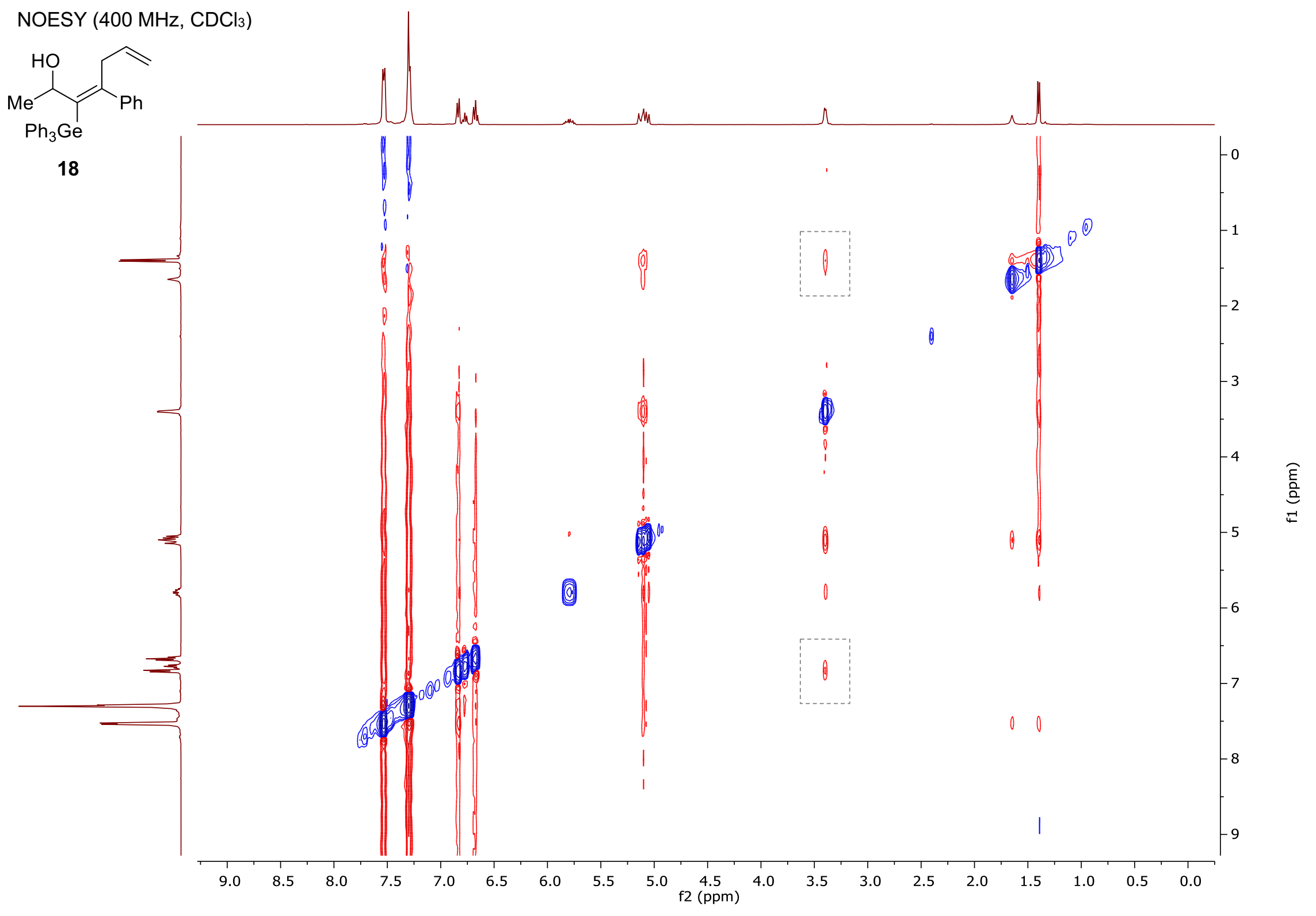


${ }^{1} \mathrm{H}$ NMR $\left(400 \mathrm{MHz}, \mathrm{CDCl}_{3}\right)$

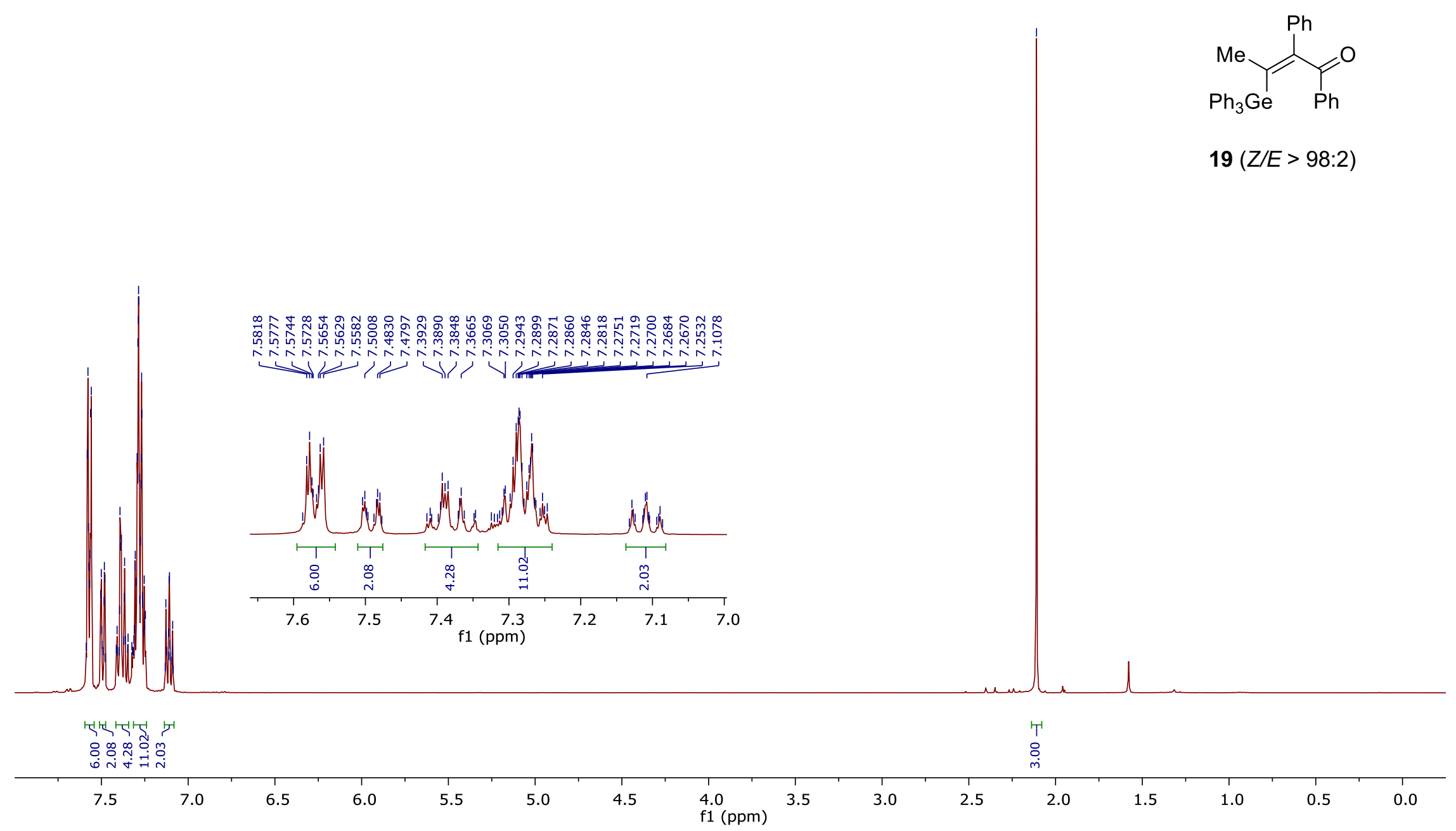




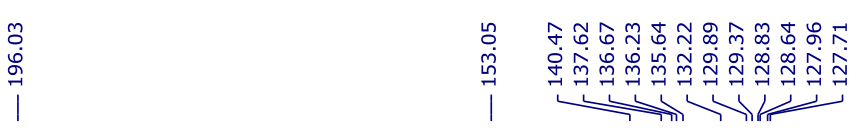

mim

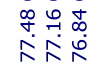

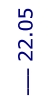

${ }^{13} \mathrm{C} \mathrm{NMR}\left(100 \mathrm{MHz}, \mathrm{CDCl}_{3}\right)$

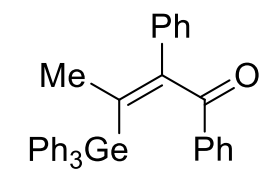

$19(Z / E>98: 2)$

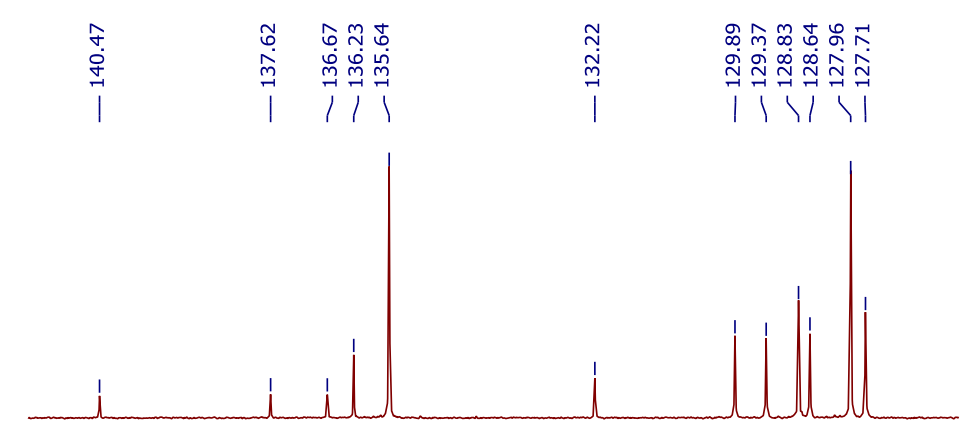

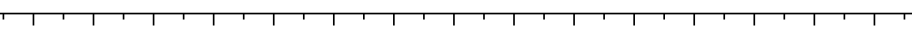

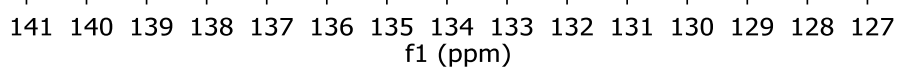

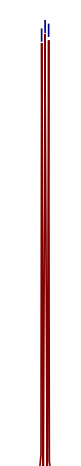

$$
11(\mathrm{ppm})
$$


$\stackrel{m}{0.00}$

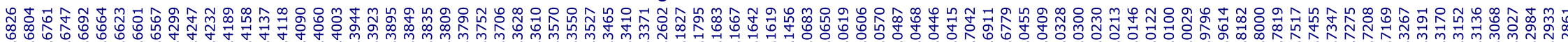

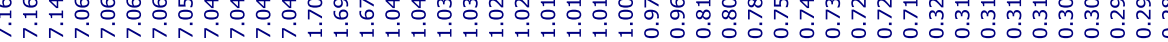

${ }^{1} \mathrm{H} \mathrm{NMR}\left(400 \mathrm{MHz}, \mathrm{CDCl}_{3}\right)$

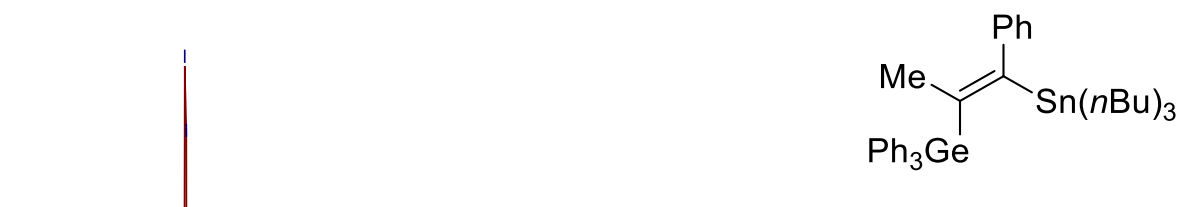

20 (Z/E > 98:2)

$\left(+9 \%\right.$ of $(n \mathrm{Bu})_{3} \mathrm{Sn}$-containing residues)
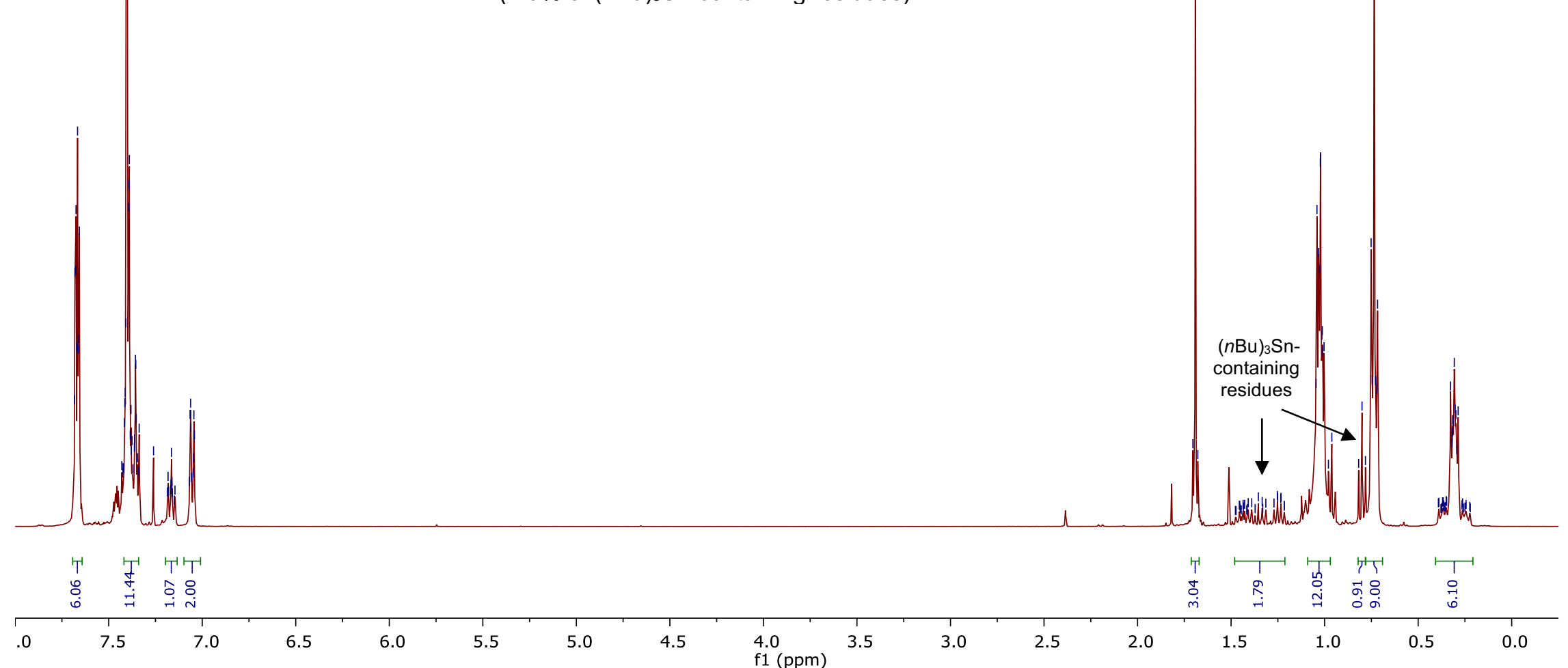

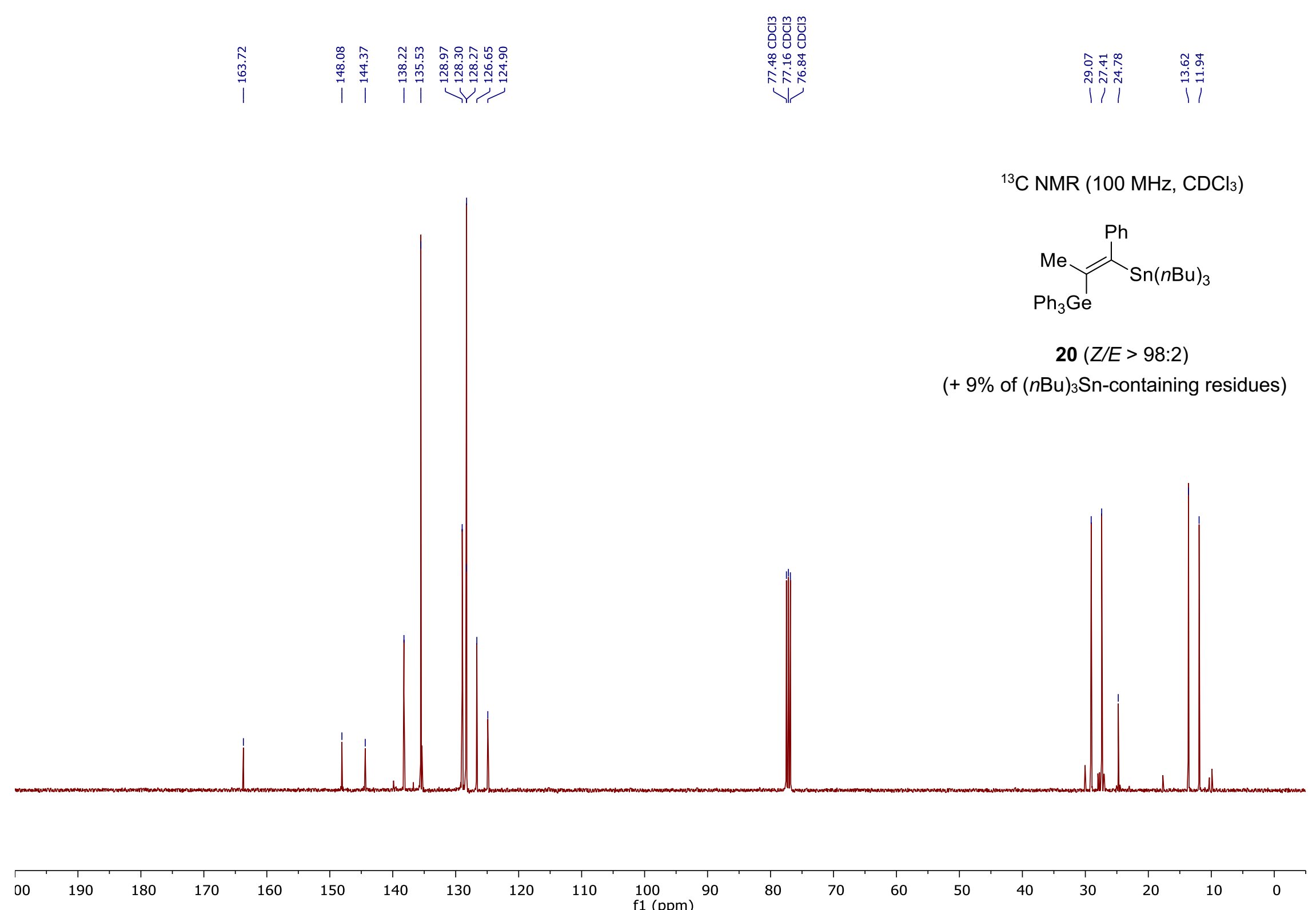


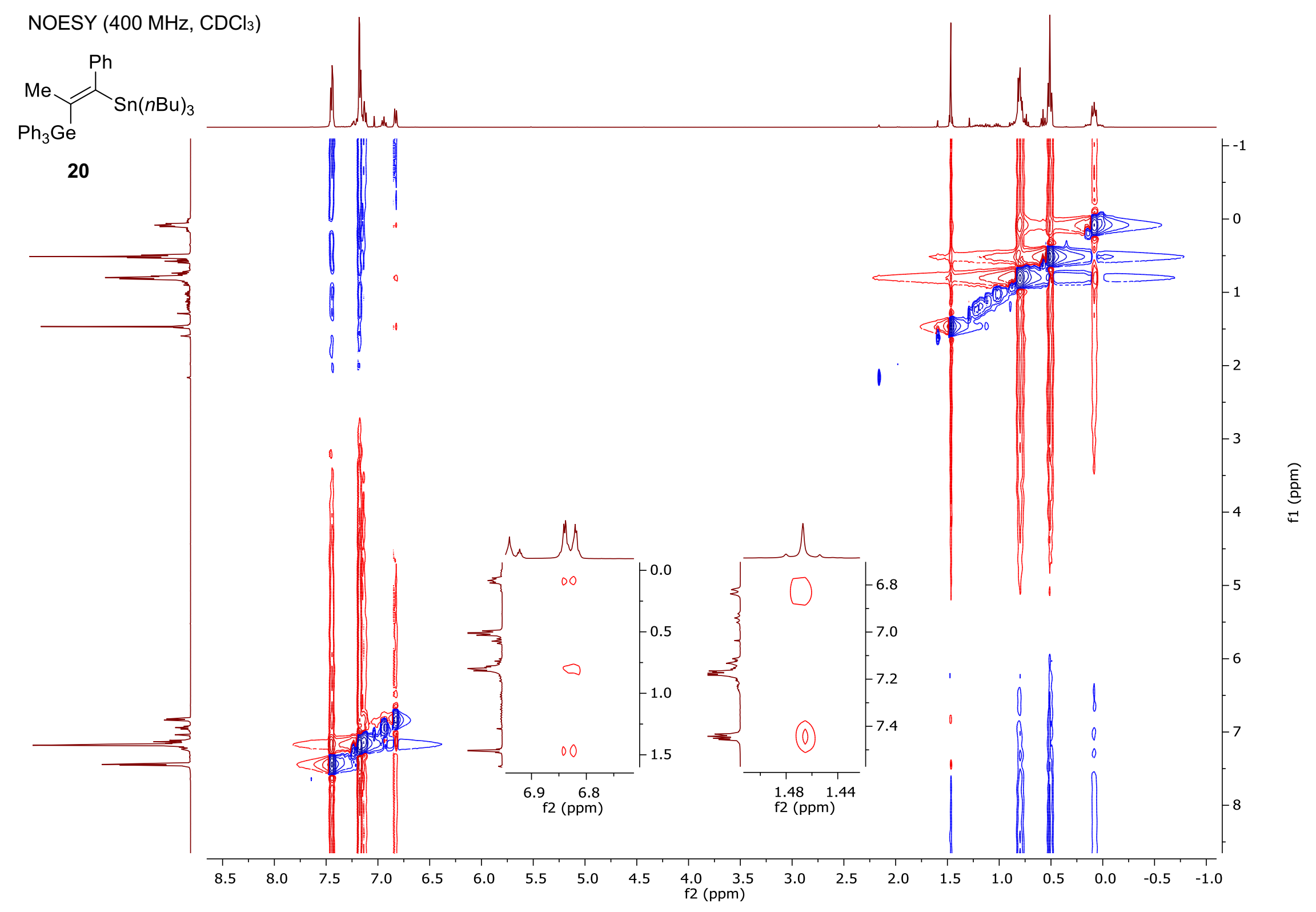




\section{II.3 X-Ray Crystal Structures of compounds 6, E-8a, Z-15, E-17}

For compound $\mathbf{6}$, crystals suitable for $\mathrm{X}$-ray analysis crystallized upon slow evaporation at room temperature of a $\mathrm{CH}_{2} \mathrm{Cl}_{2} / \mathrm{CDCl}_{3}$ solution. Compounds $8 \mathrm{a}, 15$ and 17 were dissolved completely in pentane in a $5 \mathrm{~mL}$ vial under gentle heating. $\mathrm{Et}_{2} \mathrm{O}$ was then added and the samples were allowed to cool down to room temperature and slowly evaporate until crystallization occurred providing crystals suitable for X-ray analysis. Single crystals were then selected, mounted and transferred into a cold nitrogen gas stream. Intensity data was collected with Bruker KappaAPEX2 systems using micro-source Cu-Ka or fine-focus sealed tube Mo-Ka radiation. Unitcell parameters determination, data collection strategy, integration and absorption correction were carried out with the Bruker APEX2 suite of programs. The structures were solved with SHELXT-2014 ${ }^{5}$ and refined by full-matrix least-squares methods with SHELXL-2014 ${ }^{5}$ using WinGX ${ }^{6}$ or Olex2. ${ }^{7}$ All structures were deposited at the Cambridge Crystallographic Data Centre with numbers CCDC 2077757 and CCDC 2077610-2077612 and can be obtained free of charge via www.ccdc.cam.ac.uk.

\begin{tabular}{|c|c|c|c|c|}
\hline Compound & 6 & $E-8 \mathbf{a}$ & $Z-15$ & $E-17$ \\
\hline Empirical formula & $\mathrm{C}_{44} \mathrm{H}_{44} \mathrm{Ge}_{2}$ & $\mathrm{C}_{27} \mathrm{H}_{24} \mathrm{Ge}$ & $\mathrm{C}_{35} \mathrm{H}_{30} \mathrm{Ge}$ & $\mathrm{C}_{30} \mathrm{H}_{28} \mathrm{Ge}$ \\
\hline Formula weight & 717.97 & 421.05 & 523.18 & 461.11 \\
\hline Crystal system & Triclinic & Monoclinic & Triclinic & Monoclinic \\
\hline Space group & $P-1$ & $\mathrm{P} 22_{1}$ & $P-1$ & $\mathrm{P} 2{ }_{1} / \mathrm{n}$ \\
\hline \multirow{6}{*}{ Unit cell dimensions } & $a=11.1866(7) \AA$ & $a=7.5482(3) \AA$ & $a=9.3468(6) \AA$ & $a=11.6542(2) \AA$ \\
\hline & $\mathrm{b}=12.8216(7) \AA$ & $b=11.5246(4) \AA$ & $b=10.2995(7) \AA$ & $b=9.7060(2) \AA$ \\
\hline & $c=13.6305(8) \AA$ & $c=12.3986(5) \AA$ & $c=16.0371(11) \AA$ & $c=20.9033(3) \AA$ \\
\hline & $\alpha=69.793(2)^{\circ}$ & $\alpha=90^{\circ}$ & $\alpha=102.950(4)^{\circ}$ & $\alpha=90^{\circ}$ \\
\hline & $\beta=78.159(2)^{\circ}$ & $\beta=98.078(2)^{\circ}$ & $\beta=90.213(5)^{\circ}$ & $\beta=94.4370(10)^{\circ}$ \\
\hline & $\gamma=80.010(2)^{\circ}$ & $\nu=90^{\circ}$ & $\gamma=112.772(4)^{\circ}$ & $\nu=90^{\circ}$ \\
\hline Volume & $1784.42(18) \AA^{3}$ & $1067.85(7) \AA^{3}$ & $1380.26(17) \AA^{3}$ & $2357.40(7) \AA^{3}$ \\
\hline Z & 2 & 2 & 2 & 4 \\
\hline Crystal description & colourless needle & colourless prism & colourless prism & colourless fragment \\
\hline Crystal size & $0.34 \times 0.06 \times 0.06 \mathrm{~mm}^{3}$ & $0.5 \times 0.2 \times 0.05 \mathrm{~mm}^{3}$ & $0.12 \times 0.09 \times 0.03 \mathrm{~mm}^{3}$ & $0.5 \times 0.4 \times 0.3 \mathrm{~mm}^{3}$ \\
\hline Density (calculated) & $1.336 \mathrm{~g} . \mathrm{cm}^{-3}$ & $1.309 \mathrm{~g} \cdot \mathrm{cm}^{-3}$ & $1.259 \mathrm{~g} . \mathrm{cm}^{-3}$ & $1.299 \mathrm{~g} . \mathrm{cm}^{-3}$ \\
\hline
\end{tabular}

\footnotetext{
${ }^{5}$ Sheldrick, G. M. Crystal Structure Refinement with SHELXL. Acta Cryst. C 2015, 71, 3-8.

${ }^{6}$ Farrugia, L. J. WinGX and ORTEP for Windows: an Update. J. Appl. Cryst. 2012, 45, 849-854.

${ }^{7}$ Dolomanov, O. V.; Bourhis, L. J.; Gildea, R. J.; Howard, J. A. K.; Puschmann, H. OLEX2: a Complete Structure Solution, Refinement and Analysis Program. J. Appl. Cryst. 2009, 42, 339-341.
} 
X-Ray structure of compound 6 (CCDC 2077757) - Image showing 50\% probability ellipsoids

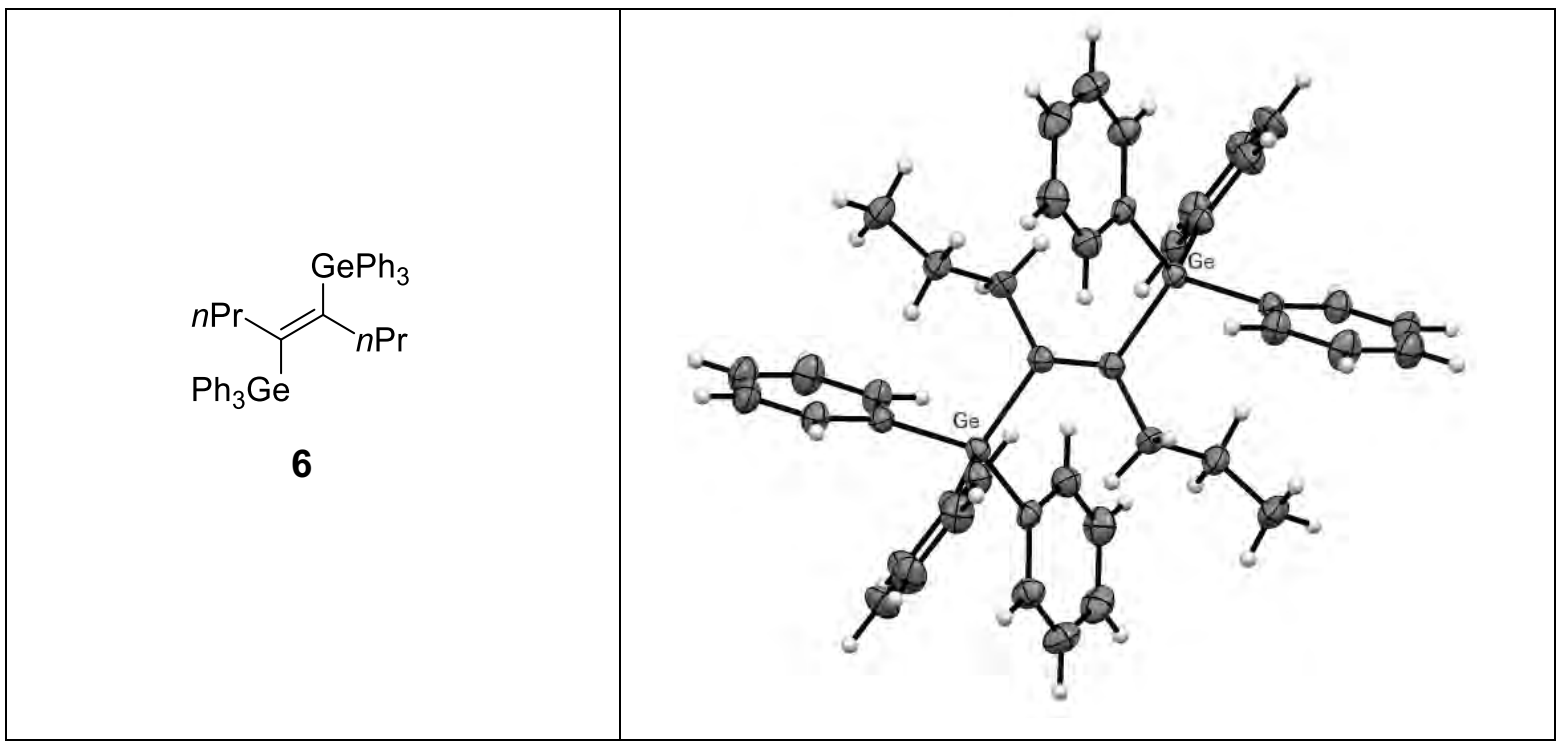

X-Ray structure of compound E-8a (CCDC 2077610) - Image showing 50\% probability ellipsoids

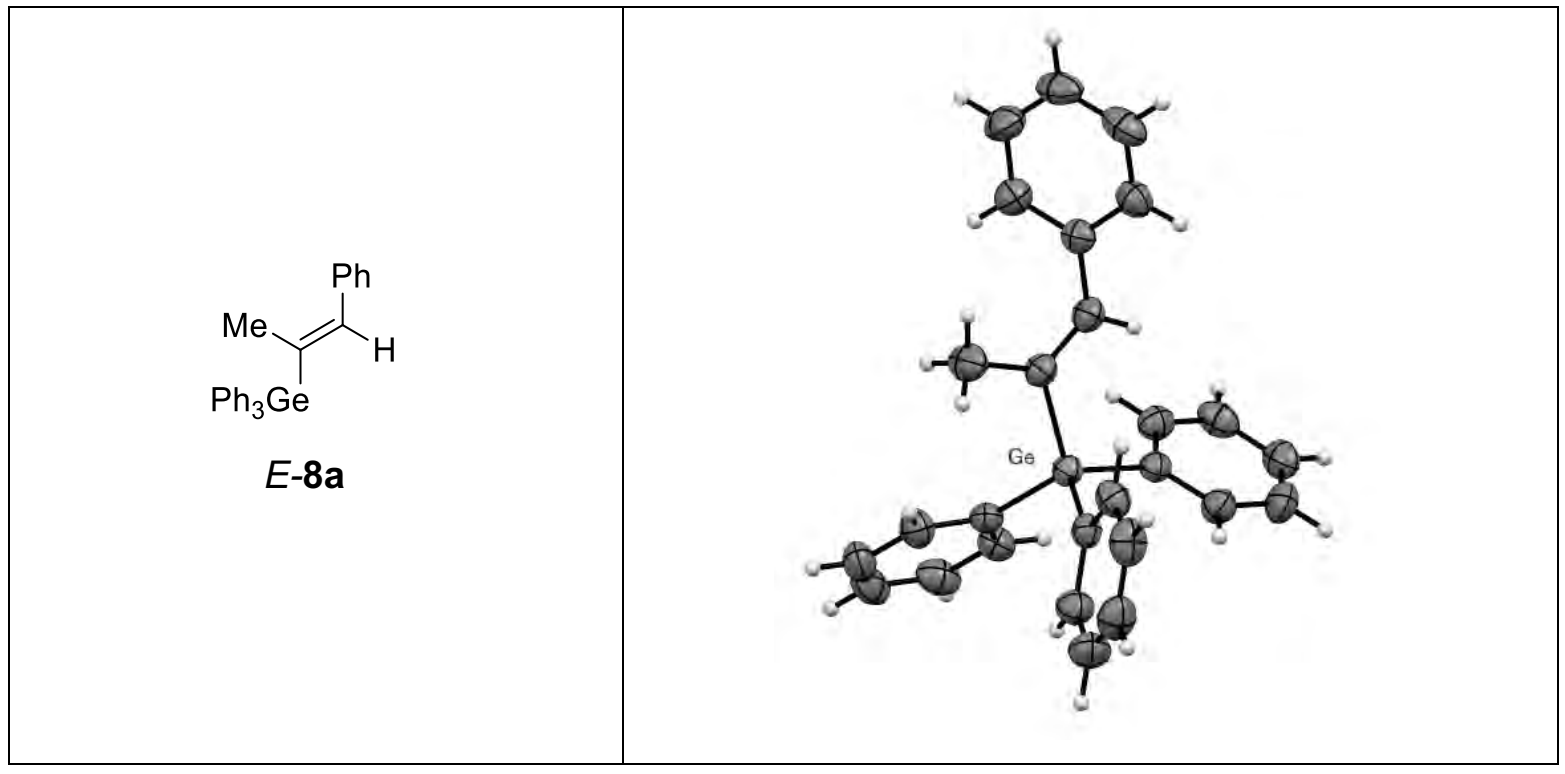


X-Ray structure of compound Z-15 (CCDC 2077611) - Image showing 50\% probability ellipsoids

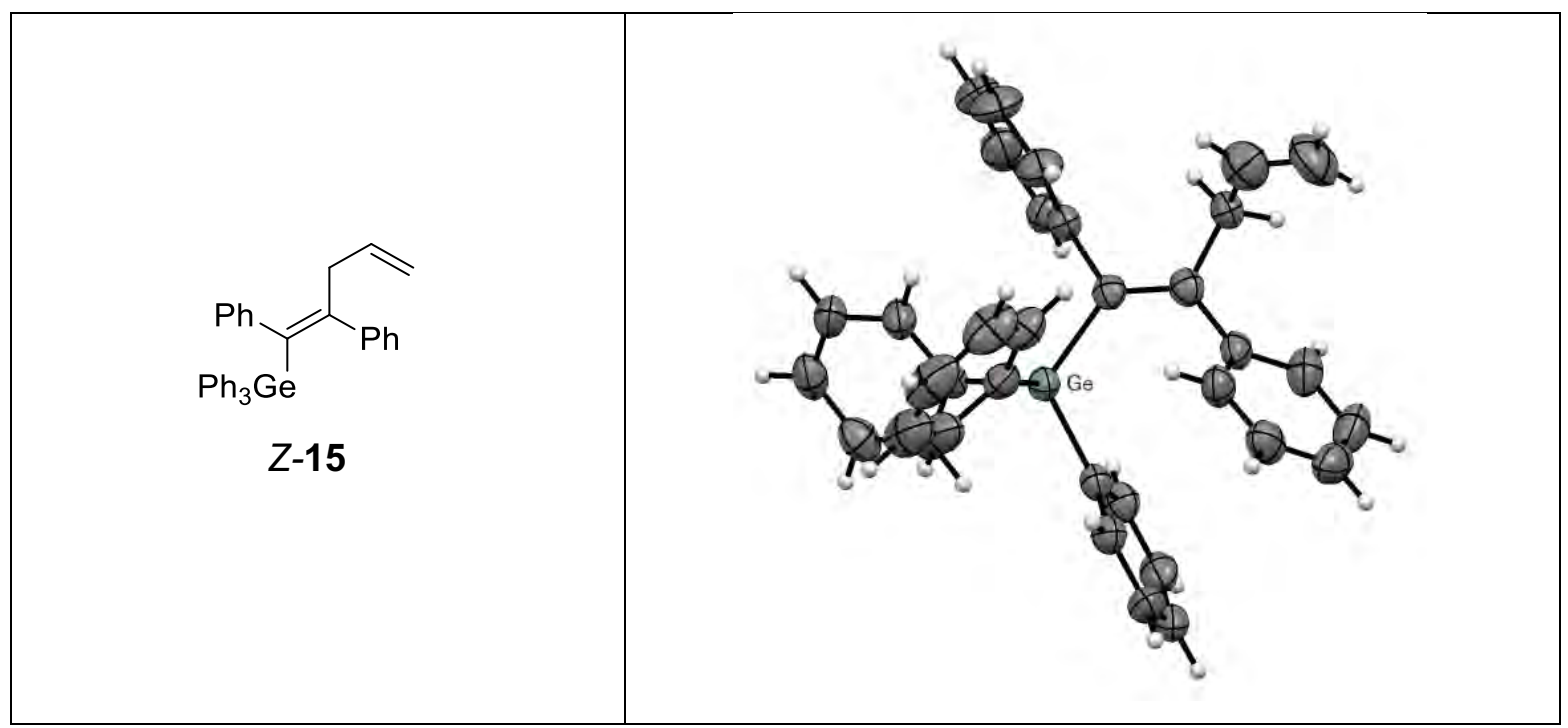

X-Ray structure of compound E-17 (CCDC 2077612) - Image showing 50\% probability ellipsoids

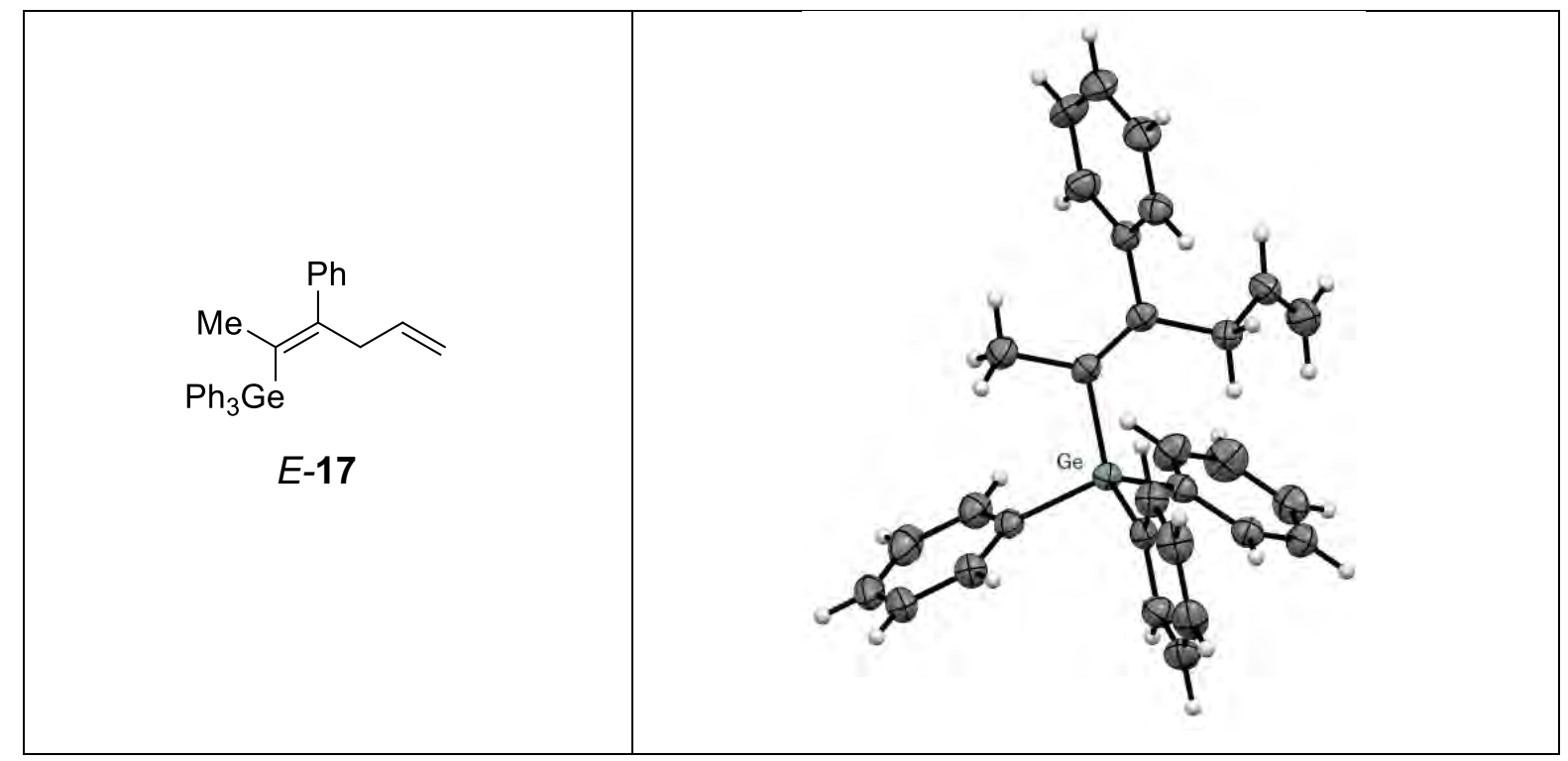

